Florida International University FIU Digital Commons

\title{
Proton Form Factor Puzzle and the CEBAF Large Acceptance Spectrometer(CLAS) Two-Photon Exchange Experiment
}

Dipak Rimal

drima001@fiu.edu

DOI: $10.25148 /$ etd.FI14040856

Follow this and additional works at: https://digitalcommons.fiu.edu/etd

Part of the Nuclear Commons

\section{Recommended Citation}

Rimal, Dipak, "Proton Form Factor Puzzle and the CEBAF Large Acceptance Spectrometer(CLAS) Two-Photon Exchange Experiment" (2014). FIU Electronic Theses and Dissertations. 1211.

https://digitalcommons.fiu.edu/etd/1211 


\section{FLORIDA INTERNATIONAL UNIVERSITY \\ Miami, Florida}

\section{PROTON FORM FACTOR PUZZLE AND THE CEBAF LARGE ACCEPTANCE SPECTROMETER (CLAS) TWO-PHOTON EXCHANGE EXPERIMENT}

A dissertation submitted in partial fulfillment of the

requirements for the degree of
DOCTOR OF PHILOSOPHY
in
PHYSICS
by

Dipak Rimal

2014 


\section{To: Dean Kenneth G. Furton}

College of Arts and Sciences

This dissertation, written by Dipak Rimal, and entitled Proton Form Factor Puzzle and the CEBAF Large Acceptance Spectrometer (CLAS) Two-Photon Exchange Experiment, having been approved in respect to style and intellectual content, is referred to you for judgment.

We have read this dissertation and recommend that it be approved.

$\begin{array}{r}\hline \text { Werner U. Boeglin } \\ \hline \text { Oren V. Maxwell } \\ \hline \text { Cem Karayalcin } \\ \hline \text { Brian A. Raue, Major Professor }\end{array}$

Date of Defense: March 27, 2014

The dissertation of Dipak Rimal is approved.

Dean Kenneth G. Furton College of Arts and Sciences

Dean Lakshmi N. Reddi University Graduate School

Florida International University, 2014 
(C) Copyright 2014 by Dipak Rimal All rights reserved. 


\section{DEDICATION}

This dissertation is dedicated to my entire family. This work would not have been possible without their tremendous support, encouragement, patience, understanding, and their unconditional love. 


\section{ACKNOWLEDGMENTS}

For this accomplishment, I would like to express my sincere gratitude to my family, friends, and professors for their continuous support and encouragement.

At first, I would like to express my heart-felt gratitude to my excellent advisor Prof. Brian Raue for his continuous support, intellectual guidance, expert suggestions, and most of all, the trust he put in me. This work would not have been possible without his support, guidance, and trust. He continuously guided me towards academic excellence and taught me to "think" before knocking on his door for an easy answer. I would like to thank him for being an excellent mentor and a great teacher throughout my graduate career. I wish to thank Prof. Raue along with the Department of Energy for funding this work.

I would like to express my deepest gratitude to Dr. Puneet Khetarpal for his support and guidance through various stages of this work. I learned the basics of programming in $\mathrm{C}++$ and ROOT along with the nuclear physics data analysis techniques from him. I would like to thank Dr. Khetarpal from the core of my heart for his support and willingness to help during difficult times.

I would also like to thank members of my committee including Profs. Werner U. Boeglin, Oren V. Maxwell, and Cem Karayalcin for their passionate support at several stages of writing this dissertation. I would like to thank all the physics professors for their support and guidance throughout my graduate career.

This work would not be possible without the tremendous effort put forth by the members of the CLAS TPE Collaboration, especially Prof. Lawrence Weinstein, Dr. Robert Bennett, and fellow graduate student Dasuni Adikaram. Though only my

name appears in the title page, this dissertation is a product of the collaboration with these individuals. I would like to express my sincere gratitude to each of these 
individuals. I would also like to thank Drs. John Arrington and Will Brooks for providing their expert opinion and input during data analysis. I would like to thank the entire CLAS Collaboration as well as Jefferson Lab scientists and engineering staff for their support and effort, which made this work a success.

I would like to thank my office-mates at CP184 for their support and encouragement. Special thank goes to Eric Pooser for patiently reading the first draft of this dissertation to improve my writing. Many thanks to Hari, Jason, and Will for physics and programming discussions.

I am indebted to my late father Hem Chandra Rimal for the sacrifice he made to ensure that I had an excellent education. I am deeply grateful to my mother Mina Rimal, brother Niraj, and sister Anjana for their unconditional love and support for my education. I owe more to them than I can express. I want to express my love and heart-felt gratitude to my wife Abha for her support and sacrifice. Finally, a lot of love to my little princess Adhista. 


\title{
ABSTRACT OF THE DISSERTATION \\ PROTON FORM FACTOR PUZZLE AND THE CEBAF LARGE ACCEPTANCE SPECTROMETER (CLAS) TWO-PHOTON EXCHANGE EXPERIMENT
}

by

\author{
Dipak Rimal \\ Florida International University, 2014 \\ Miami, Florida \\ Professor Brian A. Raue, Major Professor
}

The electromagnetic form factors are the most fundamental observables that encode information about the internal structure of the nucleon. The electric $\left(G_{E}\right)$ and the magnetic $\left(G_{M}\right)$ form factors contain information about the spatial distribution of the charge and magnetization inside the nucleon. A significant discrepancy exists between the Rosenbluth and the polarization transfer measurements of the electromagnetic form factors of the proton. One possible explanation for the discrepancy is the contributions of two-photon exchange (TPE) effects. Theoretical calculations estimating the magnitude of the TPE effect are highly model dependent, and limited experimental evidence for such effects exists. Experimentally, the TPE effect can be measured by comparing the ratio of positron-proton elastic scattering cross section to that of the electron-proton $\left(R=\frac{\sigma\left(e^{+} p\right)}{\sigma\left(e^{-} p\right)}\right)$. The ratio $R$ was measured over a wide range of kinematics, utilizing a $5.6 \mathrm{GeV}$ primary electron beam produced by the Continuous Electron Beam Accelerator Facility (CEBAF) at Jefferson Lab. This dissertation explored dependence of $R$ on kinematic variables such as squared four-momentum transfer $\left(Q^{2}\right)$ and the virtual photon polarization parameter $(\varepsilon)$.

A mixed electron-positron beam was produced from the primary electron beam in experimental Hall B. The mixed beam was scattered from a liquid hydrogen $\left(\mathrm{LH}_{2}\right)$ target. Both the scattered lepton and the recoil proton were detected by the CEBAF 
Large Acceptance Spectrometer (CLAS). The elastic events were then identified by using elastic scattering kinematics.

This work extracted the $Q^{2}$ dependence of $R$ at high $\varepsilon(\varepsilon>0.8)$ and the $\varepsilon$ dependence of $R$ at $\left\langle Q^{2}\right\rangle \approx 0.85 \mathrm{GeV}^{2}$. In these kinematics, our data confirm the validity of the hadronic calculations of the TPE effect by Blunden, Melnitchouk, and Tjon. This hadronic TPE effect, with additional corrections contributed by higher excitations of the intermediate state nucleon, largely reconciles the Rosenbluth and the polarization transfer measurements of the electromagnetic form factors. 


\section{TABLE OF CONTENTS}

CHAPTER

PAGE

1 Introduction 1

1.1 Overview . . . . . . . . . . . . . . . . . . . . . . . . . . . 1

2 An Overview of the Proton Form Factors 6

2.1 Elastic Electromagnetic Form Factors . . . . . . . . . . . . . . . . . . 6

2.1 .1 Kinematic Variables . . . . . . . . . . . . . . . . 6

2.1 .2 Nucleon Form Factors: Basics . . . . . . . . . . . . . . . . . . 10

2.1.3 Elastic Electromagnetic Form Factors Formalism . . . . . . . 11

2.2 Measurements of Electromagnetic Form Factors in the Born Approxi-

mation ........................... 14

2.2 .1 Rosenbluth Separation Method . . . . . . . . . . . . . . 15

2.2 .2 Polarization Transfer (PT) Method . . . . . . . . . . . . . . . 17

2.3 Proton Form Factor Puzzle . . . . . . . . . . . . . . . . . . . . . . . . 18

2.4 Possible Resolution of the Puzzle: Two Photon Exchange Effects . . . 21

2.5 Theoretical Calculations of TPE . . . . . . . . . . . . . . . 23

2.5.1 Guichon and Vanderhaeghen Calculation . . . . . . . . . . . 23

2.5 .2 BMT Calculation . . . . . . . . . . . . . . . . . 26

2.5.3 Generalized Parton Distribution (GPD) Model . . . . . . . . . 30

2.5.4 Summary of the Theoretical Calculations of TPE . . . . . . . 32

$2.6 \quad$ Experimental Measurement of Two-Photon Exchange Effect . . . . . 33

$2.7 \quad$ Existing Data for the Cross Section Ratio . . . . . . . . . . . . . . 35

$\begin{array}{lll}3 & \text { Experimental Apparatus } & 37\end{array}$

3.1 Overview . . . . . . . . . . . . . . . . . . . 37

3.2 CEBAF . . . . . . . . . . . . . . . . . . . 37

3.3 Hall B and CLAS Detector . . . . . . . . . . . . . . . . . . . . . . . . . . . . . . . . . . . . . . 38

3.3 .1 Torus Magnet . . . . . . . . . . . . . . . . . . . . . . . . . . . . 39

3.3.2 Mini-Torus Magnet . . . . . . . . . . . . . . . . . . . . . . . . . . . . . . . . . 42

3.3.3 Drift Chamber (DC) . . . . . . . . . . . . . . . . . . . . . . . . 42

3.3.4 Time of Flight (TOF) Counters . . . . . . . . . . . . . . . 45

3.3.5 Electromagnetic Calorimeter (EC) . . . . . . . . . . . . . . . 46

3.4 TPE Beamline Components . . . . . . . . . . . . . . . . . . . . . . . . . . . . . . . .

3.4.1 Chicane . . . . . . . . . . . . . . . . . . . . . . . . . . 49

3.4 .2 Sparse Fiber Monitor . . . . . . . . . . . . . . . . . . . . . . 50

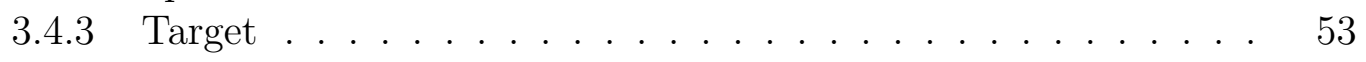

3.4.4 Downstream Beam Profile System . . . . . . . . . . . . . . . . 55

3.5 Background Control . . . . . . . . . . . . . . . . . . . 57

3.6 Trigger and Data Acquisition System . . . . . . . . . . . . . . . . . 59

3.7 Data Collection . . . . . . . . . . . . . . . . 61 
3.8 Event Reconstruction . . . . . . . . . . . . . . . . . . . . . . . . . . . . . 63

3.9 Detector Calibrations . . . . . . . . . . . . . . . . . . . . . . . . 64

3.9 .1 Drift Chamber Calibrations . . . . . . . . . . . . . . . 64

3.10 Kinematic Corrections . . . . . . . . . . . . . . . . . 66

3.10 .1 Energy Loss Corrections . . . . . . . . . . . . . . . . . . 68

3.10 .2 Momentum Corrections. . . . . . . . . . . . . . . . . . 69

4 Data Analysis 76

$4.1 \quad$ Elastic Event Selection . . . . . . . . . . . . . . . . . . . 76

4.1 .1 Elastic Kinematic Cuts . . . . . . . . . . . . . . . . . . . 80

4.1 .2 Beam Energy Distribution . . . . . . . . . . . . . . . . . 90

4.2 Dead Detector Corrections . . . . . . . . . . . . . . . . . . . . . . . . 91

4.2 .1 Inefficient Detector Removal . . . . . . . . . . . . . . . . . . . 91

4.2 .2 Swimming Corrections . . . . . . . . . . . . . . . . . . 92

4.3 Kinematic Coverage and Bin Selection . . . . . . . . . . . . . . 93

4.4 Background Subtraction . . . . . . . . . . . . . . . . . . . . . . . . . . . 95

4.5 The Cross Section Ratio $\ldots \ldots \ldots$. . . . . . . . . . . . . . . . 97

$4.6 \quad$ Results from the Analysis of TPECal Data . . . . . . . . . . . . . . 101

5 Results and Discussions 103

5.1 Radiative Corrections . . . . . . . . . . . . . . . . . . . . . . 103

5.2 Systematic Uncertainties . . . . . . . . . . . . . . . . . . . . 107

5.2 .1 Effects of Event Selection Cuts . . . . . . . . . . . . 107

5.2 .2 Effect of Ratio Variation with Sectors . . . . . . . . . . . . . 108

5.2 .3 Effect of $e^{+} e^{-}$Luminosity Differences . . . . . . . . . . . 110

5.2 .4 Effect of Background Subtraction . . . . . . . . . . . . . . 113

5.2 .5 Total Systematic Uncertainties . . . . . . . . . . . . . . . 114

5.3 Comparison to the World Data and Theoretical Calculations . . . . . 114

5.4 Conclusions and Future Outlook . . . . . . . . . . . . . . . 116

\begin{tabular}{ll}
\hline Bibliography & 119
\end{tabular}

\begin{tabular}{ll}
\hline VITA & 124
\end{tabular} 


\section{LIST OF TABLES}

TABLE

PAGE

1.1 Properties of quarks . . . . . . . . . . . . . . . . 2

3.1 TPE standard experimental conditions . . . . . . . . . . . . . . . 62

3.2 Summary of the collected data . . . . . . . . . . . . . . 62

4.1 Summary of the Bad TOF paddles . . . . . . . . . . . . . . . . . . . 92

$4.2 \quad$ Average $\left\langle Q^{2}\right\rangle$ and $\langle\varepsilon\rangle$ for the binned data . . . . . . . . . . . . 96

5.1 Measured cross section ratio at high $\varepsilon$ bins before applying radiative corrections . . . . . . . . . . . . . . . . . . 104

5.2 Estimated systematic uncertainties due to various sources . . . . . . . 114

$5.3 \quad$ Final cross section ratio and the associated statistical $\left(\delta R_{\text {stat }}\right)$ and systematic uncertainties $\left(\delta R_{\text {sys }}\right) \ldots \ldots \ldots \ldots \ldots \ldots$ 


\section{LIST OF FIGURES}

FIGURE

PAGE

1.1 Valence quark composition of proton and neutron . . . . . . . . . . 3

1.2 Comparision between Rosenbluth and polarization transfer results . . 4

$2.1 \quad$ Feynman diagram showing elastic $e p$ scattering process $\ldots$. . . . . . . 7

2.2 Extraction of electric and magnetic form factor by the Rosenbluth separation method . . . . . . . . . . . . . . . 15

2.3 Electric $\left(G_{E}\right)$ and magnetic form factors $\left(G_{M}\right)$ scaled by dipole form factor $\left(G_{D}\right)[\ldots \ldots \ldots \ldots \ldots \ldots \ldots$

2.4 Comparison between Rosenbluth and polarization transfer results . . 19

2.5 The $\varepsilon$ dependence of the reduced cross section . . . . . . . . . . . 20

2.6 Feynman diagrams showing QED radiative corrections for elastic $e p$

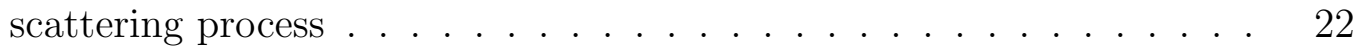

2.7 The ratio $Y_{2 \gamma}$ as a function of $\varepsilon$ for different values of $Q^{2}$. . . . . . . 25

2.8 The ratio of generalized form factors as obtained in Guichon and Vanderhaeghen analysis . . . . . . . . . . . . . . 26

2.9 The difference between the model independent and Mo and Tsai IR divergent contributions .................... 29

2.10 The BMT corrected form factor ratio as the function of $Q^{2}$. . . . . . 30

2.11 The handbag diagram for elastic lepton-quark scattering . . . . . . . 31

2.12 Form factor ratio before and after applying partonic TPE corrections to Rosenbluth data . . . . . . . . . . . . . . . . . . . . . 32

2.13 Previous world data for the ratio of $e^{+} p$ to $e^{-} p$ elastic scattering cross

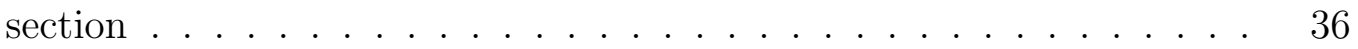

3.1 Schematic design of Jefferson lab accelerator . . . . . . . . . . . . . . 38

3.2 An exploded view of CLAS detector . . . . . . . . . . . . . . . . . 40

3.3 Magnetic field produced by the CLAS torus . . . . . . . . . . . 41

3.4 A vertical cut of the CLAS drift chambers through the target position. All the superlayers are shown. . . . . . . . . . . . . . . . . . . . 43

3.5 CLAS DC hexagonal drift cells . . . . . . . . . . . . . . . . . 44

3.6 View of TOF counters in one of CLAS sectors . . . . . . . . . . . . . 46

3.7 Exploded view of CLAS EC module. . . . . . . . . . . . . . . . . . . . . . . . . 47

3.8 Schematic diagram of the TPE beamline . . . . . . . . . . . . . . . . 48

3.9 Schematic diagram of the three dipole chicane . . . . . . . . . . . . . 49

3.10 Sparse Fiber Monitor . . . . . . . . . . . . . . . . . . . . . . . 51

3.11 Beam position distributions at SFM . . . . . . . . . . . . . . . . 52

3.12 The position of beam centroid position as a function of chicane current 53

3.13 Photograph of the actual TPE target cell . . . . . . . . . . . 54

3.14 Engineering assembly drawing of the TPE Target . . . . . . . . . . . 54 
3.15 TPE calorimeter module . . . . . . . . . . . . . . . . 56

3.16 Partially assembled TPE calorimeter . . . . . . . . . . . . . 57

3.17 Final design of the nominal shielding components for TPE Experiment 58

3.18 Photographs taken after the completion of beamline shielding work . $\quad 60$

3.19 Distribution of the residual in S1SL1. The black curve is the doubleGaussian fit to the residual distribution. . . . . . . . . . . . . . . . . 66

3.20 DC mean vs. run number . . . . . . . . . . . . . . . . . . 67

3.21 DC sigma vs. run number . . . . . . . . . . . . . . . . . 67

3.22 Energy loss correction for the proton tracks . . . . . . . . . . . . 68

3.23 Invariant mass distribution, $W$, before the momentum correction . . . 70

$3.24 \phi$ versus $\frac{\Delta p_{p}}{p}$ distribution before applying momentum correction . . . 71

$3.25 \phi$ versus $\frac{\Delta p_{p}}{p}$ distributions after applying momentum correction. . . . 74

3.26 Comparison of the invariant mass distributions before and after apply-

ing momentum corrections . . . . . . . . . . . . . . 75

4.1 Reconstructed target vertex distributions . . . . . . . . . . . . . . 78

$4.2 \quad$ Azimuthal angle $(\phi)$ vs. z-vertex $\left(v_{z}\right)$ distribution . . . . . . . . . . 78

4.3 Analysis flow chart . . . . . . . . . . . . . . . . . . 79

$4.4 \quad$ Azimuthal angle difference between lepton and proton . . . . . . . . . . 81

$4.5 \Delta E_{\text {beam }}$ vs. $\Delta E^{\prime}$ distributions for +- pairs (left) and ++ pairs (right). 83

$4.6 \Delta E_{\text {beam }}+\Delta E^{\prime}$ distributions $\ldots \ldots \ldots \ldots$. . . . . . . . . . 84

$4.7 \quad \Delta E_{\text {beam }}-\Delta E^{\prime}$ distributions $\ldots \ldots \ldots \ldots$. . . . . . . . . 85

$4.8 \Delta p_{\mathrm{p}}$ distributions $\ldots \ldots \ldots \ldots$. . . . . . . . . . . . . . 86

$4.9 \quad$ Azimuthal angle $(\phi)$ distributions at different scattering angles . . . . 87

4.10 The distribution of reconstructed in-bending electron tracks as a function of $\theta$ and $\phi \ldots \ldots \ldots \ldots \ldots$. . . . . . . . . . . . . . . . . 88

4.11 The distribution of reconstructed out-bending electron tracks as a function of $\theta$ and $\phi \ldots \ldots \ldots$. . . . . . . . . . . . . . . . . . . . 88

$4.12 \theta$-shift versus momentum of reconstructed electrons for positive torus

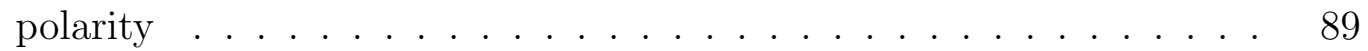

4.13 Reconstructed incident beam energy distributions for each chicane po-

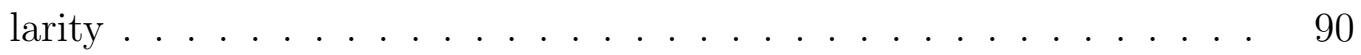

4.14 Reconstructed incident beam energy distributions for combined chicane

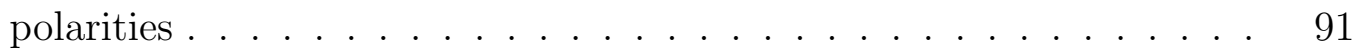

4.15 Swimming of the detected event through CLAS . . . . . . . . . . . 93

$4.16 Q^{2}$ vs. $\varepsilon$ distributions $\ldots \ldots \ldots \ldots$. . . . . . . . . . . . . . . . . . . . . . . . . 94

4.17 Binning schemes for the analysis . . . . . . . . . . . . . . . . . . 95

$4.18 \Delta \phi$ distribution at high $\varepsilon$ and low $Q^{2}$ bin for the events that pass all other kinematic cuts .................... 97

$4.19 \Delta \phi$ distribution at the lowest $\varepsilon$ and low $Q^{2}$ bin for the events that pass all other kinematic cuts. . . . . . . . . . . . . . . . 98

4.20 Subtraction of background by fitting the tails of $\Delta \phi$ distribution . . . 99

4.21 Single ratios for each torus polarity . . . . . . . . . . . . . . . . 100 
4.22 Double ratios for each chicane polarity . . . . . . . . . . . . . . . . 101

4.23 The ratio of positron energy density to the electron energy density as a function of the incident energy . . . . . . . . . . . . . . . . . . 102

5.1 Ratio of $e^{+} p$ to $e^{-} p$ yields at high $\varepsilon(\varepsilon>0.8)$, before radiative corrections. 104

5.2 Ratio of $e^{+} p$ to $e^{-} p$ yields at low $\left\langle Q^{2}\right\rangle \approx 0.85 \mathrm{GeV}^{2}$, before radiative

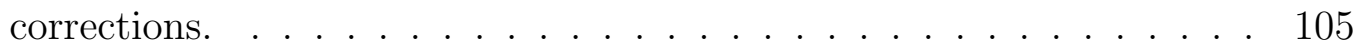

5.3 Measured cross section ratio as a function of $Q^{2}$ with and without radiative corrections at $\langle\varepsilon\rangle \approx 0.88 \mathrm{GeV}^{2} \ldots \ldots \ldots \ldots 6$

5.4 Measured cross section ratio as a function of $\varepsilon$ with and without radiative corrections at $\left\langle Q^{2}\right\rangle \approx 0.85 \mathrm{GeV}^{2}$. . . . . . . . . . . . . 106

5.5 Quadruple ratio as a function of $Q^{2}$ as measured by CLAS sectors . . 109

$5.6 \quad$ Quadruple ratio as a function of $Q^{2}$ for different cycles $\quad$. . . . . . . . 111

5.7 Torus polarity independent double ratio as a function of $Q^{2}$ for different magnet cycles . . . . . . . . . . . . . . . . . . 112

5.8 Cross section ratio at high $\varepsilon$ overlaid on the world data . . . . . . . . 116

5.9 Cross section ratio at low $Q^{2}$ overlaid on the world data. . . . . . . . 117

5.10 Cross section ratio at $Q^{2} \approx 1.45 \mathrm{GeV}^{2}$ overlaid on the world data . . 117 


\section{LIST OF ABBREVIATIONS}

ADC Analog to Digital Converter.

CEBAF Continuous Electron Beam Accelerator Facility.

CLAS CEBAF Large Acceptance Spectrometer.

QCD Quantum Chromodynamics.

QED Quantum Electrodynamics.

SRF Superconducting Radio Frequency.

TDC Time to Digital Converter.

TPE Two Photon Exchange. 


\section{Chapter 1}

\section{Introduction}

\subsection{Overview}

The fundamental goal of nuclear and particle physics is to understand the structure and behavior of the strongly interacting particles in terms of their building blocks. An important step towards the goal is the description of the internal structure of the proton and the neutron, collectively known as nucleons. Understanding the structure and dynamics of the nucleons has been the primary focus of many scientific efforts for almost a century now. The first hint about the composite nature of the nucleons was given by the discovery of the anomalous magnetic moment of the proton by $\mathrm{O}$. Stern in 1933 [1]. The measurement showed that the magnetic moment of the proton was surprisingly different from the expected value for a point-like Dirac particle with spin 1/2. Robert Hofstadter et al. in 1953 confirmed the composite nature of the nucleon by measuring the form factors in an electron scattering experiment [2], for which he was awarded the Nobel Prize in Physics in 1961. The pioneering work laid the foundation for the modern day picture of the nucleon.

In the standard model of the elementary particle physics, the nucleons are composed of quarks and the gluons. The quarks in the nucleons are bound together by the strong interaction, which is mediated by the exchange of vector gauge bosons known as gluons. Unlike the leptons, which carry only the electric charges, the quarks carry color charges and generate color forces. The strong interaction between the quarks and gluons is governed by theory of Quantum Chromodynamics (QCD). The quarks are elementary fermions and come in six different flavors, namely ' $u$ ' (up), 'd' (down), 'c' (charm), 's' (strange), 't' (top) and 'b' (bottom). The quarks can be grouped into 
three families in order of increasing mass as summarized in Table 1.1.

Table 1.1: Properties of quarks

\begin{tabular}{|c|c|c|c|}
\hline Generation & Flavor & Symbol & Charge \\
\hline First & up & $u$ & $+\frac{2}{3} e$ \\
& down & $d$ & $-\frac{1}{3} e$ \\
\hline Second & charm & $c$ & $+\frac{2}{3} e$ \\
& strange & $s$ & $-\frac{1}{3} e$ \\
\hline Third & top & $t$ & $+\frac{2}{3} e$ \\
& bottom & $b$ & $-\frac{1}{3} e$ \\
\hline
\end{tabular}

In $\mathrm{QCD}$, the quarks are confined, meaning the strong force between the quarks increases with distance. Because of the confinement property, a free quark has never been experimentally observed. The quarks always occur in bound states of colorless combinations into two families of particles, namely, the mesons and the baryons. The mesons are the bound states of a quark and anti-quark pair, while the baryons are the bound state of three valence quarks. The mesons and baryons form a larger family of particles called hadrons. The proton is a bound state of two up quarks and a down quark and the neutron is a bound state of one up quark and two down quarks. The valence quark composition of the proton and neutron are shown in Fig. 1.1. The proton and the neutron form an isospin doublet, meaning that the proton and neutrons are different quantum states of a single entity, the nucleon. The quarks move around inside the nucleon, so the nucleon has a charge distributed over its volume. This leads to the generation of an electric current, which in turn induces a magnetic field inside the nucleon. In addition, quarks and gluons both have spin, which contribute to the nucleon magnetic moment. The combination of the total magnetic field and the magnetic moment is called magnetization.

The electromagnetic interaction provides a basic tool to study the internal structure of the nucleons [3, 4, 5]. Experimentally, elastic electron-proton scattering, $p\left(e, e^{\prime}\right) p$ is one way to extract information about the internal structure of the proton. Elastic ep 

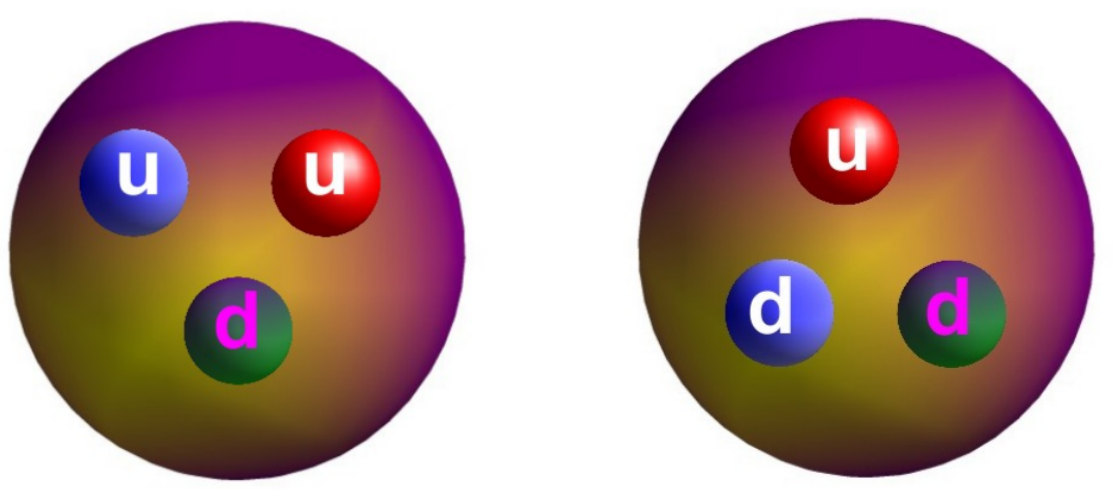

Figure 1.1: Quark composition of proton (left) and neutron (right)

scattering is the process in which the kinetic energy and momentum of the electron and proton is conserved and the proton remains intact after the collision. The cross section for the process can be described in terms of the electric $\left(G_{E}\right)$ and magnetic $\left(G_{M}\right)$ form factors. The form factors parameterize the deviation of the proton from a point-like particle and provide information about the composite nature of the proton. At low momentum transfer, they are related to the charge and magnetization distributions inside the proton. However, at high momentum transfer, they provide important information about the quark distributions within the nucleons as well as the nature of the strong force at moderate inter-quark separation [6]. The precise measurement of the electromagnetic form factors is key to our understanding of the internal structure of the proton. More discussion on this will be given in Chapter 2 . Over the past several decades, a large number of experiments have measured the electron-proton elastic scattering cross sections to extract the electric $\left(G_{E}\right)$ and the magnetic $\left(G_{M}\right)$ form factors of the proton using the Rosenbluth separation technique [8]. The ratio of the electric to magnetic form factors $\left(R=\frac{\mu_{p} G_{E}}{G_{M}}\right)$ measured by the Rosenbluth technique are consistent with an approximate form-factor scaling of $R \approx 1$ [6, 8, 9, 10, 11]. Here, $\mu_{p}$ is the magnetic dipole moment of the proton. 


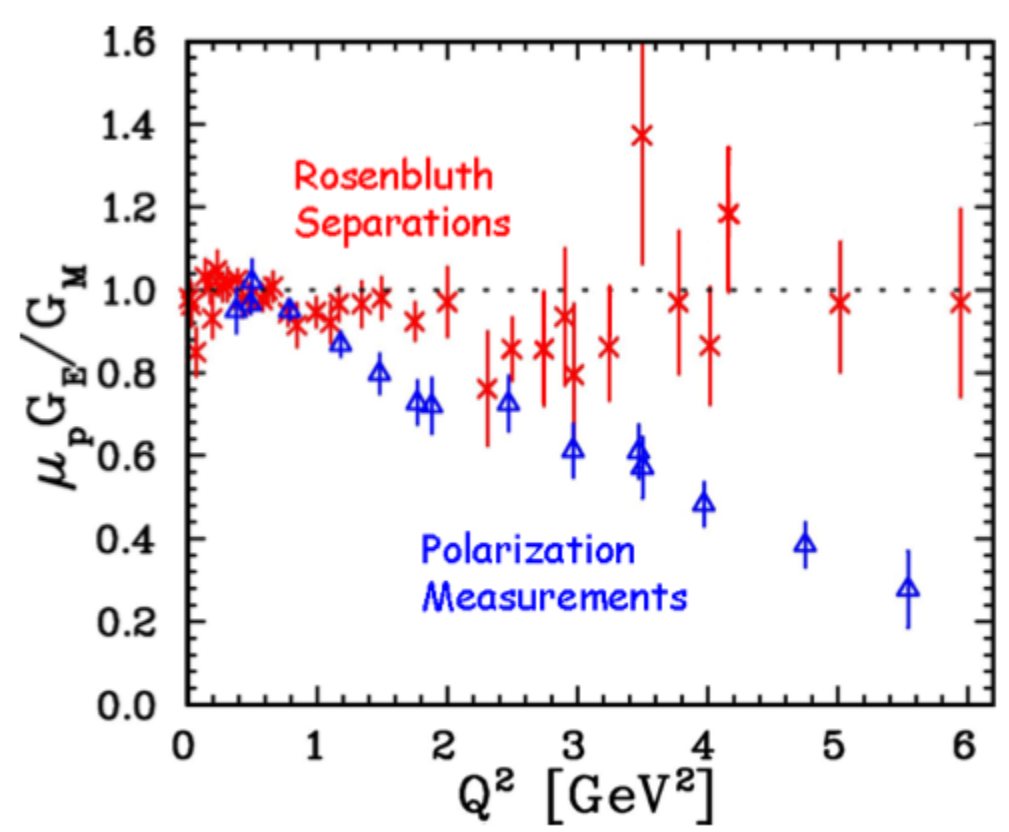

Figure 1.2: Comparison between Rosenbluth and polarization transfer results. The Rosenbluth results are shown in red and polarization transfer results are shown in blue. Figure adopted from Ref. [7]

The results indicate that the charge and the magnetization distribution inside the proton are similar. In contrast, the results from recent polarization transfer measurements [12, 13, 14, 15], using a polarized electron beam to measure the polarization of the recoil proton shows an approximately linear drop of the ratio from unity with increasing $Q^{2}$. The results from both techniques are shown in Fig. 1.2. Here, $Q^{2}$ is the four-momentum transferred by the incident electron to the proton. More details on the experimental measurement of the form factors can be found in Chapter 2 .

Clearly, the two results have a significant discrepancy that increases with $Q^{2}$. In the Born approximation, both methods assume the exchange of a single virtual photon between the electron and the nucleon during the scattering process. Many theoretical calculations suggest that higher-order contributions beyond the Born approximation, for example the contributions from the exchange of two virtual photons, may explain the discrepancy between the two methods [7, 16]. 
Calculations have shown that the two-photon exchange effects TPE can directly be extracted in a model-independent way by measuring the ratio of positron-proton $\left(e^{+} p\right)$ to electron-proton $\left(e^{-} p\right)$ elastic scattering cross sections [7, 16]. One such measurement was performed at experimental Hall B of Jefferson Lab using a combined electron-positron beam. The mixed electron-positron beam was created from the primary electron beam provided by CEBAF. The mixed beam was scattered off a liquid hydrogen target and the scattered particles were detected in CLAS. The main focus of this dissertation is the detailed description of the CLAS TPE experiment and its results.

The rest of this dissertation is organized as follows. Chapter 2 will lay the foundation for this dissertation discussing the basics of the form factors and their measurements, the discrepancy between the different methods of form factor measurements, possible resolution of the discrepancy, existing experimental evidence, and the theoretical calculations. The Chapter 3 will discuss the details of the experimental apparatus used during the measurement, data collection, and detector calibrations. The details of the data analysis procedures will be discussed in Chapter 4. The final results for the $e^{+} p$ and $e^{-} p$ cross-section ratio, including the systematic uncertainties, will be presented in Chapter 5. 


\section{Chapter 2}

\section{An Overview of the Proton Form Factors}

The form factors measure the deviation of a nucleon from a point-like particle. Hence they provide information about the composite nature of the nucleon. The electromagnetic form factors are the most basic observables that contain information about the spatial distribution of charge and magnetism inside the nucleon. In this dissertation, we will focus on the elastic electromagnetic form factors of the proton. This chapter will discuss the details of the proton's electromagnetic form factors and their measurements.

\subsection{Elastic Electromagnetic Form Factors}

\subsubsection{Kinematic Variables}

Elastic electron-nucleon scattering is a powerful experimental tool in the study of the internal structure of the nucleons. The electron being a structureless point charge, its magnetic moment is determined solely by its spin. Hence, the electromagnetic interactions of electrons with the nucleons are easy to understand within the framework of Quantum Electrodynamics QED. Thus, electron scattering is an ideal probe in studying the internal structure of the nucleons and the electromagnetic form factors [3, 4, 5]. The elastic electron-proton scattering process can be illustrated by the Feynman diagram shown in Fig. 2.1. In the Born approximation, the interaction takes place via the exchange of a single virtual photon ${ }^{1}$. The elastic ep scattering process can be represented in following nuclear reaction:

\footnotetext{
${ }^{1}$ An exchange particle of the electromagnetic interaction that transfers momentum from the electron to the proton
} 


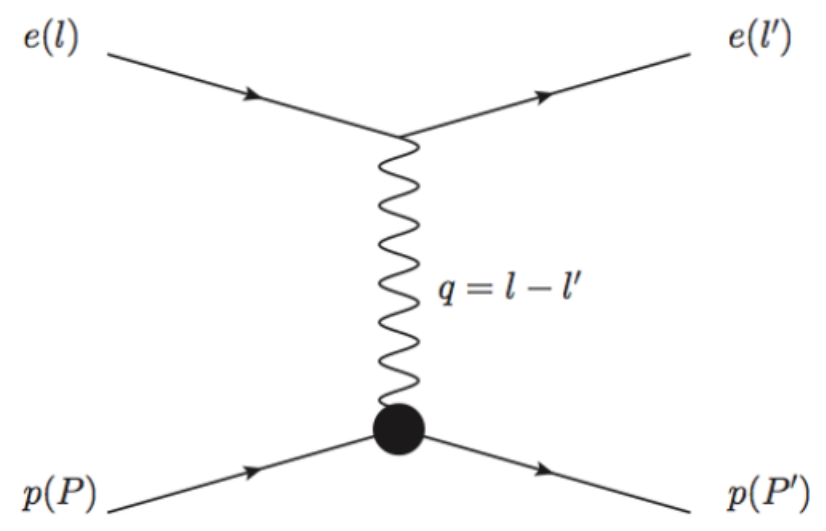

Figure 2.1: Feynman diagram showing elastic ep scattering process. The quantities in the brackets represent the four-momentum of the particle. $q$ is the four-momentum of the exchanged virtual photon.

$$
e\left(l_{\mu}\right)+p\left(P_{\mu}\right) \rightarrow e\left(l_{\mu}^{\prime}\right)+p\left(P_{\mu}^{\prime}\right) .
$$

Here, $e$ and $p$ refer to electron and the proton. In the lab frame:

- $l_{\mu}=(E, E \hat{\mathbf{z}})$ is the four-momentum of the incoming electron.

- $P_{\mu}=(M, 0)$ is the four-momentum of the target proton at rest.

- $l_{\mu}^{\prime}=\left(E^{\prime}, \mathbf{p}_{\mathbf{e}}^{\prime}\right)$ is the four-momentum of the scattered electron with momentum $\mathbf{p}_{\mathbf{e}}^{\prime}$ and energy $E^{\prime}$.

- $P_{\mu}^{\prime}=\left(E_{p}^{\prime}, \mathbf{p}_{\mathbf{p}}^{\prime}\right)$ is the four-momentum of the scattered proton with momentum $\mathbf{p}_{\mathbf{p}}^{\prime}$ and energy $E_{p}^{\prime}$. 
This dissertation will employ the so-called "natural units" used in particle physics. In the natural unit, the reduced Planck's constant $(\hbar)$ and the speed of light $(c)$ are taken as 1 i.e. $\hbar=c=1$. We will also assume that the mass of the electron is negligible $\left(m_{e} \approx 0\right)$ in comparison to the energies of the experiment. A four-vector will be denoted by italic type letter (e.g. $A^{\mu}$ ) and a three-vector will be denoted by boldface letter (e.g. A). For example, the four-momentum $\left(p^{\mu}\right)$ of a particle with energy $E$ and three-momentum $\mathbf{p}$ will be written as,

$$
p^{\mu}=(E, \mathbf{p}), \quad p_{\mu}=(E,-\mathbf{p})
$$

and their inner product,

$$
\begin{aligned}
p \cdot p & =p^{\mu} g_{\mu \nu} p^{\nu}=p^{\mu} p_{\nu} \\
& =E^{2}-|\mathbf{p}|^{2},
\end{aligned}
$$

gives the mass of the particle and is invariant under Lorentz transformation. The four-momentum of the exchanged virtual photon i.e., the four-momentum transfered by electron to the proton is given by:

$$
\begin{aligned}
q_{\mu} & =l_{\mu}-l_{\mu}^{\prime}=P_{\mu}^{\prime}-P_{\mu} \\
& =(\nu, \mathbf{q}),
\end{aligned}
$$

where $\nu$ and $\mathbf{q}$ are the energy and the three-momentum of the virtual photon. The four-momentum squared of the virtual photon is a Lorentz invariant quantity, which measures the resolution of the virtual photon and is given by:

$$
Q^{2}=-q^{2}=2 E E^{\prime}(1-\cos \theta),
$$


where $\theta$ is the scattering angle of the electron in the lab frame, $E$ is the energy of the incident electron and $E^{\prime}$ is the energy of the scattered electron. For the elastic scattering $E^{\prime}$ is given as,

$$
E^{\prime}=\frac{E M}{1+E(1-\cos \theta)},
$$

where $M$ is the proton mass. In Breit frame kinematics, where the energy transfer is always zero $(\nu=0)$, Eq. 2.7 yields,

$$
Q^{2}=-\mathbf{q}^{2} .
$$

We can also define another Lorentz invariant quantity,

$$
s=W^{2}=M^{2}+2 \nu M-Q^{2},
$$

which gives the invariant mass squared of the photon-proton final states. In elastic scattering, $W^{2}=M^{2}$.

Additionally, we can define two dimensionless variables $\tau$ and $\varepsilon$, which are equivalent to $Q^{2}$ and the scattering angle $\theta$,

$$
\tau=\frac{Q^{2}}{4 M^{2}}, \quad \varepsilon=\frac{\nu^{2}-\tau(1+\tau)}{\nu^{2}+\tau(1+\tau)} .
$$

The variable $\varepsilon$ is the measure of the longitudinal polarization of the virtual photon. In the target rest frame, $\varepsilon$ is given by,

$$
\varepsilon=\left(1+2(1+\tau) \tan ^{2}\left(\frac{\theta}{2}\right)\right)^{-1} .
$$




\subsubsection{Nucleon Form Factors: Basics}

Otto Stern et al. precisely measured the magnetic moment of the proton in the early 1930s [1]. The measured value was $\sim 2.8$ times higher than the expected value for a spin $\frac{1}{2}$ Dirac particle. The value suggested that proton cannot simply be a point charge and point magnetic moment but that it must have some internal structure. A measurement by Hofstadter et al. confirmed the composite nature of the proton by measuring its form factors [2]. The earliest model treated the proton as a neutron core embedded in a cloud of charged meson. The model described the form factors in terms of reduced charge and magnetic moments felt by the high-energy electrons while penetrating the mesonic cloud 8 .

Hofstadter et al. measured the phenomenological form factor squared as the deviation of the measured $e^{-} p$ elastic scattering cross section from the Mott cross section [2],

$$
\left|F\left(q^{2}\right)\right|^{2}=\frac{\left(\frac{d \sigma}{d \Omega}\right)_{e x p}}{\left(\frac{d \sigma}{d \Omega}\right)_{M o t t}} .
$$

The Mott cross section is the cross section for scattering of a spin $\frac{1}{2}$ electron from a point-like spinless particle, and is given as,

$$
\left(\frac{d \sigma}{d \Omega}\right)_{M o t t}=\left(\frac{\alpha}{2 E}\right)^{2}\left(\frac{\cos ^{2} \frac{\theta}{2}}{\sin ^{4} \frac{\theta}{2}}\right) \frac{E^{\prime}}{E},
$$

where $\alpha \approx \frac{1}{137}$ is the fine structure constant. In the non-relativistic limit, the form factor $F\left(q^{2}\right)$ can be written as the Fourier transform of the charge density distribution function as:

$$
F\left(Q^{2}\right)=\int_{V} \rho_{c h}(\mathbf{r}) e^{i \mathbf{q} \cdot \mathbf{r}} d^{3} \mathbf{r} .
$$


The elastic electromagnetic form factors contain all the details about the spatial distribution of the charge and magnetization inside the proton. The next section will set up a general formalism for the electromagnetic form factors associated with elastic electron-proton scattering.

\subsubsection{Elastic Electromagnetic Form Factors Formalism}

According to the Feynman rules for QED, the invariant amplitude for elastic electronproton scattering (See Fig. 2.1) in the leading order approximation is given as [17],

$$
\mathcal{M}_{1 \gamma}=-i \frac{e^{2}}{q^{2}} j_{\gamma \mu} J_{\gamma}^{\mu}
$$

where $e$ is the electronic charge, $j_{\gamma \mu}$ and $J_{\gamma}^{\mu}$ are the matrix elements of the lepton and fermion current operators. These can be written in terms of lepton $\left(u_{e}\right)$ and fermion $\left(u_{p}\right)$ spinors as,

$$
j_{\gamma \mu}=\bar{u}_{e}\left(l_{\mu}^{\prime}\right) \gamma_{\mu} u_{e}\left(l_{\mu}\right), \quad J_{\gamma}^{\mu}=\bar{u}_{p}\left(P_{\mu}^{\prime}\right) \Gamma^{\mu}(q) u_{p}\left(P_{\mu}\right) .
$$

The Lorentz invariant fermion current operator $\Gamma^{\mu}$ is defined in terms of Dirac $\left(F_{1}\right)$ and Pauli $\left(F_{2}\right)$ form factors as,

$$
\Gamma^{\mu}=F_{1}\left(Q^{2}\right) \gamma^{\mu}+i \frac{\kappa \sigma^{\mu \nu} q_{\nu}}{2 M} F_{2}\left(Q^{2}\right),
$$

where, $\sigma^{\mu \nu}=\frac{i}{2}\left[\gamma^{\mu}, \gamma^{\nu}\right]$ and $\kappa$ is the anomalous magnetic moment measured to be 1.79 nuclear magneton [18. The differential Born cross section is,

$$
\left(\frac{d \sigma}{d \Omega}\right)_{e x p}=\left(\frac{\alpha}{4 M Q^{2}} \frac{E^{\prime}}{E}\right)^{2}\left|M_{\gamma}\right|^{2}
$$


Upon solving Eq. 2.19.

$$
\left(\frac{d \sigma}{d \Omega}\right)_{e x p}=\left(\frac{d \sigma}{d \Omega}\right)_{M o t t}\left[\left(F_{1}^{2}+\kappa^{2} \frac{Q^{2}}{4 M^{2}} F_{2}^{2}\right)+\frac{Q^{2}}{2 M^{2}}\left(F_{1}+\kappa F_{2}\right)^{2} \tan ^{2} \frac{\theta}{2}\right] .
$$

The Sach's electric $\left(G_{E}\right)$ and magnetic $\left(G_{M}\right)$ form factors can be defined as the linear combinations of $F_{1}$ and $F_{2}$ as [19, 20],

$$
G_{E}\left(Q^{2}\right)=F_{1}\left(Q^{2}\right)-\kappa \tau F_{2}\left(Q^{2}\right), \quad G_{M}\left(Q^{2}\right)=F_{1}\left(Q^{2}\right)+\kappa F_{2}\left(Q^{2}\right) .
$$

The form factors are normalized in such a way that at $Q^{2}=0$, the electric and magnetic form factors give,

$$
G_{E}=1, \quad G_{M}=\mu_{p}
$$

where $\mu_{p}=2.793$ is the anomalous magnetic moment of the proton. Substituting Eq. 2.21 into Eq. 2.20 yields,

$$
\left(\frac{d \sigma}{d \Omega}\right)_{e x p}=\left(\frac{d \sigma}{d \Omega}\right)_{M o t t}\left[\frac{G_{E}^{2}\left(Q^{2}\right)+\tau G_{M}^{2}\left(Q^{2}\right)}{1+\tau}+2 \tau G_{M}^{2}\left(Q^{2}\right) \tan ^{2} \frac{\theta}{2}\right] .
$$

Eq. 2.23 is called the Rosenbluth or the Longitudinal-Transverse (LT) separation formula. In the non-relativistic limit $\left(Q^{2}<<M^{2}\right)$, the proton recoil and the energy transfer $(\nu)$ are negligible. Hence, we can take $q=(0, \mathbf{q})$ and $Q^{2}=|q|^{2}$. The Rosenbluth formula in Eq. 2.23 then reduces to,

$$
\left(\frac{d \sigma}{d \Omega}\right)_{\exp }=\left(\frac{d \sigma}{d \Omega}\right)_{M o t t} G_{E}^{2}\left(Q^{2}\right)
$$


Comparing Eq. 2.13, Eq. 2.15, and Eq. 2.24, $G_{E}\left(Q^{2}\right)$ and $G_{M}\left(Q^{2}\right)$ can be written as the Fourier transform of the proton's charge and magnetization distributions:

$$
\begin{aligned}
G_{E}\left(Q^{2}\right) & =\int_{V} \rho_{c h}(\mathbf{r}) e^{i \mathbf{q} \cdot \mathbf{r}} d^{3} \mathbf{r} \\
G_{M}\left(Q^{2}\right) & =\int_{V} \rho_{M}(\mathbf{r}) e^{i \mathbf{q} \cdot \mathbf{r}} d^{3} \mathbf{r} .
\end{aligned}
$$

Here, the integration is over all the volume. Expanding Eq. 2.26 in powers of q:

$$
\begin{aligned}
& G_{E}\left(Q^{2}\right)= \int_{0}^{\infty} \rho_{c h}(\mathbf{r})\left(1+i \mathbf{q} \cdot \mathbf{r}-\frac{(\mathbf{q} \cdot \mathbf{r})^{2}}{2}+\ldots\right) d^{3} \mathbf{r} \\
&= 2 \pi \int_{0}^{\infty} \rho_{c h}(\mathbf{r}) r^{2} d r \int_{0}^{\pi} \sin \theta d \theta\left(1+i|\mathbf{q}| r \cos \theta-\frac{q^{2} r^{2} \cos ^{2} \theta}{2}+\ldots\right)(2 . \\
& G_{E}\left(Q^{2}\right)=4 \pi \int_{0}^{\infty} \rho_{c h}(\mathbf{r}) r^{2} d r\left(1-\frac{q^{2} r^{2}}{6}\right)+\ldots \\
& G_{E}\left(Q^{2}\right)=1-\frac{1}{6} q^{2}\left\langle r^{2}\right\rangle+\ldots
\end{aligned}
$$

where, $\left\langle r^{2}\right\rangle=4 \pi \int_{0}^{\infty} r^{2} \rho(\mathbf{r}) r^{2} d r$ is the root mean square (RMS) charge radius of the proton. Differentiating $G_{E}$ with respect to $Q^{2}$ in the limit $Q^{2} \rightarrow 0$,

$$
\left.\frac{d G_{E}\left(Q^{2}\right)}{d Q^{2}}\right|_{Q^{2} \rightarrow 0}=-\frac{1}{6}\left\langle r^{2}\right\rangle
$$

Hence, the RMS charge radius of the proton is given as

$$
\left\langle r_{E}^{2}\right\rangle=-\left.6 \frac{d G_{E}\left(Q^{2}\right)}{d Q^{2}}\right|_{Q^{2} \rightarrow 0}
$$


A similar treatment of the magnetic form factor leads to the RMS magnetic radius given by,

$$
\left\langle r_{M}^{2}\right\rangle=-\left.6 \frac{d G_{M}\left(Q^{2}\right)}{d Q^{2}}\right|_{Q^{2} \rightarrow 0}
$$

The RMS charge and magnetic radius of the proton have been determined to be $\left\langle r_{E}\right\rangle=$ $0.879 \pm 0.0008 \mathrm{fm}$ and $\left\langle r_{m}\right\rangle=0.777 \pm 0.00017 \mathrm{fm}$ respectively, by analyzing data from electron scattering experiments [21]. The RMS charge radius of the proton determined from the Lamb shift in muonic hydrogen atom yields $0.84184 \pm 0.00067 \mathrm{fm}$ [22], which differs from the CODATA10 [23] value of $0.8775 \pm 0.00051 \mathrm{fm}$. The apparent difference has led to the so called "proton radius puzzle" and several activities are going on in order to understand and resolve the puzzle. It has been suggested that the two-photon exchange effect, which is the subject of the dissertation, can have a large impact on the extraction of charge and magnetic radius from the form factors [24].

\subsection{Measurements of Electromagnetic Form Factors in the Born Approximation}

Over the past several decades the standard method to extract the proton's electric and magnetic form factors has been the Rosenbluth, or L-T separation method [6, 8, 9, 10]. The method involves extracting the electric and magnetic form factors from the Born cross section by varying the incident electron energy and the detection angle for the scattered electron at fixed $Q^{2}$. More recent methods employ polarization degrees of freedom. In these methods, the electric to magnetic form factor ratio is determined by measuring the longitudinal and transverse polarization transferred to the proton by the polarized electron beam [12, 13, 14, 15]. Both of these methods assume an exchange of a single virtual photon in the scattering process. This section gives more insight into the details of these two methods. 


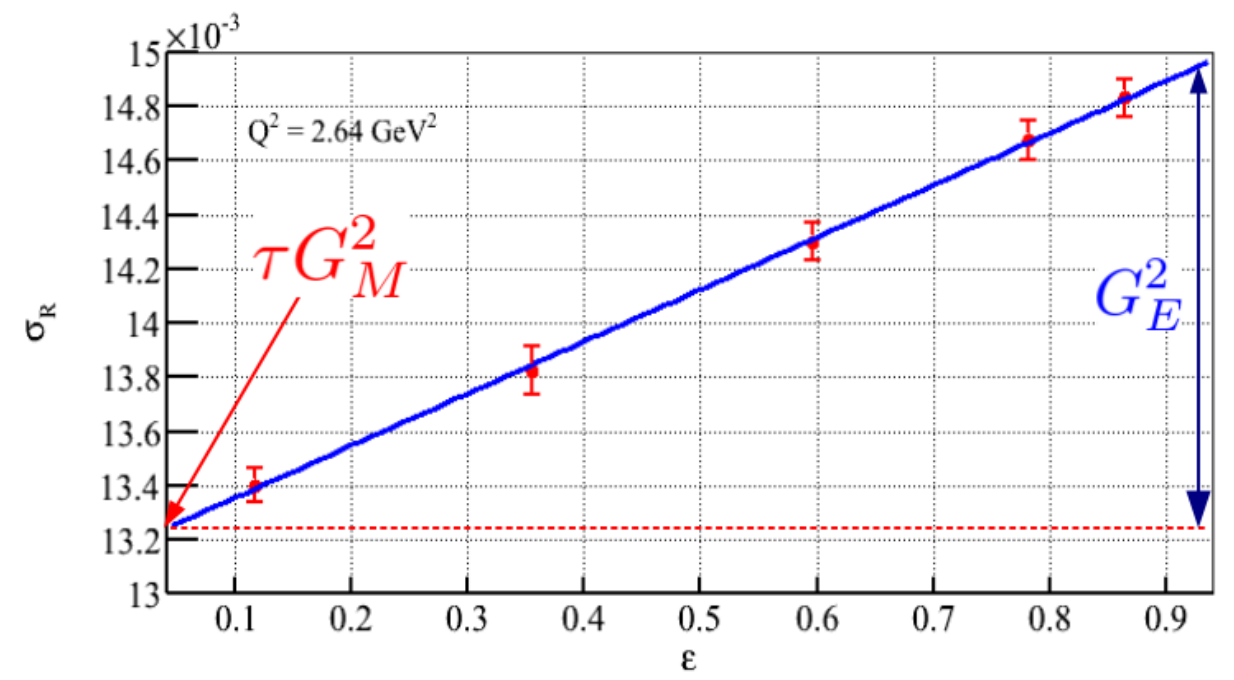

Figure 2.2: Extraction of electric and magnetic form factor by the Rosenbluth separation method. Solid line is a linear fit to the data points. The data are from Ref. [25]

\subsubsection{Rosenbluth Separation Method}

The Rosenbluth separation technique has been the standard method of extracting the form factors for several decades. Rearranging the terms in Eq. 2.23, the reduced cross section $\left(\sigma_{R}\right)$ can be written as,

$$
\sigma_{R}=\left[\varepsilon G_{E}^{2}\left(Q^{2}\right)+\tau G_{M}^{2}\left(Q^{2}\right)\right]
$$

where

$$
\sigma_{R}=\left(\frac{d \sigma}{d \Omega}\right)_{e x p} \varepsilon(1+\tau) /\left(\frac{d \sigma}{d \Omega}\right)_{M o t t}
$$

Since Born level cross sections are functions of $Q^{2}$ only, it is obvious from Eq. 2.34 that the reduced elastic scattering cross section is linearly dependent on $\varepsilon$ at fixed $Q^{2}$. 
Experimentally, the reduced cross section is measured at fixed $Q^{2}$ while varying $\varepsilon$ by varying the electron scattering angle and the energy of the incident electron. Fig. 2.2 shows a plot of $\sigma_{R}$ as the function of $\varepsilon$ at fixed $Q^{2}=2.64 \mathrm{GeV}^{2}$ from a Jefferson Lab measurement using the Rosenbluth separation method [11]. The straight line is the best fit to the experimental data. The electric $\left(G_{E}\right)$ and magnetic $\left(G_{M}\right)$ form factors are then obtained from the slope and the intercept of the $\varepsilon$-dependence of $\sigma_{R}$ after correcting for the radiative processes described in the next section.

The electric and the magnetic form factors have been extracted up to $Q^{2}$ of several $\mathrm{GeV}^{2}$ by the Rosenbluth separations. It can be observed from Eq. 2.34 that both $G_{M}$ and $G_{E}$ terms contribute to the cross section except when $\varepsilon=0$. $G_{M}$ is the only term that contributes to the cross section when $\varepsilon=0$. Since $\tau \propto Q^{2}$ and $\varepsilon \propto \frac{1}{Q^{2}}$, it is clear from Eq. 2.34 that the contribution of $G_{E}$ and $G_{M}$ to $\sigma_{R}$ is purely dependent on the value of $Q^{2}$. At low $Q^{2}$, the contribution from $G_{E}^{2}$ is dominant over the contribution from $G_{M}^{2}$. However, at high $Q^{2}$, the contribution from $G_{M}^{2}$ dominates over $G_{E}^{2}$ making the precise measurement of $G_{E}^{2}$ difficult.

The extracted $G_{E}$ and $G_{M}$ from different experiments using Rosenbluth separation methods are shown in Fig. 2.3 . Both $G_{E}$ and $G_{M}$ are scaled by the dipole form factor $G_{D}$ given by,

$$
G_{D}=\frac{1}{\left(1+Q^{2} / 0.71\right)^{2}}
$$

Fig. 2.3 illustrates that $G_{E}$ follows dipole scaling up to $Q^{2} \approx 1 \mathrm{GeV}^{2}$, which is not apparent at higher $Q^{2}$. However, $G_{M}$ shows internal consistency up to $Q^{2}$ of several $\mathrm{GeV}^{2}$. 

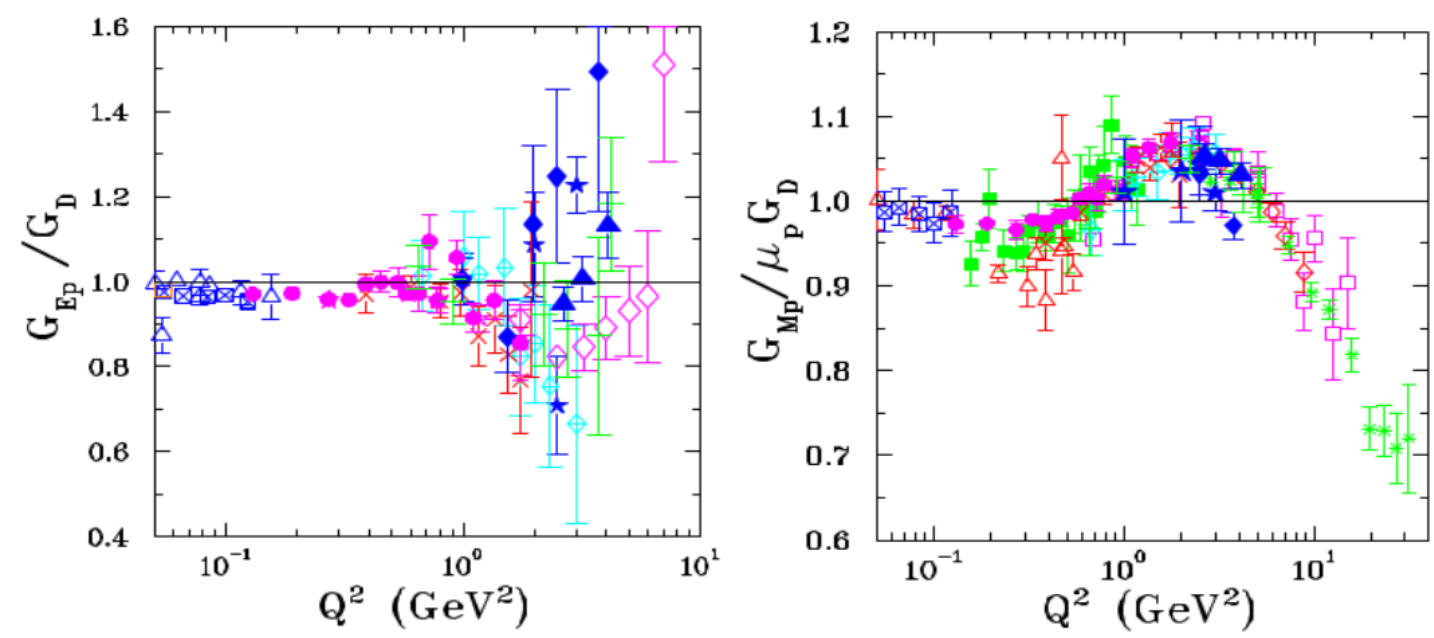

Figure 2.3: Extracted electric $\left(G_{E}\right)$ and magnetic $\left(G_{M}\right)$ form factors from from different experiments using Rosenbluth separation method. The form factor have been scaled by the dipole form factor $\left(G_{D}\right)$. Figure adopted from Ref. [4].

\subsubsection{Polarization Transfer (PT) Method}

More recent methods of the form factor measurements, known as the "polarization transfer methods", utilize polarization degrees of freedom in $p\left(\vec{e}, e^{\prime}\right) \vec{p}$ scattering to measure the electric to magnetic form factor ratio $\left(R=\frac{\mu_{p} G_{E}}{G_{M}}\right)$. In the method, longitudinally polarized electrons are scattered from an unpolarized proton target. In the one-photon exchange approximation, the longitudinal and transverse components of the polarization transfered to the struck proton are measured. The ratio of transverse to longitudinal components of the polarization vector are given by [26]:

$$
\frac{P_{t}}{P_{l}}=-\mu_{p} \frac{2 M}{E+E^{\prime}} \frac{G_{E}}{G_{M}} \frac{1}{\tan \left(\frac{\theta}{2}\right)}
$$

which yields,

$$
\frac{\mu_{p} G_{E}}{G_{M}}=-\frac{E+E^{\prime}}{2 M} \tan \left(\frac{\theta}{2}\right) \frac{P_{t}}{P_{l}}
$$


A number of recent experiments at Jefferson Lab and MIT-Bates [12, 13, 14, 15, 27, 28] have measured the proton form factor ratio $R$ up to $Q^{2}=8.5 \mathrm{GeV}^{2}$. The results from both the polarization transfer and the Rosenbluth separation methods are presented in Fig. 2.4. It is clear that the polarization results show a linear decrease of $R$ with $Q^{2}$ while the results from the Rosenbluth methods show $R \approx 1$ over the full range of $Q^{2}$, though with larger uncertainties at higher $Q^{2}$. The discrepancy between the two methods increases with $Q^{2}$. The contrasting results from the two methods led to the "Proton Form Factor Puzzle." The next sections will discuss the details of the puzzle along with attempts to reconcile the results from the two methods.

\subsection{Proton Form Factor Puzzle}

Even though both the Rosenbluth separation and the polarization transfer methods rely on the Born approximation, the ratio of form factors $\left(R=\frac{\mu_{p} G_{E}}{G_{M}}\right)$ measured by the two methods are in serious disagreement, especially at large $Q^{2}$. In the Born approximation, the cross section is calculated by considering only a single virtual photon exchanged between the lepton and the proton. There is a sharp disagreement between the results from the two methods as shown in Fig. 2.4, even after applying the standard radiative corrections [29]. This apparent discrepancy between the two measurements casts a serious doubt on our understanding of the nucleon structure. Our knowledge of the charge and magnetization distribution inside proton will not be complete until the cause of this discrepancy is fully understood. Hence, the discovery of the "form factor puzzle" in the late 1990's led to significant scientific activity intended to better understand and resolve the cause of this discrepancy.

Early on the cause of the discrepancy was thought to be related to the large systematic errors associated with the Rosenbluth measurements at higher $Q^{2}$. Improved Rosenbluth measurements were made at Jefferson Lab with a precision comparable to that of the polarization transfer methods [11, 25]. These results confirmed earlier 


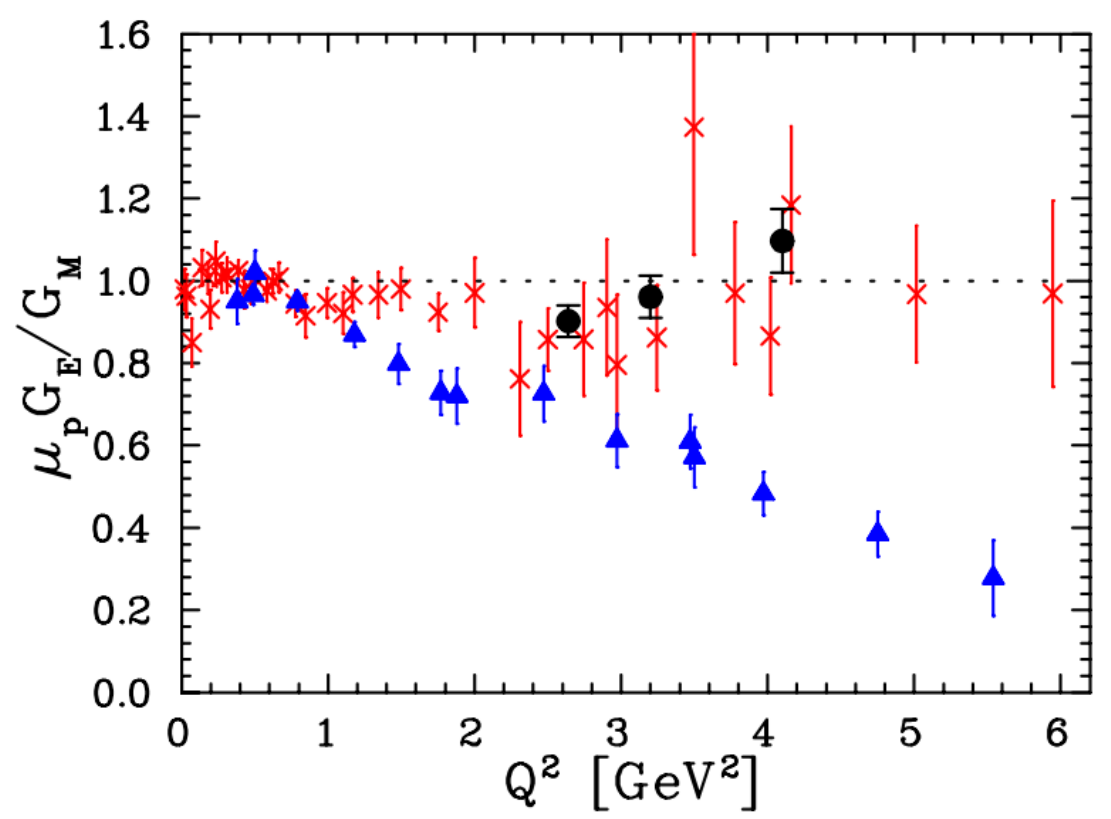

Figure 2.4: Comparison between Rosenbluth and polarization transfer results. The Rosenbluth results are shown in red and polarization transfer results are shown in blue. Figure adopted from Ref. [30]

Rosenbluth measurements indicating some fundamental cause for the discrepancy. In the mean time, a global reanalysis of the available cross section data was performed by Arrington [7]. The relative normalization of different datasets used in previous form factor extractions were modified and the radiative corrections for some measurements were updated. The extracted form factor ratios $\frac{\mu_{p} G_{E}}{G_{M}}$ were still in significant disagreement with the polarization transfer measurements. The analysis did not find any serious inconsistency in the previous Rosenbluth extractions. It confirmed that the discrepancy was indeed real and suggested that there should be some fundamental reason behind the discrepancy [7].

A very small error in the cross section measurement can have a significant impact on the form factor extraction by the Rosenbluth method. Roughly, an error of 5$10 \%$ at $Q^{2}>2-3 \mathrm{GeV}^{2}$ in the cross section measurement would be sufficient to explain the discrepancy [7]. Fig. 2.5 shows the slope of the reduced cross section as 


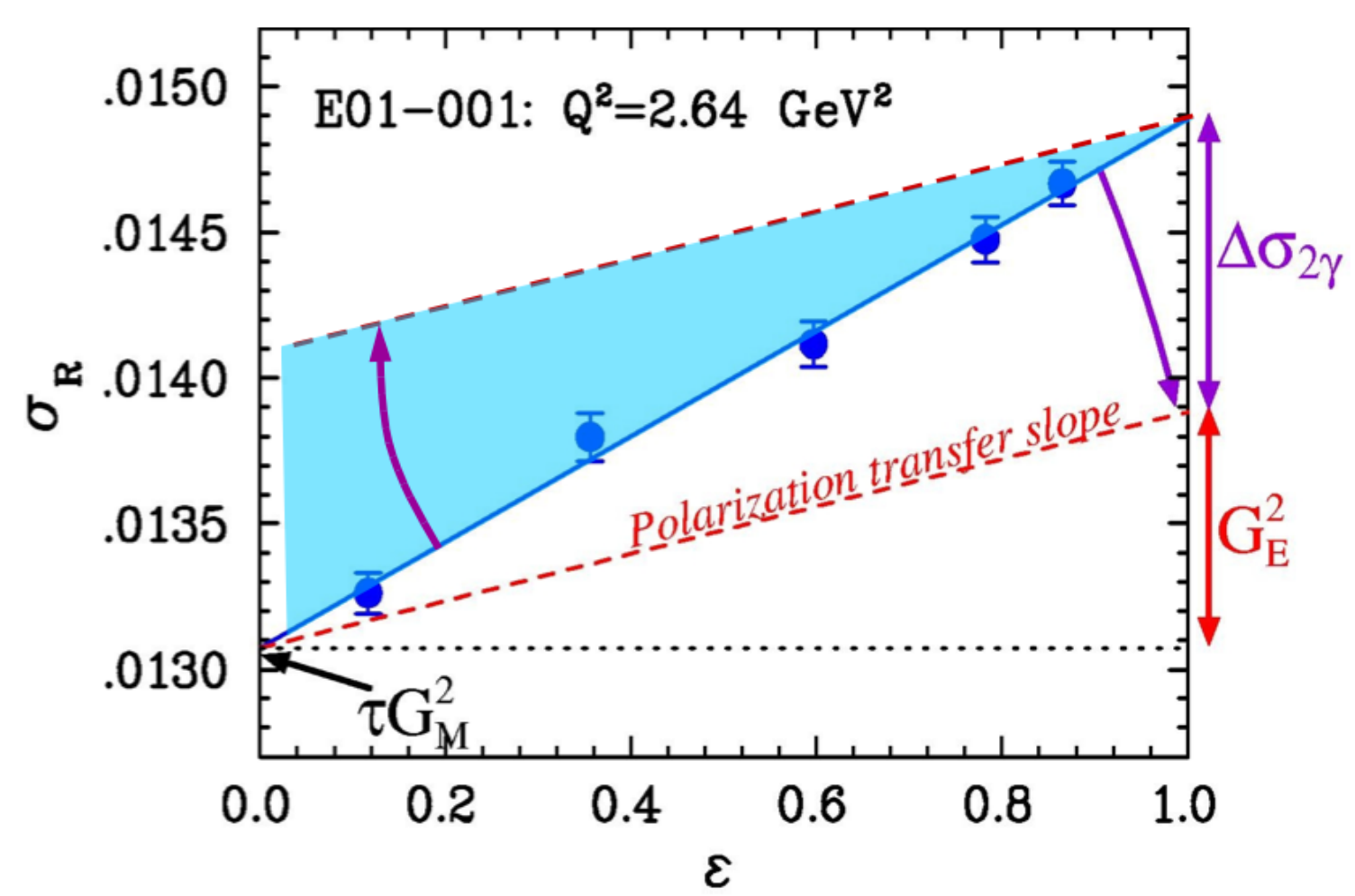

Figure 2.5: The $\varepsilon$ dependence of the reduced cross section as measured in high precision Rosenbluth measurement (Blue circles). Blue solid line is the best fit to the data. The red dashed line is the slope expected from the polarization transfer measurements. Figure adopted from Ref. [30]

measured by the Jefferson Lab high-precision Rosenbluth experiment [11] and also the slope expected from the polarization transfer measurements in the one-photon exchange approximation. If the polarization transfer measurements represent the correct form factors, then the dashed line would represent the actual slope with the correct contribution from the electric form factor [7]. The difference between the slope of the Rosenbluth data and the slope suggested by the polarization transfer data may possibly be a result of effects beyond Born approximation. 


\subsection{Possible Resolution of the Puzzle: Two Photon Exchange Effects}

As soon as the form factor discrepancy was verified, focus then turned to find the cause of this discrepancy and the methods to reconcile the results. After a global analysis of the previous cross section data did not find any inconsistencies in the datasets [7], the higher order effects beyond the Born approximation were considered as the explanation for the discrepancy. The two-photon exchange effects usually ignored in the standard treatment of radiative corrections [29, 31] were suggested as the likeliest explanation for the discrepancy [16, 32, 33].

The higher order QED radiative corrections shown in Fig. 2.6 modify the reduced Born level elastic scattering cross sections as:

$$
\sigma=\sigma_{R}(1+\delta),
$$

The correction term $\delta$ can be written as,

$$
\delta=\delta_{\text {virt }}+\delta_{\text {brem }}
$$

where $\delta_{\text {virt }}$ includes contribution from the exchange of another virtual photon (diagrams (a), (b), (e), (f), and (g)) while $\delta_{\text {brem }}$ includes contributions from the inelastic bremsstrahlung processes (diagrams (c), (d), and (h)). Usually the contributions from all the diagrams except for the TPE effects (diagrams (e) and (f)) are accounted

for in the standard radiative corrections. The TPE effects are generally neglected in these corrections as the calculation of these effects require the knowledge of overall response of the nucleon to the virtual photon. The contribution from the inelastic bremsstrahlung corrections $\delta_{\text {brem }}$ are also included as a correction to the experimental 


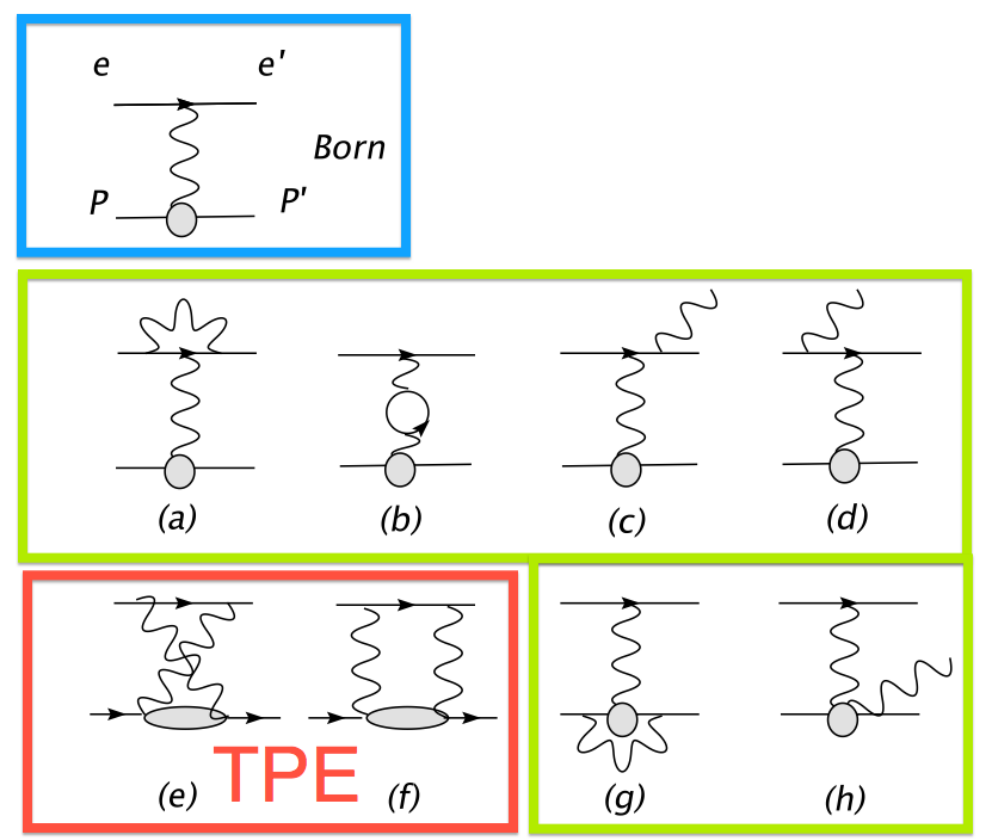

Figure 2.6: Feynman diagrams showing QED radiative corrections for elastic $e^{-} p$ scattering process. Here, the top-most diagram shows the Born term, diagrams (a) and $(\mathrm{g})$ show the vertex renormalization terms for the electron and the proton respectively, diagram (b) shows the loop diagram for vacuum polarization, diagrams (c) and (d) show the electron bremsstrahlung terms, and diagram (h) shows proton bremsstrahlung term. The diagrams (e) and (f) show the two-photon exchange terms that are usually neglected in the standard radiative corrections for elastic $e^{-} p$ scattering.

cross section.

The form-factor discrepancy has been interpreted as the failure of the one photon exchange approximation [32]. Hence, the higher order corrections to the Born level cross section such as the exchange of two or more hard photons between the lepton and the proton was considered. Previous calculations of such higher order corrections were found to be very small, typically below $1 \%$ of the Born cross section [34, 35]. These calculations were based on nucleon and resonance intermediate states and are valid only at lower $Q^{2}$ and are unknown at higher $Q^{2}$ [32]. Precise understanding of the $\varepsilon$ dependence of these radiative corrections is required at higher $Q^{2}$. A number of theoretical calculations were performed in order to estimate the size of the corrections 
necessary to resolve the discrepancy [16, 32, 33]. The next section will discuss different theoretical calculations of the TPE corrections.

\subsection{Theoretical Calculations of TPE}

Beyond the one photon exchange approximation, the response of the proton to the exchanged virtual photon is not well understood. Hence, the calculations of the two-photon exchange box and crossed diagrams shown in Fig. 2.6 (diagrams (e) and (f)), become extremely complicated as these calculations require knowledge of all the excited states of the proton. As a result, several theoretical models have been incorporated to compute the two-photon exchange diagrams. These calculations are mostly model-dependent and yield significantly different results for the extracted form factors.

\subsubsection{Guichon and Vanderhaeghen Calculation}

Guichon and Vanderhaeghen investigated the TPE effect by using the full $e^{-} p$ elastic scattering amplitude rather than approximating it to the Born level [32]. Beyond the Born approximation, the full amplitude $\mathcal{M}$ for elastic scattering of an electron from

a proton can be described in terms of three generalized form factors, $\tilde{F}_{1}, \tilde{F}_{2}$ and $\tilde{F}_{3}$, as 32 ,

$$
\mathcal{M}=-i \frac{e^{2}}{Q^{2}} \bar{u}\left(k^{\prime}\right) \gamma_{\mu} u(k) \bar{u}\left(p^{\prime}\right)\left[\tilde{F}_{1} \gamma^{\mu}+\tilde{F}_{2} \frac{i \sigma^{\mu \nu} q_{\nu}}{2 M}+\tilde{F}_{3} \frac{\gamma \cdot K P^{\mu}}{M^{2}}\right] u(p)
$$

where $K=\frac{k+k^{\prime}}{2}$ and $P=\frac{p+p^{\prime}}{2} . \tilde{F}_{1}, \tilde{F}_{2}$, and $\tilde{F}_{3}$ are generalized form factors, and are complex functions of $\nu=K \cdot P$ and $Q^{2}$. In the Born approximation, the generalized form factors $\tilde{F}_{1}$ and $\tilde{F}_{2}$ must reduce to Dirac and Pauli form factors, and the 
generalized form factor $\tilde{F}_{3}$ must vanish, i.e

$$
\begin{aligned}
& \tilde{F}_{1}^{\text {Born }}\left(\nu, Q^{2}\right) \rightarrow F_{1}\left(Q^{2}\right) \\
& \tilde{F}_{2}^{\text {Born }}\left(\nu, Q^{2}\right) \rightarrow F_{2}\left(Q^{2}\right) \\
& \tilde{F}_{3}^{\text {Born }}\left(\nu, Q^{2}\right) \rightarrow 0 .
\end{aligned}
$$

The Sach's electric $\left(\tilde{G_{E}}\right)$ and magnetic $\left(\tilde{G_{M}}\right)$ form factors are modified from the Born level form factors, i.e

$$
\begin{aligned}
\tilde{G_{E}} & =G_{E}+\Delta G_{E} \\
\tilde{G_{M}} & =G_{M}+\Delta G_{M}
\end{aligned}
$$

The generalized form factors are not observables and hence they do not have any intrinsic physical meaning. Their magnitude and the $\varepsilon$ dependence depend upon the choice of parametrization [36]. Considering only up to the order $\alpha^{2}$, the reduced cross section can be written as [32],

$$
\sigma_{R} \approx\left[\tau+\varepsilon \frac{\left|\tilde{G_{E}}\right|^{2}}{\left|\tilde{G_{M}}\right|^{2}}+2 \varepsilon\left(\tau+\frac{\left|\tilde{G_{E}}\right|}{\left|\tilde{G_{M}}\right|}\right) \operatorname{Re}\left(\frac{\nu \tilde{F_{3}}}{M^{2}\left|\tilde{G_{M}}\right|}\right)\right]
$$

and the polarization transfer ratio modifies to [32]:

$$
\frac{P_{t}}{P_{l}} \approx-\sqrt{\frac{2 \varepsilon}{\tau(1+\tau)}}\left[\frac{\left|\tilde{G_{E}}\right|}{\left|\tilde{G_{M}}\right|}+\left(1-\frac{2 \varepsilon}{1+\varepsilon} \frac{\left|\tilde{G_{E}}\right|}{\left|\tilde{G_{M}}\right|}\right) \operatorname{Re}\left(\frac{\nu \tilde{F_{3}}}{M^{2}\left|\tilde{G_{M}}\right|}\right)\right] .
$$

The dimensionless quantity,

$$
Y_{2 \gamma}\left(\nu, Q^{2}\right)=\operatorname{Re}\left(\frac{\nu \tilde{F}_{3}}{M^{2}\left|\tilde{G_{M}}\right|}\right),
$$




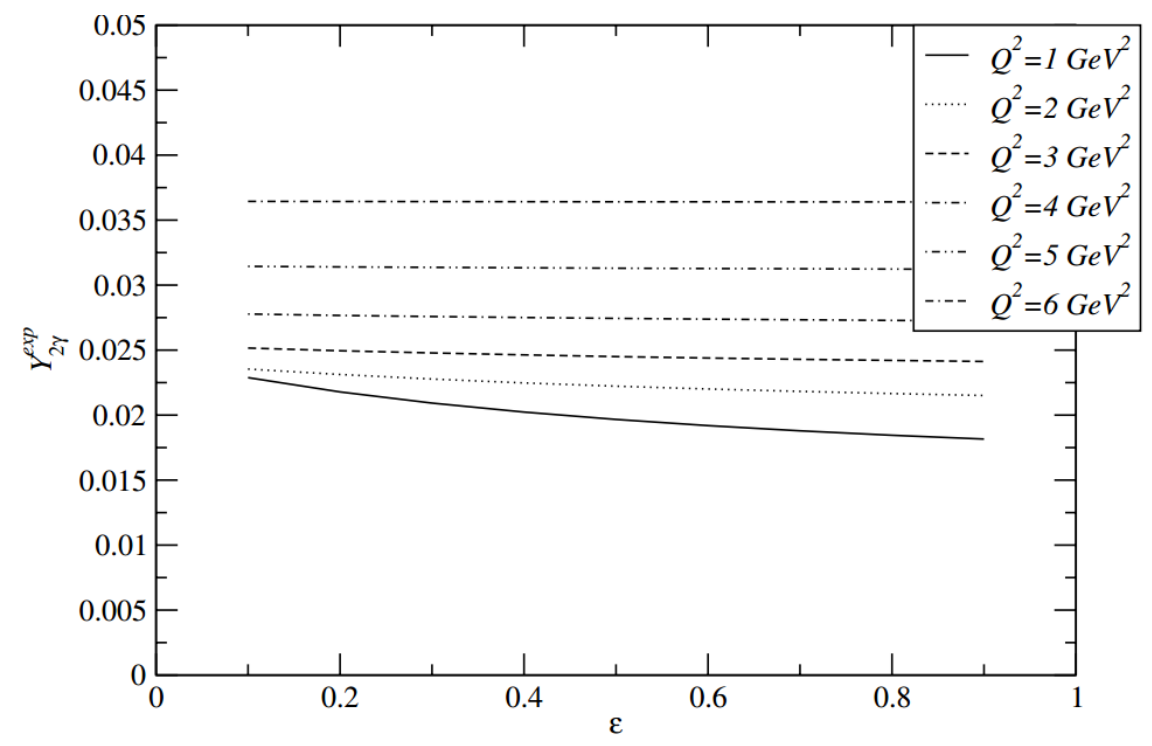

Figure 2.7: The ratio $Y_{2 \gamma}$ as a function of $\varepsilon$ for different values of $Q^{2}$. Figure adopted from Ref. [32]

measures the size of the two-photon exchange effect. Comparing the modified Rosenbluth Eq. 2.47 with the Born level Rosenbluth Eq. 2.34, we can immediately see that the experimentally measured ratio using the Rosenbluth method is actually,

$$
\left(R_{\text {Rosenbluth }}^{\text {exp }}\right)^{2}=\frac{\left|\tilde{G_{E}}\right|^{2}}{\left|\tilde{G_{M}}\right|^{2}}+2\left(\tau+\frac{\left|\tilde{G_{E}}\right|}{\left|\tilde{G_{M}}\right|}\right) Y_{2 \gamma} .
$$

Similarly comparing the modified polarization transfer ratio with the Born level polarization transfer ratio, it is clear that the experimentally measured ratio is,

$$
\left(R_{\text {polarization }}^{\text {exp }}\right)=\frac{\left|\tilde{G_{E}}\right|}{\left|\tilde{G_{M}}\right|}+\left(1-\frac{2 \varepsilon}{1+\varepsilon} \frac{\left|\tilde{G_{E}}\right|}{\left|\tilde{G_{M}}\right|}\right) Y_{2 \gamma}
$$

Eq. 2.50 and Eq. 2.51 were solved numerically by fitting the experimental data [9] with a polynomial function neglecting the effect of error bars. The solution of Eq. 2.50 and Eq. 2.51 for the ratio $Y_{2 \gamma}^{\text {exp }}$ as a function of $\varepsilon$ for different $Q^{2}$ are shown in Fig. 2.7. The ratios are on the order of a few percent in magnitude and are roughly independent of $\varepsilon$. With these corrections, the Rosenbluth and the polarization method both yield the 


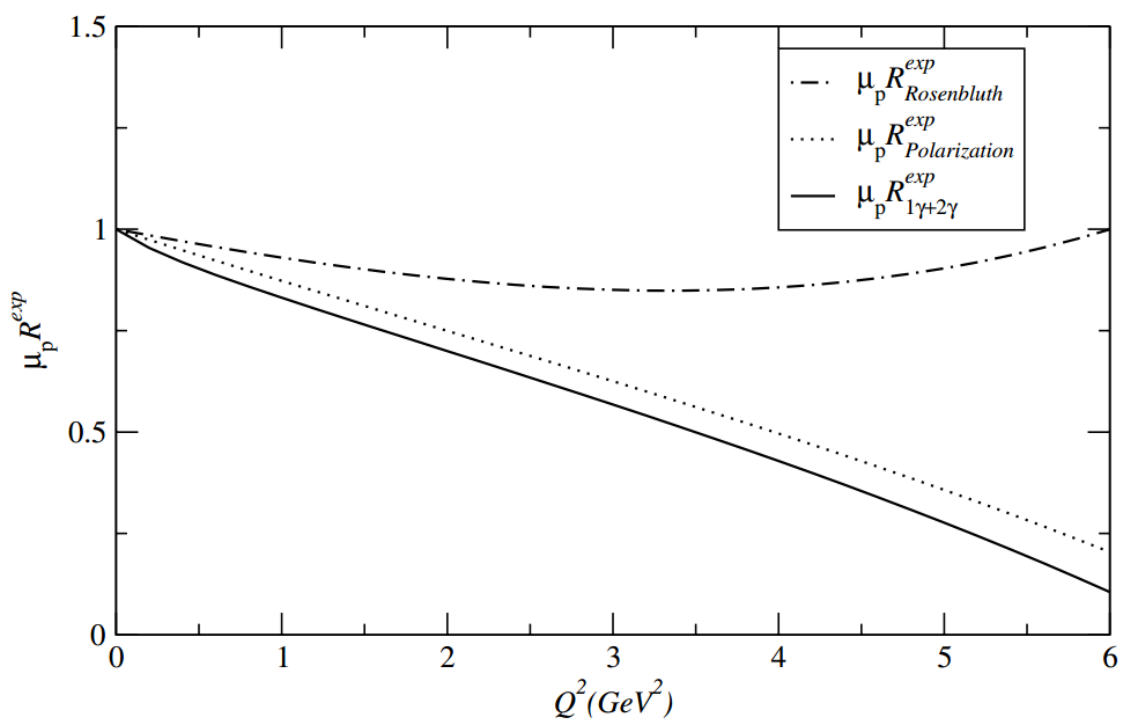

Figure 2.8: Form factor ratios deduced from Guichon and Vanderhaeghen analysis compared to experimental fits to the Rosenbluth and polarization transfer data. Figure adopted from Ref. 32

same value for the modified form factor ratio $\frac{\left|\tilde{G_{E}}\right|}{\left|\tilde{G_{M}}\right|}$. Fig. 2.8 shows the comparison of fits to the experimental form factor ratios measured by Rosenbluth and polarization transfer with that to the modified ratio. It illustrates that the form factor ratio modified by the two-photon exchange term is closer to the ratio measured by the polarization method in the one-photon exchange approximation.

\subsubsection{BMT Calculation}

In an attempt to reconcile the Rosenbluth and polarization transfer results, Blunden, Melnitchouk, and Tjon evaluated the TPE box and crossed diagrams shown in Fig. 2.6 (diagrams (e) and (f)) using a hadronic intermediate state model [37]. They wrote the amplitude for the one-loop virtual corrections $\mathcal{M}_{1}$ as the sum of a factorizable term that is proportional to the Born amplitude $\mathcal{M}_{0}$ and a non-factorizable term $\overline{\mathcal{M}}_{1}$ 
i.e.

$$
\mathcal{M}_{1}=f\left(Q^{2}, \varepsilon\right) \mathcal{M}_{0}+\overline{\mathcal{M}}_{1}
$$

The factorizable part, parameterized by $f\left(Q^{2}, \varepsilon\right)$ contains hadron-structure independent terms. However, the non-factorizable term $\overline{\mathcal{M}}_{1}$ depends upon the hadron structure. From Eq. 2.39, the ratio of the full to the Born level cross section is,

$$
1+\delta=\frac{\sigma}{\sigma_{0}}=\frac{\left|\mathcal{M}_{0}+\mathcal{M}_{1}\right|^{2}}{\left|\mathcal{M}_{0}\right|^{2}}
$$

To first order in $\alpha$, the total correction $\delta$ is given by,

$$
\delta \approx 2 f\left(Q^{2}, \varepsilon\right)+2 \frac{\operatorname{Re}\left\{\mathcal{M}_{0} \overline{\mathcal{M}}_{1}\right\}}{\left|\mathcal{M}_{0}\right|^{2}}
$$

Most of the corrections due to hadron-structure independent terms $\left(f\left(Q^{2}, \varepsilon\right)\right)$ are generally included in the standard radiative corrections of Mo and Tsai [29, 31] leaving out the hadron structure dependent terms. The BMT model considered the hadronstructure dependent corrections such as two-photon exchange and the finite proton vertex correction and are included in $\overline{\mathcal{M}}_{1}$. The finite proton vertex correction does not contribute to the form factor discrepancy because it was found to be less than $0.5 \%$ at $Q^{2}<6 \mathrm{GeV}^{2}$ and was largely independent of the $\varepsilon$. The only contribution to $\mathcal{M}_{1}$ with a significant $\varepsilon$ dependence, and thus contributes to the form-factor discrepancy, is the two-photon exchange correction [37], which is given as,

$$
\delta^{2 \gamma} \equiv \frac{\operatorname{Re}\left\{\mathcal{M}_{0} \mathcal{M}^{2 \gamma}\right\}}{\left|\mathcal{M}_{0}\right|^{2}}
$$

where $\mathcal{M}^{2 \gamma}$ includes all the intermediate states of the proton. The total two-photon exchange amplitude is given as the sum of the contributions from the TPE box and 
crossed diagrams in Fig. 2.6 ((e) and (f) respectively) as,

$$
\mathcal{M}^{2 \gamma}=e^{4} \int \frac{d^{4} k}{(2 \pi)^{4}}\left[\frac{N_{b o x}}{D_{b o x}}+\frac{N_{\times-b o x}}{D_{\times-b o x}}\right],
$$

where $N_{b o x}$ and $N_{\times-b o x}$ are the respective matrix elements for box and crossed-box TPE diagrams. $D_{b o x}$ and $D_{\times-b o x}$ are the respective propagators [37].

Assuming the proton propagates as a Dirac particle, they considered only the elastic contribution to the full response function. Using phenomenological form factors at the $\gamma p$ vertices, they calculated the full IR divergent TPE contribution to the scattering amplitude from the box and crossed TPE diagrams [37]:

$$
\delta_{I R}=-2 \frac{\alpha}{\pi} \ln \left(\frac{E}{E^{\prime}}\right) \ln \left(\frac{Q^{2}}{\lambda^{2}}\right)
$$

where $\lambda$ is an infinitesimal photon mass required to regulate the IR divergences in the photon propagator. $E$ and $E^{\prime}$ are the energies of the incident and the scattered electron. The IR divergences occur when the four-momentum transfer carried by one photon is dominant over the four-momentum transfer carried by the other photon. Fig. 2.9 (left panel) shows the difference between IR divergent contributions from the BMT, and the standard Mo and Tsai (MT) calculations [29] plotted as a function of $\varepsilon$ at different $Q^{2}$. It is clear that the difference between two methods can lead to about a $1 \%$ change in cross section at the lowest $\varepsilon$ and only a minimal change at higher $\varepsilon$. This effect alone gives a reduction of the order of $3 \%$ and $7 \%$ at $Q^{2}=3 \mathrm{GeV}^{2}$ and $Q^{2}=7 \mathrm{GeV}^{2}$ in the form factor ratio $R=\frac{\mu_{p} G_{E}}{G_{M}}[37]$.

They also calculated the full TPE corrections $\left(\delta_{\text {full }}\right)$ including both the model dependent and the IR divergent terms, which contribute to the form factor discrepancy. In Figure 2.9 (right panel), the difference between the full TPE corrections and the IR divergent corrections is plotted as the function of $\varepsilon$ at different $Q^{2}$. The difference is 

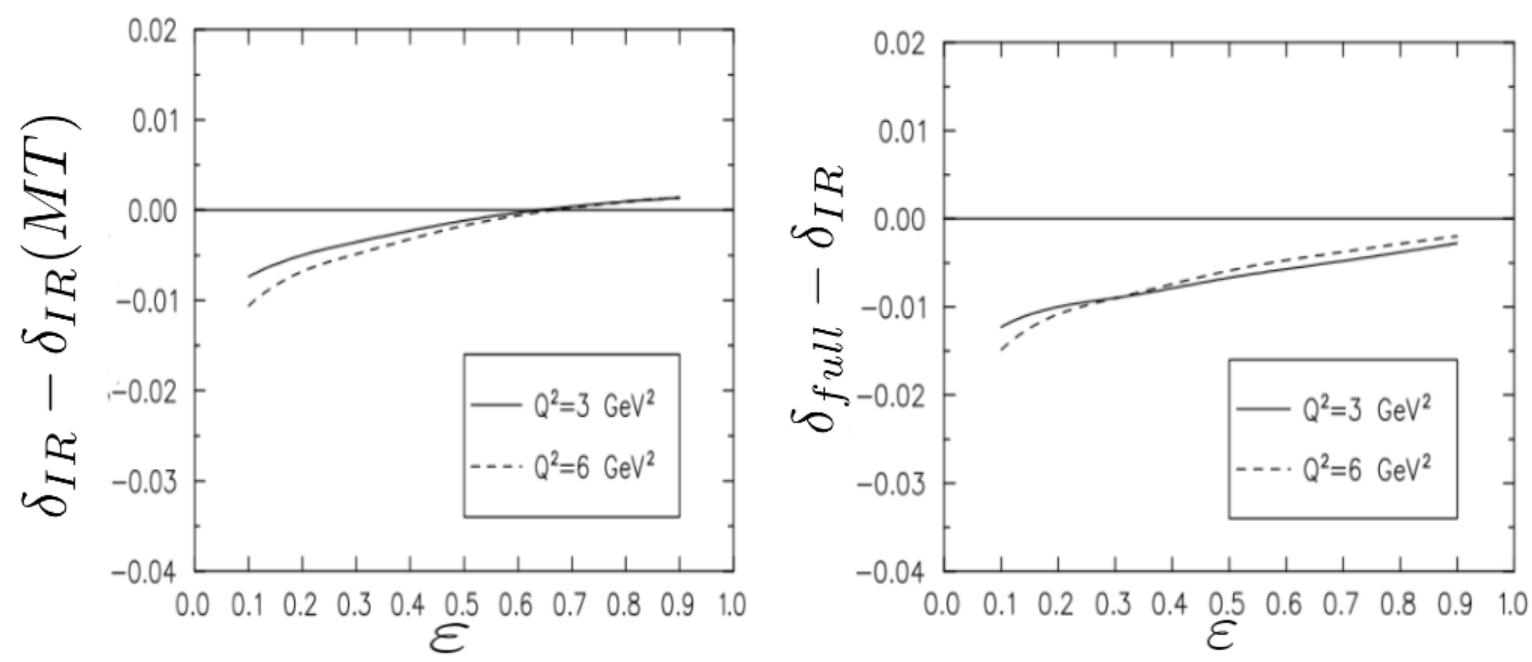

Figure 2.9: The $\varepsilon$ dependence of the difference between the model-independent and Mo and Tsai [29] IR divergent contributions at different $Q^{2}$ (left) and the difference between the full two-photon exchange correction and the the model independent IR divergence contributions at different $Q^{2}$ (right). Figure adopted from [37].

the largest at lower $\varepsilon$ and decreases at higher $\varepsilon$. The difference between the left and the right panel in Fig. 2.9 gives $\Delta=\delta_{f u l l}-\delta_{M T}$. The IR contributions cancel in the difference leaving only the contribution from the finite part of the difference. Hence, the finite part of the TPE amplitude shows a significant $\varepsilon$ dependence that slightly increases with $Q^{2}$.

They calculated the effect on the ratio $R$ in the Rosenbluth data assuming the modified cross section is approximately linear in $\varepsilon$ and has the form

$$
\sigma=(a A) \tau G_{M}\left(Q^{2}\right)\left[a+\left(B \tilde{R}^{2}+b\right) \varepsilon\right]
$$

where $B=1 / \mu_{p}^{2} \tau, \tilde{R}$ is the corrected ratio, $a$ and $b$ are the parameters of the linear fit function, and $A$ is a constant. The corrected ratio then becomes,

$$
\tilde{R}^{2}=R^{2}-b / B
$$




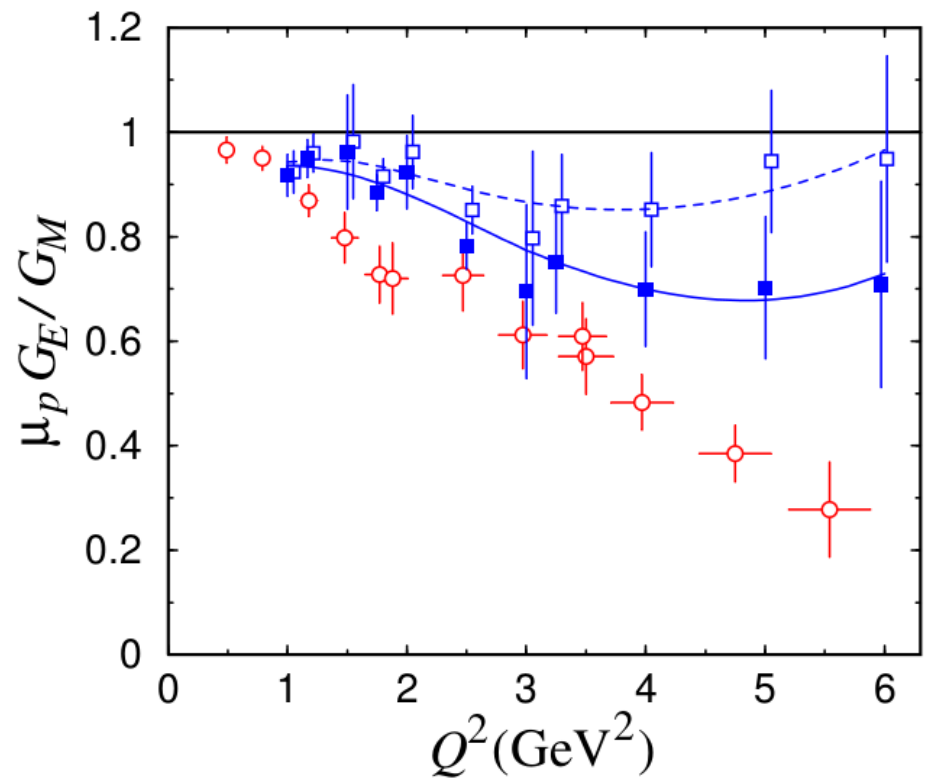

Figure 2.10: The form factor ratio measured by the Rosenbluth method(hollow squares) and polarization transfer method (hollow circles) as the function of $Q^{2}$. The filled squares are the BMT corrected Rosenbluth data. The dotted and the solid lines represent the global fit to the uncorrected and the BMT corrected data. Figure adopted from Ref. [37].

As shown in Fig. 2.10, the corrected ratio $R$ significantly brings down the Rosenbluth results towards the polarization transfer results. Using the hadronic model, the BMT calculation demonstrated that the two photon exchange correction has the sign and magnitude required to partially resolve the form factor discrepancy. In addition to these nucleon contributions, the contributions from the $\Delta$ and other heavier resonances were also added to the TPE calculation [38, 39]. These additions improve the agreement between the Rosenbluth and polarization transfer results.

\subsubsection{Generalized Parton Distribution (GPD) Model}

In the GPD model, Afanasev et.al calculated the TPE amplitude by relating it to generalized parton distributions [33]. Assuming the electron scatters from quarks inside the proton, they calculated the two-photon exchange part of the scattering 


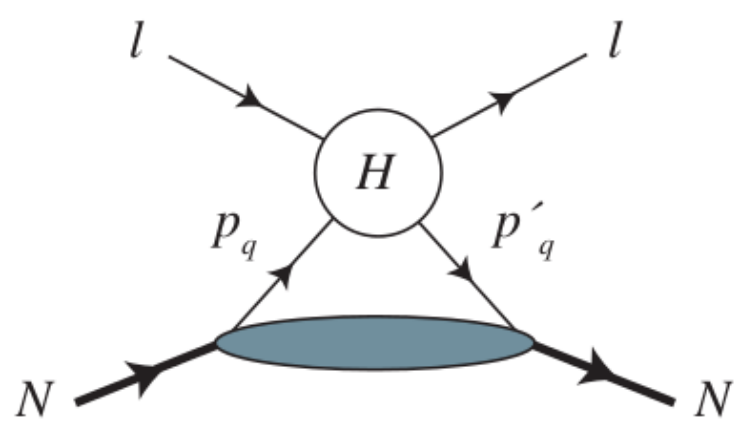

Figure 2.11: The handbag diagram for elastic lepton-quark scattering model. Figure adopted from 33 ]

amplitude for the partonic subprocess. The real and the imaginary part of the hard $\left(Q^{2}>>M^{2}\right)$ and the soft part of the amplitude were separately calculated. At the partonic level, the TPE correction term to the electron-quark elastic cross section was calculated as [33]:

$$
\begin{aligned}
\delta_{2 \gamma} & =\frac{e^{2}}{4 \pi^{2}}\left\{2 \ln \left(\frac{\lambda^{2}}{Q^{2}}\right) \ln \left|\frac{\hat{s}}{\hat{u}}\right|+\frac{(\hat{s}-\hat{u}) Q^{2}}{2\left(\hat{s}^{2}+\hat{u}^{2}\right)}\left[\ln ^{2}\left|\frac{\hat{s}}{Q^{2}}\right|+\ln ^{2}\left|\frac{\hat{u}}{Q^{2}}\right|+\pi^{2}\right]\right. \\
& \left.+\frac{Q^{4}}{\hat{s}^{2}+\hat{u}^{2}}+\left[\frac{\hat{u}}{Q^{2}} \ln \left|\frac{\hat{s}}{Q^{2}}\right|-\frac{\hat{s}}{Q^{2}} \ln \left|\frac{\hat{u}}{Q^{2}}\right|\right]\right\}
\end{aligned}
$$

where $\hat{s}=\left(k+p_{q}\right)^{2}$ and $\hat{u}=\left(k-p_{q}^{\prime}\right) 2$ are Lorentz invariant Mandelstam variables for the handbag diagram in Fig. 2.11. Then the partonic handbag formalism was extended to calculate the hard two-photon exchange contribution using a Gaussianvalence model and modified a Regge model for the GPDs. It is clear from Fig. 2.12 that the correction brings the Rosenbluth data into better agreement with the polarization data at $Q^{2}$ up to $2-3 \mathrm{GeV}^{2}$. However, at higher $Q^{2}$, the correction only partially reconciles the discrepancy. 


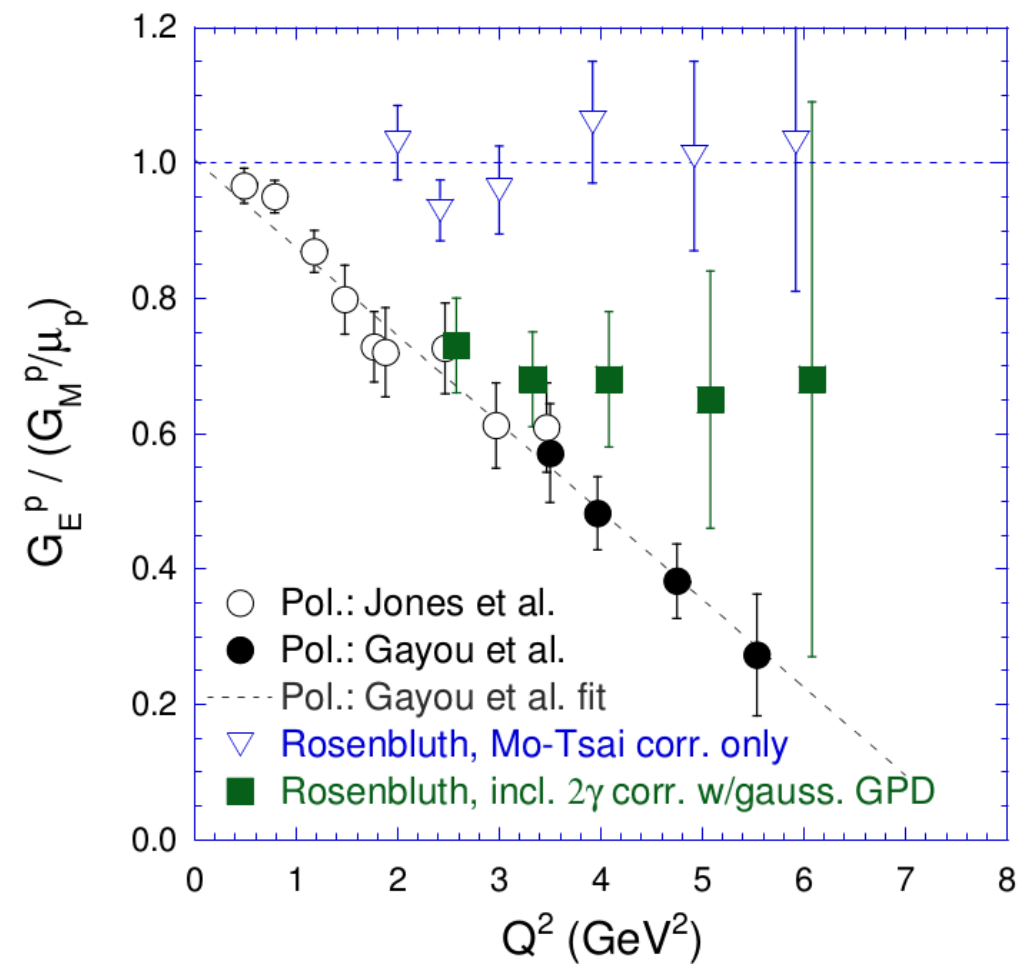

Figure 2.12: Form factor ratio before and after applying partonic TPE corrections to Rosenbluth data. The Rosenbluth data (hollow triangles) are from Ref. 9] and the polarization transfer data are from Ref. [12, 14]. The filled squares are the Rosenbluth data after partonic TPE corrections. Figure adopted from Ref. 33]

\subsubsection{Summary of the Theoretical Calculations of TPE}

In addition to the theoretical models discussed above, several other theoretical calculations yield TPE contributions that can resolve a large part of the discrepancy [40, 41, 42, 43, 44, 45, 46]. The details of these calculations and related issues can be found in recent review papers [30, 47]. A model independent measurement of the TPE effect can be obtained by the ratio of positron-proton elastic scattering cross section to that of the electron-proton. The BMT calculation, based on the hadronic model, predicts that $e^{+} p$ to $e^{-} p$ cross section ratio is larger than 1 at lower $\varepsilon$ and falls close to 1 at higher $\varepsilon$ [37. However, the calculations that use GPDs predict that the ratio is suppressed below one at large $\varepsilon$ and moderate $Q^{2}$ [33, 48. However, at low $\varepsilon$ and $Q^{2}$ 
of a few $\mathrm{GeV}^{2}$, the ratio is predicted to differ from one by as much as $10-15 \%$ [41]. Hence, to constrain the theoretical models and to make a definitive test of the TPE mechanism, a significantly precise measurement of the ratio of positron-proton elastic scattering cross section to that of electron-proton is required.

\subsection{Experimental Measurement of Two-Photon Exchange Ef- fect}

From QED, the total amplitude for elastic lepton-proton scattering can be written as [49],

$$
\mathcal{M}_{\text {total }}=q_{l} q_{p}\left[\mathcal{M}_{1 \gamma}+q_{l}^{2} \mathcal{M}_{\text {l.vertex }}+q_{p}^{2} \mathcal{M}_{\text {p.vertex }}+q_{l}^{2} \mathcal{M}_{\text {loop }}+q_{l} q_{p} \mathcal{M}_{2 \gamma}\right]
$$

where $q_{l}$ and $q_{p}$ are the charge of the lepton and proton, $\mathcal{M}_{1 \gamma}$ represents the Born amplitude, $\mathcal{M}_{\text {l.vertex }}$ and $\mathcal{M}_{\text {p.vertex }}$ describe the lepton and proton vertex corrections, $\mathcal{M}_{\text {loop }}$ describes the loop corrections and $\mathcal{M}_{2 \gamma}$ represents the two-photon corrections. Note that the contributions from the lepton and proton bremsstrahlung terms have been left out in the above equation. Squaring the total amplitude and keeping the terms up to the order $\alpha$, we get,

$$
\left|\mathcal{M}_{\text {total }}\right|^{2} \simeq e^{4}\left[\mathcal{M}_{1 \gamma}^{2}+2 e^{2} \mathcal{M}_{1 \gamma} \operatorname{Re}\left(\mathcal{M}_{\text {loop }+ \text { vertex }}\right)+2 q_{l} q_{p} \mathcal{M}_{1 \gamma} \operatorname{Re}\left(\mathcal{M}_{2 \gamma}\right)\right]
$$

Here, $q_{l}^{2}$ and $q_{p}^{2}$ are replaced by $e^{2}$. We know the scattering cross section is proportional to the square of the amplitude $\left(\sigma \propto\left|\mathcal{M}_{\text {total }}\right|^{2}\right)$. Hence, the dominant contribution to the cross section comes from the Born term $\left(\mathcal{M}_{1 \gamma}\right)$ as it is real and larger than other terms in Eq. 2.62. The contribution from the imaginary part of $\mathcal{M}_{2 \gamma}$ being small and negligible, only the real part of $\mathcal{M}_{2 \gamma}$ is kept in Eq. 2.62. It is clear from Eq. 2.62 that only the interference between the one and two-photon exchange term depends on the sign of the lepton charge. 
It is necessary to include lepton and proton bremsstrahlung contributions in Eq. 2.62 as we cannot separate true elastic events $(e p)$ from events with a radiated photon in the final state $(e p \gamma)$. The lepton and proton bremsstrahlung terms add another lepton-charge sign-dependent term to the final cross section,

$$
\left|\mathcal{M}_{\text {total }}\right|^{2} \simeq e^{4}\left[\mathcal{M}_{1 \gamma}^{2}+2 e^{2}\left(C_{\text {even }}\right)+2 q_{l} q_{p}\left(\mathcal{M}_{1 \gamma} \operatorname{Re}\left(\mathcal{M}_{2 \gamma}\right)+\operatorname{Re}\left(\mathcal{M}_{\text {e.br. }}^{*} \mathcal{M}_{p . b r .}\right)\right]\right.
$$

where $C_{\text {even }}$ is the sum of the charge-even part of the radiative contributions, including both the loop and vertex diagrams and the charge-even contributions from the lepton and proton bremsstrahlung terms. The only charge odd terms are the interference between lepton and proton bremsstrahlung terms and the interference between one and two photon exchange terms. Eq. 2.63 can be expressed as the Born term plus additional corrections from the charge-even and charge-odd corrections i.e.

$$
\sigma=\sigma_{\text {born }}\left(1+\delta_{\text {even }}+q_{l} q_{p} \delta_{2 \gamma}+q_{l} q_{p} \delta_{e . p . b r .}\right)
$$

where $\delta_{\text {even }}=2 e^{2} C_{\text {even }} / \mathcal{M}_{1 \gamma}^{2}$ is the contribution from the charge-even terms, $\delta_{2 \gamma}$ is the the contribution from the interference between one and two photon exchange term, and $\delta_{\text {e.p.br. }}$ is the contribution from the lepton and proton bremsstrahlung interference term. The infrared divergences of both of these terms cancel in the sum of the two contributions. It is obvious that the last two terms of Eq. 2.64 depend upon the sign of the charge and thus contribute to the charge asymmetry in elastic $e^{ \pm} p$ scattering. Taking the ratio of $e^{+} p$ to $e^{-} p$ elastic scattering cross sections as:

$$
\begin{aligned}
\frac{\sigma\left(e^{+} p\right)}{\sigma\left(e^{-} p\right)} & \simeq \frac{1+\delta_{\text {even }}-\delta_{2 \gamma}-\delta_{\text {e.p.br. }}}{1+\delta_{\text {even }}+\delta_{2 \gamma}+\delta_{\text {e.p.br. }}} \\
& \simeq 1-\frac{2\left(\delta_{2 \gamma}+\delta_{\text {e.p.br. }}\right)}{\left(1+\delta_{\text {even }}\right)}
\end{aligned}
$$


Here, the sign of $\delta_{2 \gamma}$ and $\delta_{\text {e.p.br. }}$ are chosen in such a way that the corrections are additive for $e^{-} p$ scattering. The TPE effect can be isolated from the above equation if we apply a correction for $\delta_{\text {e.p.br. }}$. The $e p$ bremsstrahlung interference corrected ratio then becomes:

$$
R_{2 \gamma}=1-\frac{2 \delta_{2 \gamma}}{1+\delta_{\text {even }}} .
$$

It is to be noted that most of the previous extractions neglect the charge even contributions coming from the $\left(1+\delta_{\text {even }}\right)$ term in the denominator. $\delta_{\text {even }}$ being a small negative correction, $\left(1+\delta_{\text {even }}\right)$ in the denominator always becomes smaller than 1 . Hence, the ratio is reduced because of this charge-even correction and $R_{2 \gamma}$ is systematically underestimated by approximately $1 \%$. However, Eq. 2.66 shows that TPE effects can directly be measured by comparing the ratio cross sections for positronproton to electron-proton elastic scattering with proper radiative corrections for $\delta_{\text {e.p.br. }}$. and $\delta_{\text {even }}$.

\subsection{Existing Data for the Cross Section Ratio}

The $e^{+} p$ and $e^{-} p$ cross section ratio has been measured by several experiments to investigate the TPE effect. The measured cross section ratio from these experiments are shown in Fig. 2.13 as a function of $Q^{2}$ (left) and $\varepsilon$ (right).

As can be seen in the left panel of Fig. 2.13, there is no clear indication of a $Q^{2}$ dependence of the cross section ratio. The right panel of Fig. 2.13 indicates a slight $\varepsilon$ dependence of the cross section ratio. The linear fit to this $\varepsilon$ dependence yields $\approx 6 \%$ slope implying approximately a $3 \%$ contribution from TPE to the $e^{-} p$ elastic scattering cross section [30], which is about half of the size required to explain the discrepancy at higher $Q^{2}$. However, because of the limited statistics, the results are not very precise at low $\varepsilon$ and high $Q^{2}$ where the discrepancy is large. Hence, these data 

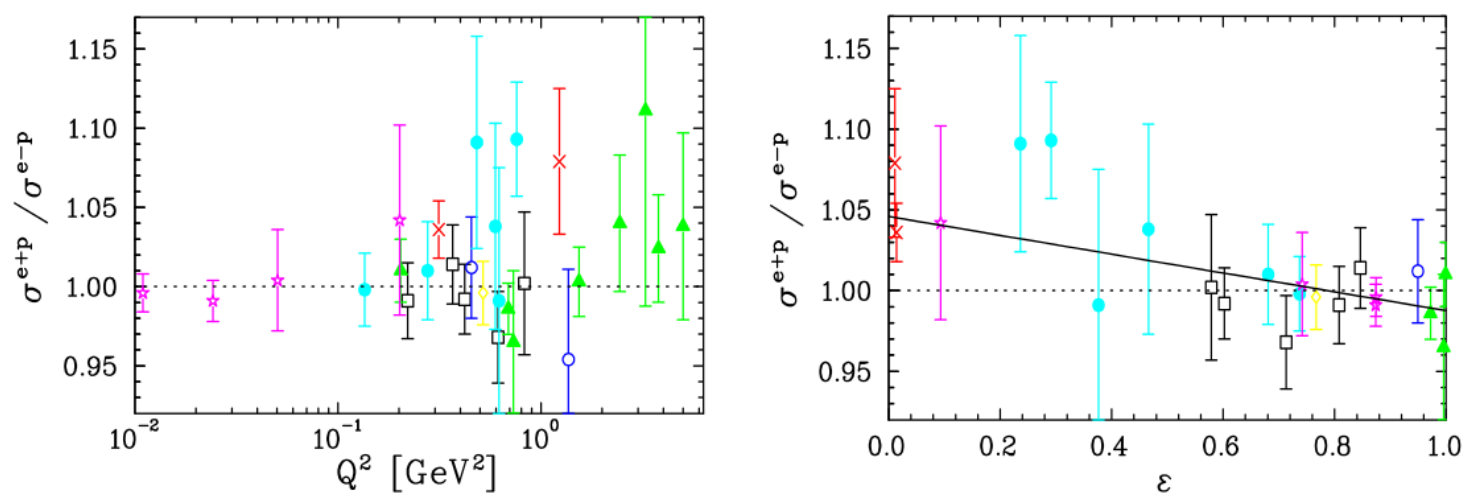

Figure 2.13: The ratio of $e^{+} p$ to $e^{-} p$ elastic scattering cross section as the function of $Q^{2}$ (left) and $\varepsilon$ (right). The solid line is a linear fit to the data. Figure adopted from Ref. 30]

are not sufficient to draw any strong conclusion about the size of the TPE effects. In light of this discrepancy, several collaborations have completed their data collection to measure the ratio in an effort to shed light on the form factor discrepancy [50, 51, 52]. The data analyses from the new generation of the TPE experiments are in progress in order to obtain the TPE effects by comparing the ratio of $e^{+} p$ to $e^{-} p$ elastic scattering cross sections. One of the experiments was conducted at the Hall B of the Jefferson Lab using CLAS. The focus of the remainder of this dissertation is the description of the CLAS TPE experiment, its data analysis procedure, and the results. 


\section{Chapter 3}

\section{Experimental Apparatus}

\subsection{Overview}

The TPE experiment collected data from December, 2010 through February, 2011 in experimental Hall B at Jefferson Lab, in Newport News, Virginia. A Mixed electron-positron beam was produced from the primary electron beam provided by the Continuous Electron Beam Accelerator Facility (CEBAF). The mixed beam was then incident on a liquid hydrogen target placed at the center of a $4 \pi$ hermetic spectrometer known as CEBAF Large Acceptance Spectrometer (CLAS). The scattered particles were then detected by CLAS. Unlike other CLAS experiments, this experiment required simultaneous and identical electron-positron beams. Thus, several modifications to the existing Hall B beamline were required for this experiment. This Chapter discusses the experimental setup in detail, including the accelerator facility, CLAS detector components, and the TPE experimental beamline modifications.

\subsection{CEBAF}

CEBAF at the Jefferson Lab is a continuous wave, superconducting radio frequency (SRF) accelerator. The accelerator consists of two Linear Accelerators (LinAcs) connected by two re-circulating arcs to form a $1.4 \mathrm{~km}$ underground racetrack [53]. A schematic diagram of the accelerator's racetrack layout is shown in Fig. 3.1. Each LinAc contains twenty cryomodules, each containing eight SRF cavities made of Niobium [53]. Niobium is a superconductor below its transition temperature $(\approx 9 \mathrm{~K})$ and is widely used in SRF technologies.

CEBAF can simultaneously deliver $499 \mathrm{MHz}$ electron beams to the three experimen-

tal halls A, B and C. The electron injector produces three interlaced electron beams 


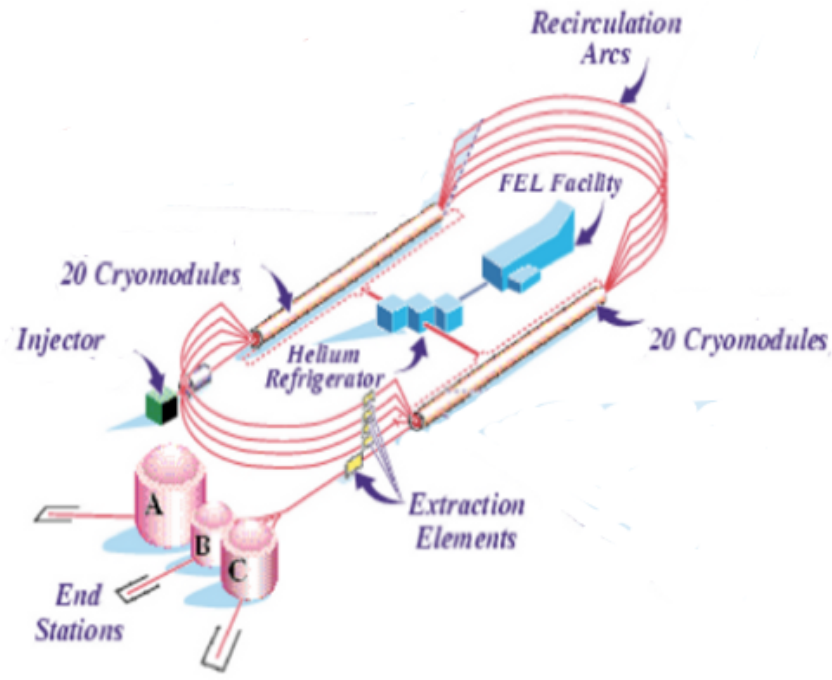

Figure 3.1: Schematic design of Jefferson lab accelerator

by optically illuminating a Gallium Arsenide (GaAs) photocathode [53]. The electron beams are then accelerated around the racetrack where they gain energy up to 1.125 $\mathrm{GeV}$ in each circulation. At the end of the second LinAc the beam can either be extracted to the experimental halls or further steered through a second $180^{\circ}$ recirculation arc for further acceleration. The beams can be recirculated up to five times, to a maximum energy of $5.625 \mathrm{GeV}$. In every $2.004 \mathrm{~ns}$, the electron bunches are delivered to an experimental hall. Jefferson Lab is currently upgrading its accelerator and other facilities to attain a maximum electron beam energy of $12 \mathrm{GeV}$ [54]. Another experimental hall, Hall D is also being constructed.

\subsection{Hall B and CLAS Detector}

Experimental Hall B houses a spherical, large-acceptance spectrometer, CLAS, at the center of the hall and a photon tagging system, which produces real photons by 
Bremsstrahlung radiation!. CLAS is a multi-layered spherical detector with nearly $4 \pi$ acceptance. It is divided into six sectors by superconducting coils that produce a toroidal magnetic field. The magnetic field bends the scattered particles towards (inbenders) or away (outbenders) from the beamline depending upon its polarity. Each sector of CLAS consists of three regions of drift chambers (DC) to determine charge particle trajectories, a Cerenkov counter (CC) to separate electrons from pions, an electromagnetic calorimeter (EC) for energy measurements of charged and neutral particles, and time-of-flight (TOF) counters for timing measurements. The CC and EC cover only the forward region of CLAS $\left(8^{\circ}<\theta<45^{\circ}\right)$. Fig. 3.2 shows an exploded view showing different elements of CLAS. Each sector of CLAS serves as an independent spectrometer with a common target, trigger, and data acquisition (DAQ) system [55]. The CLAS experiments generally use data from the CC and EC for particle identification purposes. However, this experiment did not use either the $\mathrm{CC}$ or the EC. The $\mathrm{CC}$ was not used because it is optimized for inbending particles. It would have different responses for inbenders versus outbenders and would create an asymmetry in particle detection. Similarly, the EC was not used because it wasn't necessary for particle identification and energy measurements for this experiment. It could only reduce the acceptance and add another layer of possible charge asymmetry in particle detection. In the following sections, each of the components of CLAS, which were used in this experiment, will be described in detail.

\subsubsection{Torus Magnet}

CLAS is constructed around six superconducting, kidney-shaped coils. Each coil consists of four layers of 54 turns of $\mathrm{NbTi} / \mathrm{Cu}$ conductor [55]. Liquid helium is

\footnotetext{
${ }^{1}$ The electromagnetic radiation produced by decelerating energetic electrons in the presence of the nucleus of a high atomic mass atom
} 


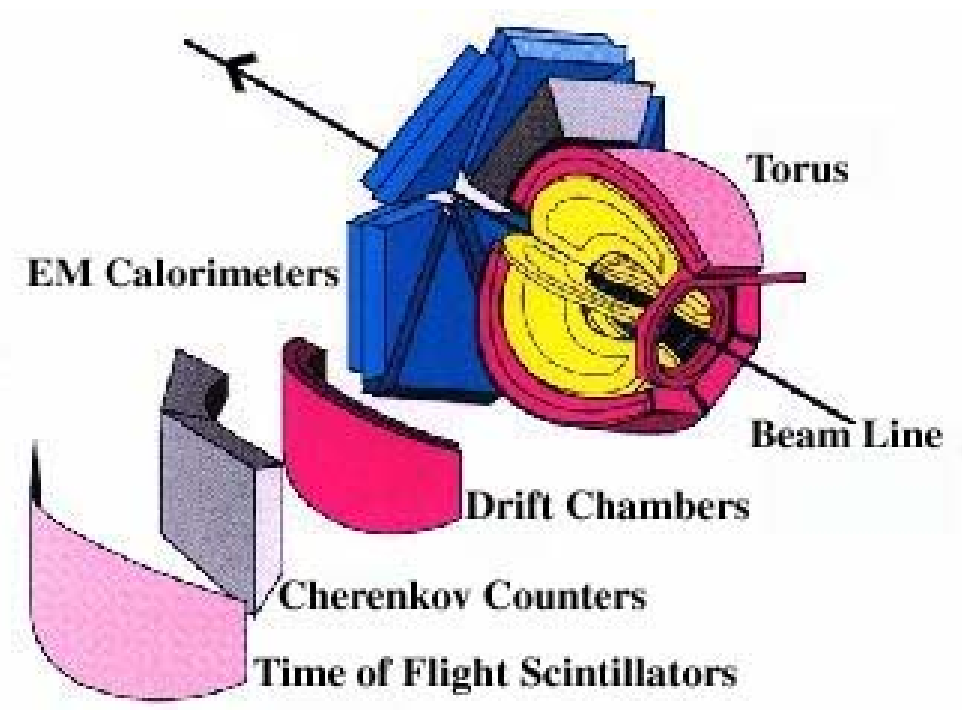

Figure 3.2: An exploded view of CLAS. Torus magnet (yellow), electromagnetic calorimeter (blue), drift chambers (light red), Čerenkov Counters, and the TOF scintillators (pink) are clearly visible. The mini-torus magnet is not visible. The straight line is along direction of the beam from lower right to upper left.

circulated around the coils through the cooling tubes to keep them at $4.5 \mathrm{~K}$. The coils generate a toroidal magnetic field around the beamline. Fig. 3.3 shows the contours of the magnetic field produced by the CLAS torus magnet. The field is maximum at small polar angles. The toroidal magnetic field bends particles towards or away from the beamline depending upon the charge of the particle. For positive (negative) torus, the negatively charged particles are bent towards (away from) the beamline, while the positively charged particles are bent away from (towards) the beamline. The momentum of the charged particles are reconstructed from their curvatures in the magnetic field. At the designed maximum current (3860 A), the integral of magnetic field $\left(\int \mathbf{B} \cdot \mathbf{d} \mathbf{l}\right)$ reaches up to $2.5 \mathrm{~T} \cdot \mathrm{m}$ in the forward region, but drops to $0.6 \mathrm{~T} \cdot \mathrm{m}$ at a scattering angle of $90^{\circ}$ [55]. CLAS routinely operates at a maximum current of 3375 A to keep the internal mechanical stresses of the torus magnets at a safe level [55]. During the TPE run period the torus magnet current was at $\pm 1500 \mathrm{~A}$. 


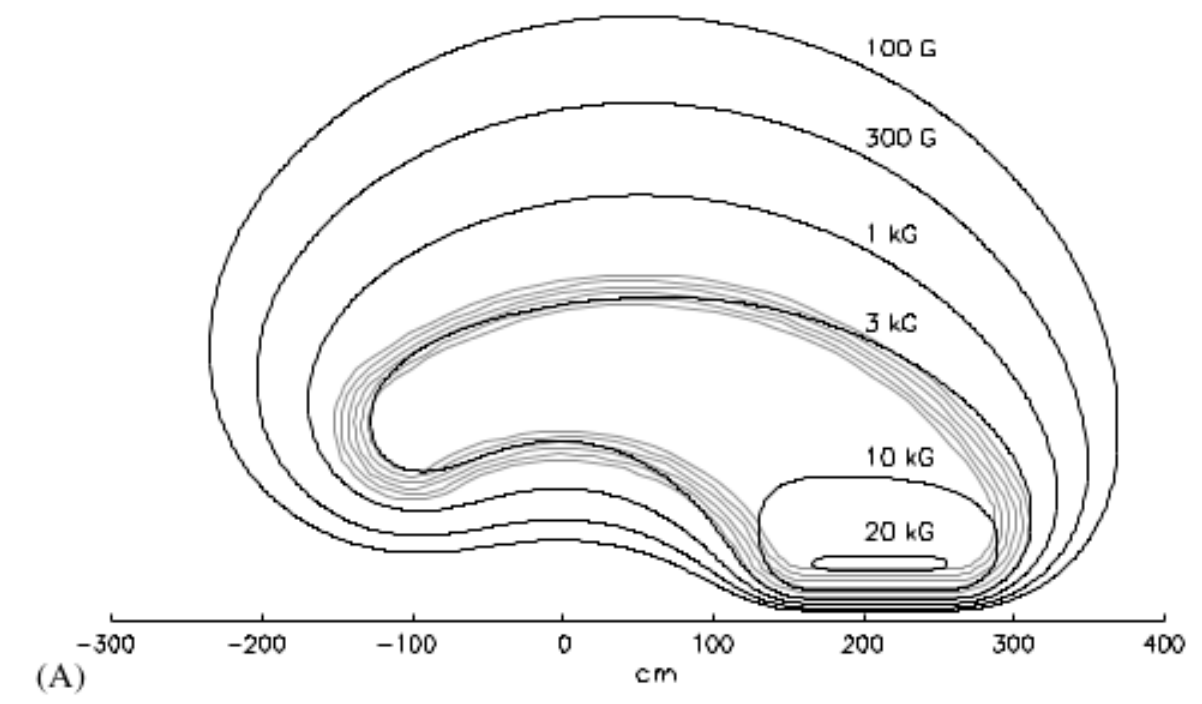

(A)

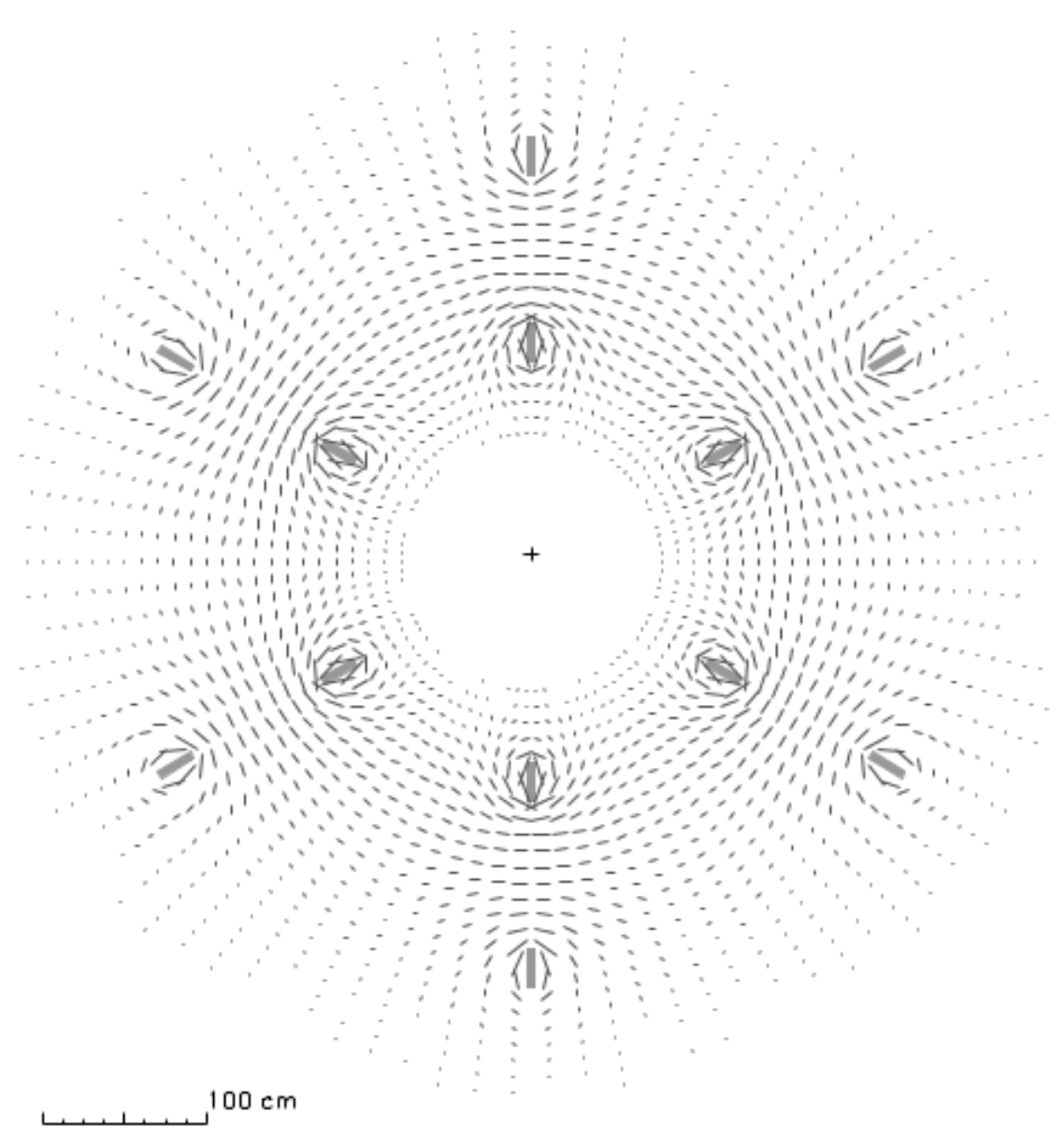

(B)

Figure 3.3: (A) Contours of the magnetic field produced by the CLAS torus in the mid-plane between two coils (B) Magnetic field vectors for the toroid transverse to the beam in a plane centered on the target 


\subsubsection{Mini-Torus Magnet}

In addition to the main torus magnet, this experiment utilized a non-superconducting mini-torus magnet, which surrounded the target. The mini-torus prevents low momentum particles from reaching the innermost drift chambers by bending them towards the beamline. These low momentum particles are produced via Møller scattering in the target during electron beam experiments so the mini-torus is used only during electron beam experiments. The current of the mini-torus magnet during the TPE production run was fixed at $4000 \mathrm{~A}$.

\subsubsection{Drift Chamber (DC)}

The drift chambers in CLAS are used for tracking of charged particles and reconstruction of their momentum as they traverse through the CLAS toroidal magnetic field. The schematics of the CLAS DC system is shown in Fig. 3.4. The CLAS drift chamber system consists of 18 separate drift chambers located at three radial locations in each sector. These radial locations are known as "Regions". The "Region One" (R1) chambers are in the field-free region of CLAS and surround the target. They provide initial direction of the charged-particle track. The "Region Two" (R2) chambers are located between the torus coils in the high magnetic field. The track curvature is maximum in this region. The "Region Three" (R3) chambers are located just outside the torus coils. They provide the final direction of the track as they emerge out of the toroidal magnetic field. All three regions of the drift chambers share the same basic design elements. Wires are strung between two end plates, each parallel to its neighboring coil plane. To keep the wire direction approximately perpendicular to the bend-plane of the curved trajectories, the end-plates are tilted at $60^{\circ}$ with respect to each other. This configuration provides maximum sensitivity to the track 


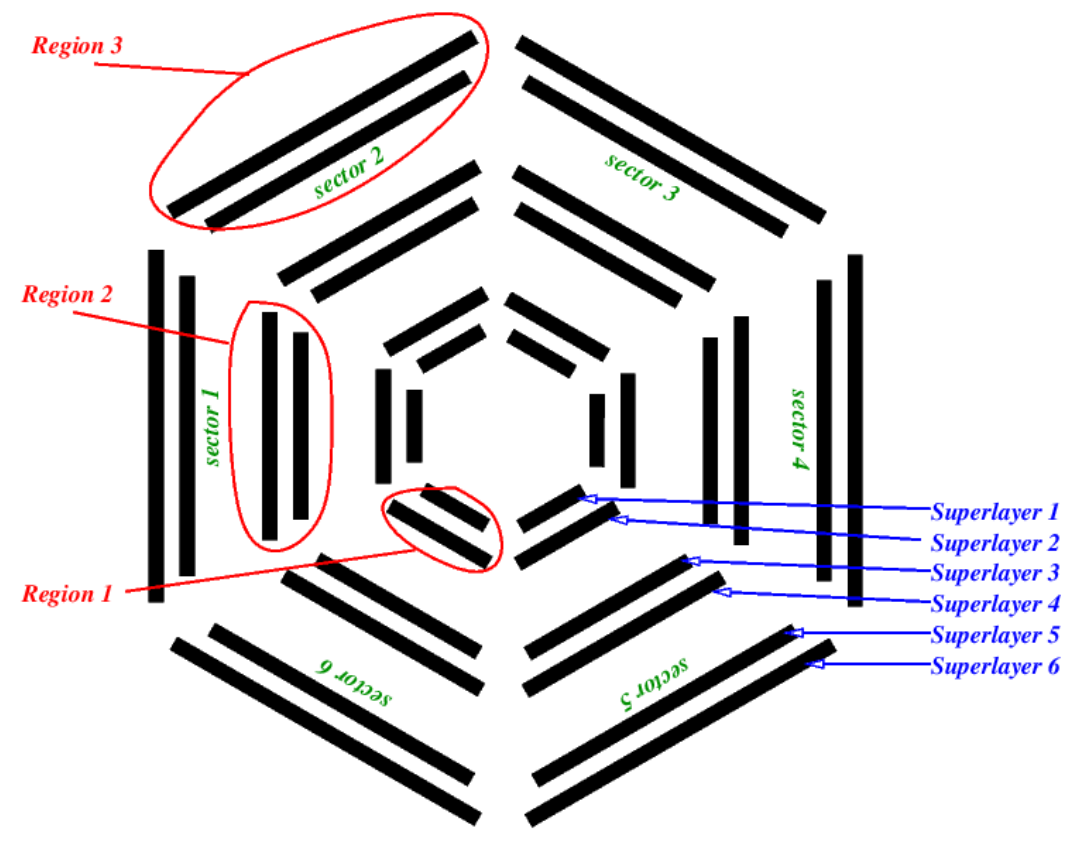

Figure 3.4: A vertical cut of the CLAS drift chambers through the target position. All the superlayers are shown.

curvature [56]. In order to improve the tracking resolution, the wire layers in each region are grouped into two "superlayers". One super layer is axial to the magnetic field and the other is at a $6^{\circ}$ stereo angle to provide azimuthal-angle information [56]. Each super-layer consists of six layers of drift cells. Each sense wire is surrounded by six field wires forming a hexagonal beehive structure as shown in Fig.3.5. In CLAS, the charged particles enter the chamber at widely varying angles. Ideally, the circular drift cells are desired because the drift time to drift distance relation in the cell is independent of the incident angle of the track. The hexagonal drift cells are a very close approximation to circular cells.

Each sense wire is a $20 \mu$ m-diameter gold-plated tungsten, while each field wire is a $140 \mu \mathrm{m}$-diameter gold-plated aluminum. The sense and the field wires are kept at positive and negative potential, respectively, by a high voltage system. Each of the 18 chambers is filled with a mixture of $90 \%$ Argon and $10 \% \mathrm{CO}_{2}$ [56]. When 


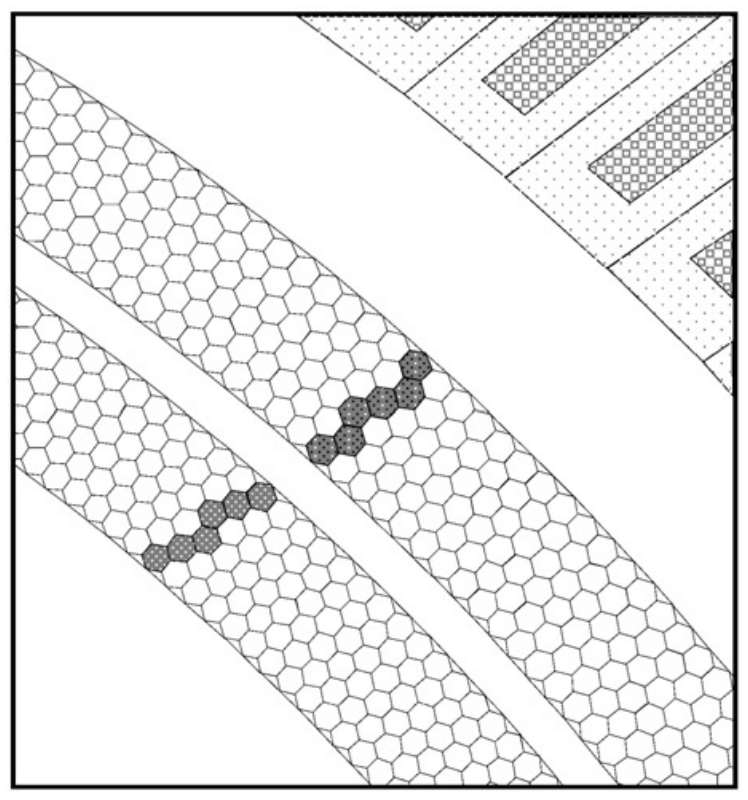

Figure 3.5: A portion of region 3 drift chamber showing hexagonal drift cells. The sense wires are at the center of the hexagon and field wires are at the vertices. The highlighted drift cells represent a charged-particle track. The Čerenkov counter is also visible on the top right corner.

traversing through the drift chamber volume, a charged particle ionizes the chamber gas into electron-ion pairs. The ionized electron drifts towards the sense wire with an approximately constant drift velocity. Near the sense wire, a high electric field causes the drifting electron to create multiple electron-ion pairs to produce a detectable signal on the sense wire. The signal is readout by a single-channel differential pre-amplifier mounted on a printed circuit board (PCB) attached to the chamber endplate. The outputs from each group of pre-amplifiers are sent to amplifier and discriminator board (ADB) to digitize the output pulses. The digitized output pulses are then transfered to TDC $\$$ to determine signal timing information, which is used to determine drift distances as described in Sect. 3.9.1 


\subsubsection{Time of Flight (TOF) Counters}

The CLAS TOF system is used to measure the time of flight of a charged particle from target to the TOF counters. The measured timing information is used for triggering purposes, and determination of particle speed, which is used in conjunction with the momentum information for particle identification. The TOF system in each CLAS sector consists of 57 scintillating paddles made up of Bicron BC-408 plastic, which has a fast response time. The thickness of each paddle is $5.08 \mathrm{~cm}$ and has a length that varies from 32 to $445 \mathrm{~cm}$. The angular coverage of the scintillators ranges from $8^{\circ}$ to $142^{\circ}$ in polar angle $(\theta)$ and almost covers the entire range in the azimuthal angle $(\phi)$. The scintillator paddles numbered 1 through 23 are mounted together in "panel 1" and are identified as "forward angle" counters. Panel 1 corresponds to the polar angles from $8^{\circ}$ to $45^{\circ}$. Paddles 24 though 34 are mounted in "panel 2", paddles 35 through 45 are mounted in "panel 3", and paddles 46 through 57 are mounted in "panel 4". Panels 2, 3 and 4 are referred as the "large angle" counters and correspond to the polar angles greater than $45^{\circ}$. Each scintillator paddle is coupled to a PMT at each end. In order to reduce the number of electronic channels, the last 18 scintillator paddles are grouped together electronically in pairs and are treated as single, double width paddles [57]. The width of each forward angle paddle is $15 \mathrm{~cm}$ and the width of the large angle paddles is $22 \mathrm{~cm}$.

As a charged particle passes through the scintillator, the atoms in the scintillator material become excited. The atomic excitation results in the emission of photons, which are then directed towards the photomultiplier tube (PMT) through the light guides via total internal reflection. The photons are incident on the photocathode of the PMTs and release electrons through the photoelectric effect. The resulting electrons then cause multiple secondary electrons to be emitted in a series of accelerating 


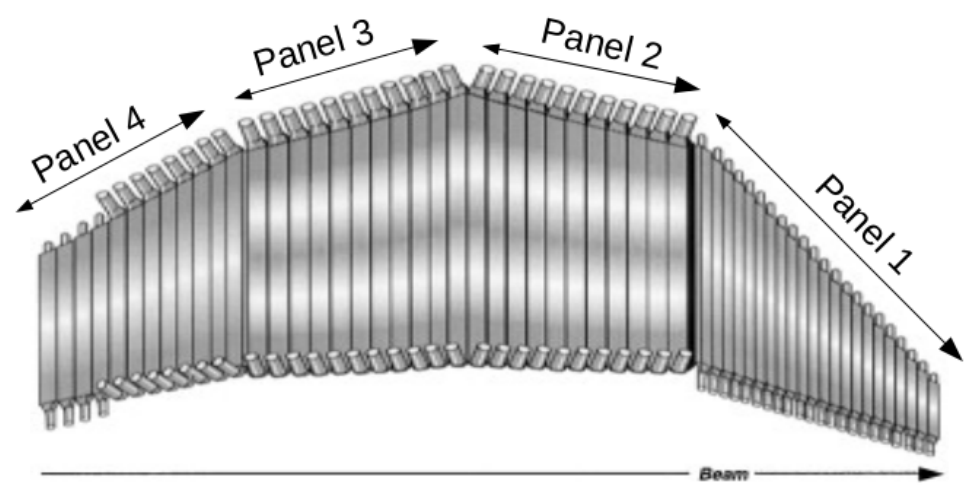

Figure 3.6: View of TOF counters in one of CLAS sectors showing the grouping into four panels. The beam direction is shown by the arrow.

electrodes, called dynodes, in the PMTs. This electron multiplication chain inside the PMT converts the optical light signal to a detectable electric pulse with a large amplification. These pulses generated by the TOF counters are then used for CLAS trigger electronics as well as for pulse-height and timing analysis [57].

The TOF trigger is set when a track passes through the scintillator with deposited energy greater than some pre-selected threshold value. A pre-trigger circuit divides the PMT dynode pulses into two signals, one of which is sent to the CLAS Level I trigger, and the other one is used for pulse height and timing analysis. The time of the event is determined by LeCroy 1872A FASTBUS TDC triggered by a LeCroy 2313 discriminator set at low threshold for precise timing [57].

\subsubsection{Electromagnetic Calorimeter (EC)}

The forward electromagnetic calorimeter in CLAS covers the polar angle in the range $8^{\circ}<\theta<45^{\circ}$. In normal CLAS operation, the EC is used for triggering, electron pion separation, neutral particle identification, and total energy measurements. For this experiment, the EC was used only to trigger the data acquisition system as 


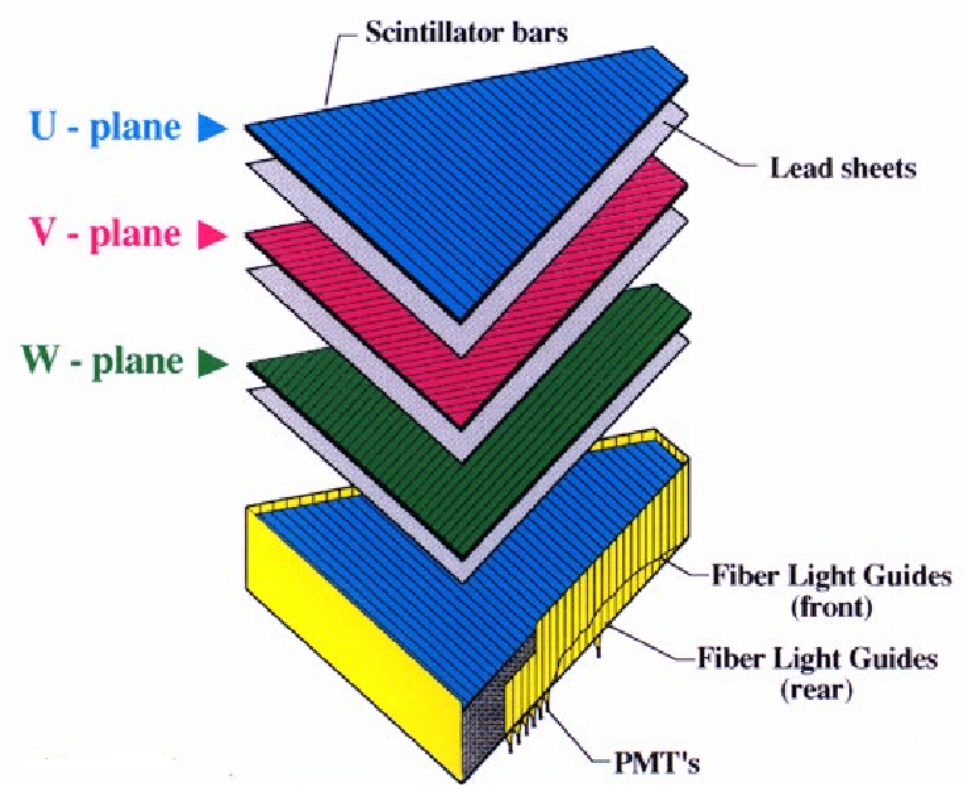

Figure 3.7: Exploded view of the Electromagnetic Calorimeter module in a CLAS sector. The $\mathrm{U}, \mathrm{V}$, and $\mathrm{W}$ planes are clearly visible.

discussed in Sect. 3.6. The EC modules in each sector are located just outside the TOF counters, in the forward region of CLAS. Each EC module has the shape of an equilateral triangle and consists of alternating layers of 2.2-mm-thick lead sheets, and 10-mm-thick strips of plastic scintillator. The total thickness of each module is 16 radiation-lengths ${ }^{2}(R L)$. Each scintillator layer is made up of 36 strips, parallel to one side of the equilateral triangle. The strips in each successive layer are rotated by $120^{\circ}$ forming three orientations that are labeled as $\mathrm{U}, \mathrm{V}$, and $\mathrm{W}$. These orientations provide stereo location of energy deposition. Each orientation contains 13 layers of a lead-scintillator sandwich, which are subdivided into an inner stack (5 layers) and the outer stack (8 layers). These stacks provide longitudinal sampling of the shower for improved electron/hadron separation [58]. The exploded view of one of the EC

\footnotetext{
${ }^{2}$ The characteristics length of matter related to the energy lost by a high-energy particle when traversing through it and is usually measured in $\mathrm{g} \mathrm{cm}^{-2}$. It is: a) the mean distance over which a high-energy electron loses all but 1 /e of its energy by bremsstrahlung b) $7 / 9$ of the mean free path for pair production by a high-energy photon [18]
} 


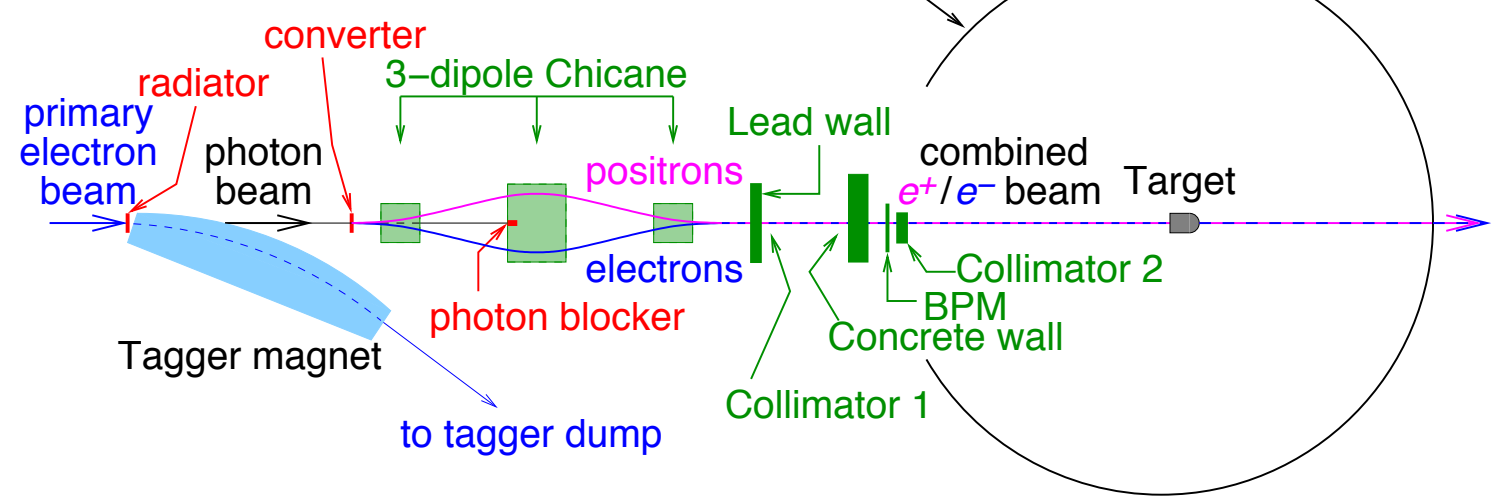

Figure 3.8: Schematic diagram of the TPE beamline

module is shown in Fig. 3.7. The signal through each module is read out by using 36 (strips) $\times 3$ (orientations) $\times 2$ (stacks $)=216$ PMTs. The scintillator light is transmitted to the PMTs by using fiber-optic light-readout units. All 1296 PMT channels are read out with LeCroy 1881M ADC and LeCroy 1872 TDC boards. The timing signals to the TDCs are provided by LeCroy 2313 leading edge discriminators. The details of the electronics system used in EC can be found in Ref. [58].

\subsection{TPE Beamline Components}

To produce a simultaneous mixed electron-positron beam required for the experiment, several modifications to the existing Hall B beamline were required. Modified components of the beamline included a converter, a series of dipole magnets known as the "chicane", beam profile monitors and a beam profile calorimeter. A schematic diagram of the beamline components of the TPE experiment is shown in Fig. 3.8. Bremsstrahlung photons were produced by striking a gold radiator with a 100- to 120-nA 5.6-GeV primary electron beam provided by the CEBAF accelerator. The electrons were diverted into the Hall B tagger dump by using the tagger magnet. The resulting photon beam then passed through a 12.7-mm-diameter nickel collimator and struck a 0.09 RL gold converter to produce electron-positron pairs. The mixed electron, positron, and photon beam then entered the 3-dipole chicane that separated 


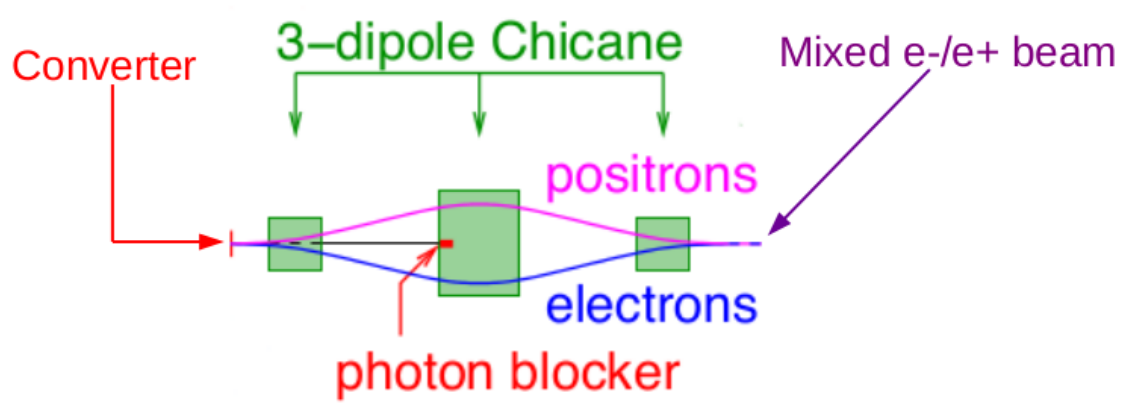

Figure 3.9: Schematic diagram of the three dipole chicane

electron and positron in the horizontal plane and stopped the photon. This section will discuss the modified beamline components in detail.

\subsubsection{Chicane}

A series of three dipole magnets known as the chicane, was employed to separate the photon beam from the mixed lepton beam. Fig. 3.9 shows the schematic diagram of the TPE chicane. The first and the third dipoles in this setting were the so-called Italian dipoles (ID), and the second magnet was the pair spectrometer (PS). As the mixed beam entered the first dipole, the electron and positron beams separated spatially in the horizontal plane since they bend in the opposite directions in a given magnetic field. The photon beam continued to traverse along the beamline, and was stopped by a 4-cm-wide and 35-cm-long tungsten photon blocker placed at the upstream face of the second dipole. The magnetic field was set in such a way that the electron and positron beams converge as they emerge out of the second magnet. A mixed electron-positron beam emerged out of the third dipole, and traversed along the beamline. In the reversed chicane polarity, the electron and positron beams switch their spatial positions. Ideally, there would not be any left-right spatial asymmetry between electron and positron beams as the three dipoles are left-right symmetric. 
Even if the chicane was not perfectly symmetric, periodically reversing the chicane polarities should cancel any spatial asymmetries. This is discussed further in Sect. 4.5 and Sect. 5.2.3.

Either of the diverging lepton beams could be stopped at the exit face of the first dipole by inserting one of the two "beam blockers" in their path. These lepton beam blockers are standard-sized lead bricks $(2 " \times 4 " \times 8$ ") and were used to block individual lepton beams during beam position measurements and chicane-magnetic field optimization. The lepton beam blockers were not used during normal production data taking.

\subsubsection{Sparse Fiber Monitor}

The position of the mixed beam was monitored throughout the experiment using a scintillating fiber monitor, hereby referred to as the sparse fiber monitor (SFM). This device was designed and built for the previous version of this experiment [59] and was rebuilt by the author at FIU for this experiment. The schematic diagram for the device is shown in Fig. 3.10. It contains 32, $1 \times 1 \mathrm{~mm}$ multiclad Bicron (BCF-12) fibers, supported by $6 " \times 6$ " aluminum frame. 16 fibers are laid along the $\mathrm{x}$-axis parallel to each other, while the other 16 fibers are laid along the y-axis, parallel to each other, creating a grid mesh structure for two-dimensional readout. Consecutive fibers are $5 \mathrm{~mm}$ apart. Each set of 16 fibers are optically glued to a multi-pixel Hamamatsu PMT for light collection and amplification.

The SFM was installed upstream of the TPE target to monitor the position of the beam. When the beam traverses through the SFM, the scintillation light emitted by the fibers is collected by two PMTs, which provide SFM coordinates of the beam. Fig. 3.11 shows typical beam distributions along horizontal (x) and vertical (y) fibers 


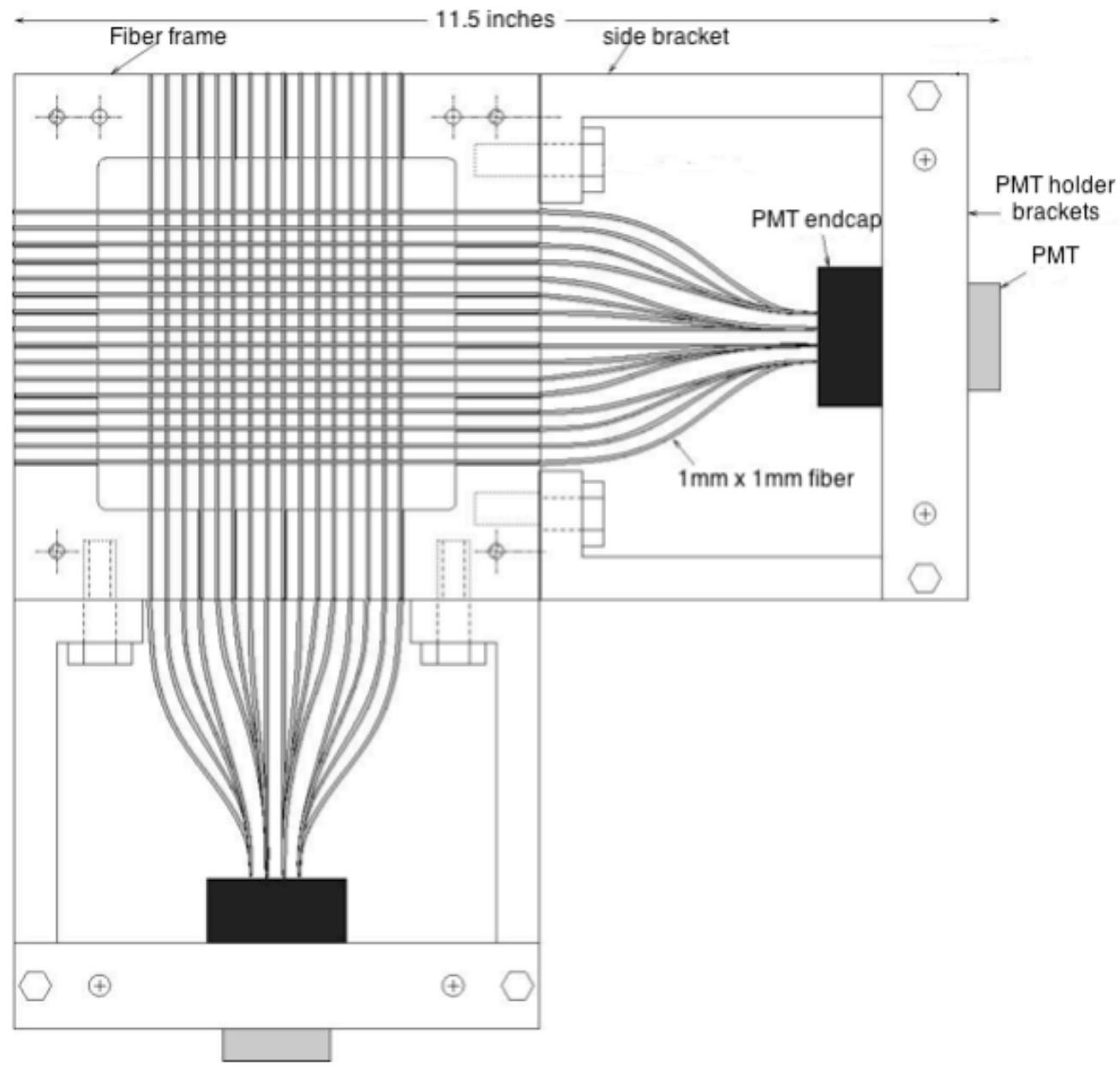

Figure 3.10: Schematic diagram of the TPE sparse fiber monitor. The beam direction is into the plane.

at the SFM during production data taking. Both distributions were fitted with Gaussian functions. The centroids of these fits represent the beam center. The chicane magnetic field was optimized in such a way that the beam centroids of both lepton beams lie around the 8th fiber in both $\mathrm{x}$ and $\mathrm{y}$ orientations for both beams. The chicane magnetic field was optimized during commissioning of the experiment as described in the following section. 

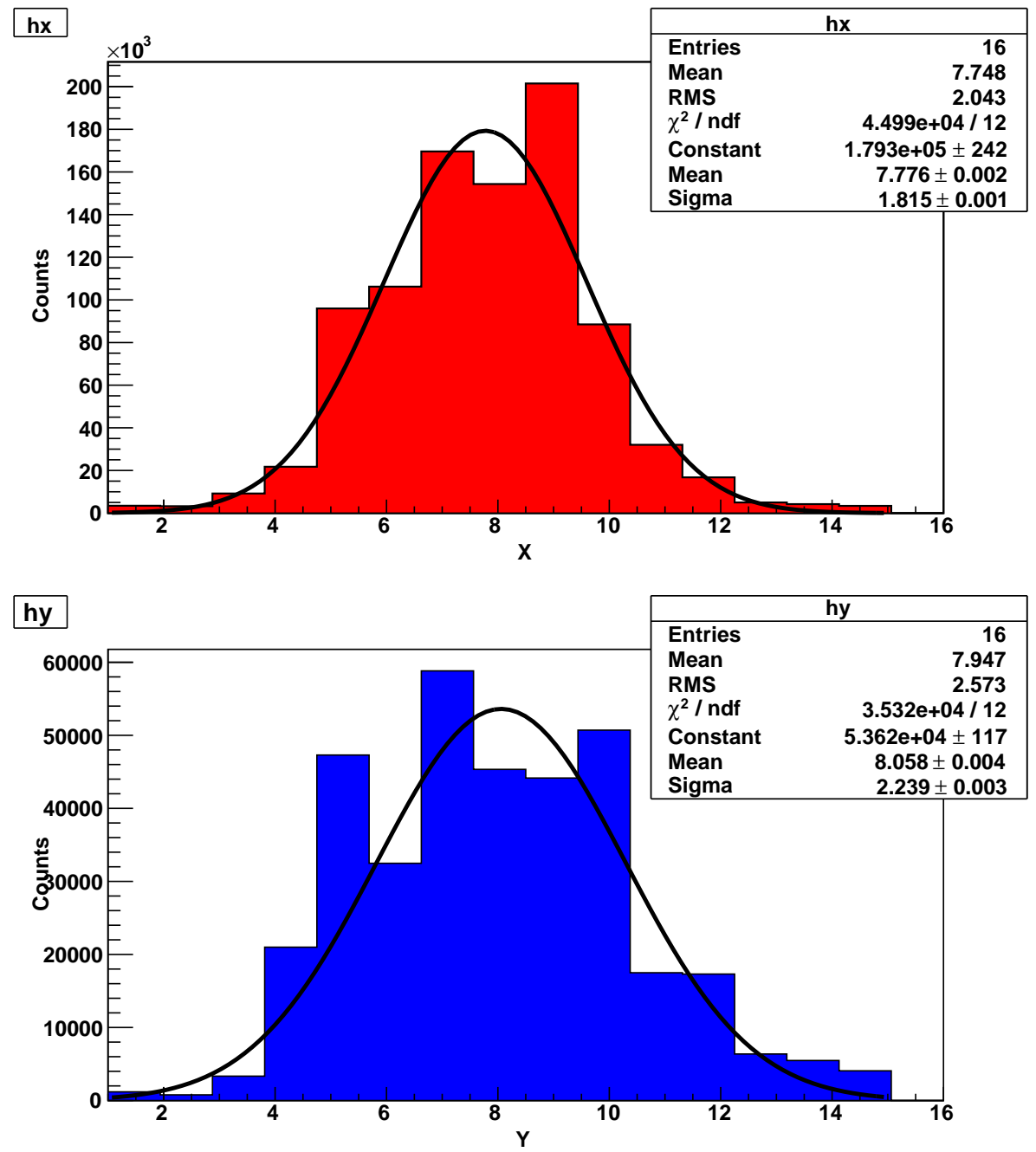

Figure 3.11: Beam position distribution along horizontal fibers (top) and along vertical fibers (bottom). The solid line is a Gaussian fit to the respective distributions.

\section{Chicane Magnetic Field optimization}

In order to find the optimum magnetic field to operate the chicane, the centroid position of each lepton beam was measured at various Italian dipole currents, while keeping the PS dipole current fixed. The other lepton beam was blocked by the lepton blocker. The beam centroid position moved to the left or to the right depending upon the ID current and lepton type. Fig. 3.12 shows the results. The ID current when the centroid positions of the left (electron) and the right (positron) beam intersect gives 
the optimum ID current when the beam centroid positions of both beams are the same. The optimum ID current from Fig. 3.12 was found to be $327.55 \mathrm{~A}$ and was set at that value throughout the experiment. Since any fluctuation in the chicane magnetic field can cause beam offsets, the position of the mixed beam was continuously monitored for stability throughout data taking.

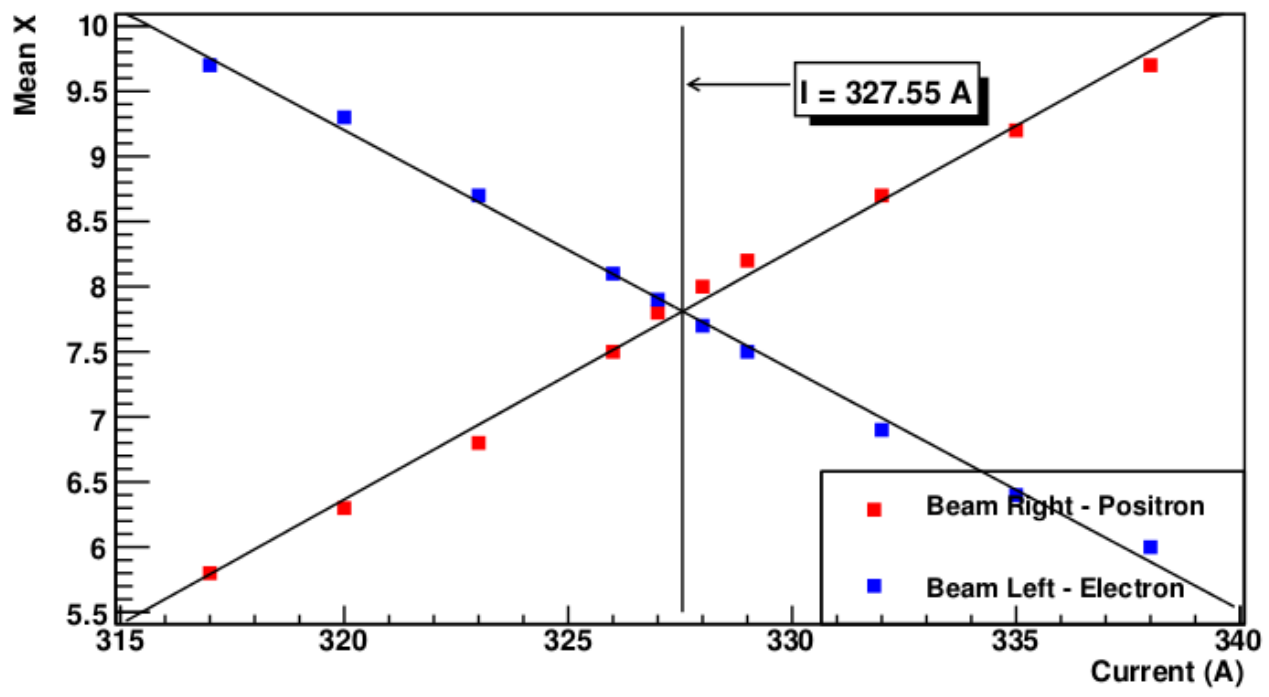

Figure 3.12: The position of the beam centroid along $\mathrm{x}$ as the function of chicane current. The blue squares are for the left beam (electron), red squares are for the right beam (positron) when the other beam is blocked. The straight line is a fit to the data points. The ID current setting where two straight lines intersects gives the optimum chicane current.

\subsubsection{Target}

The mixed beam interacted with the target located approximately 4-m downstream of the SFM. The target used in this experiment was a cylindrical 30-cm-long, 6-cm internal diameter, and 127-micron-thick Kapton cell filled with liquid hydrogen $\left(\mathrm{LH}_{2}\right)$. The center of the target was located at $30 \mathrm{~cm}$ upstream of the CLAS center. The target was cooled to a temperature of $20^{\circ} \mathrm{K}$ by circulating liquid helium in the heat 


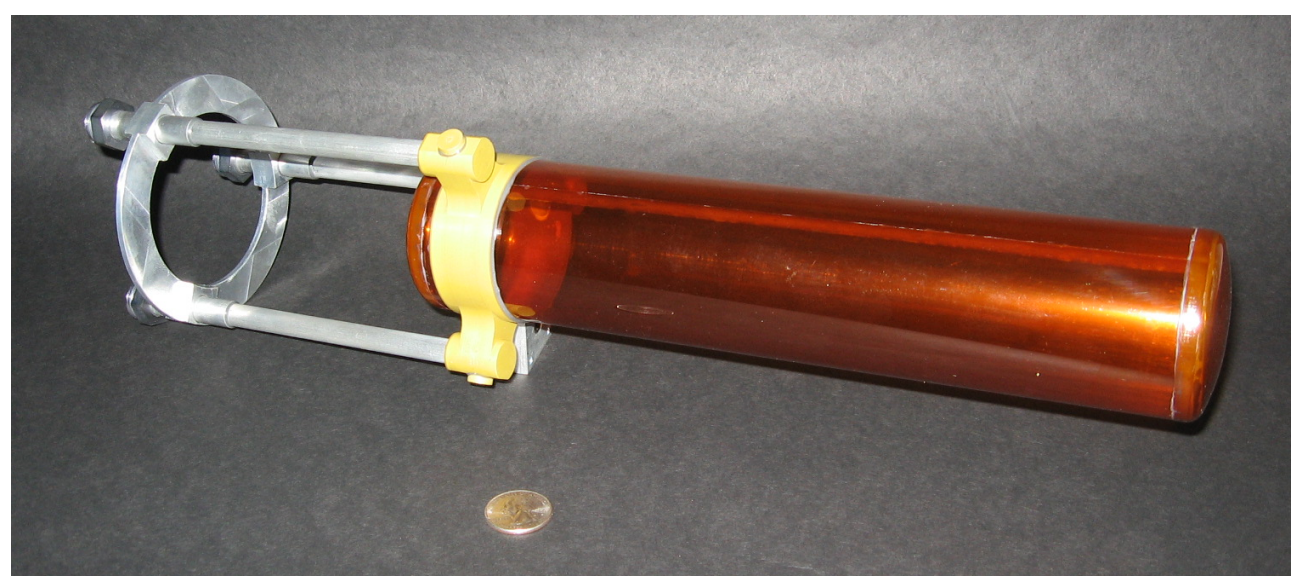

Figure 3.13: Photograph of the actual TPE target cell

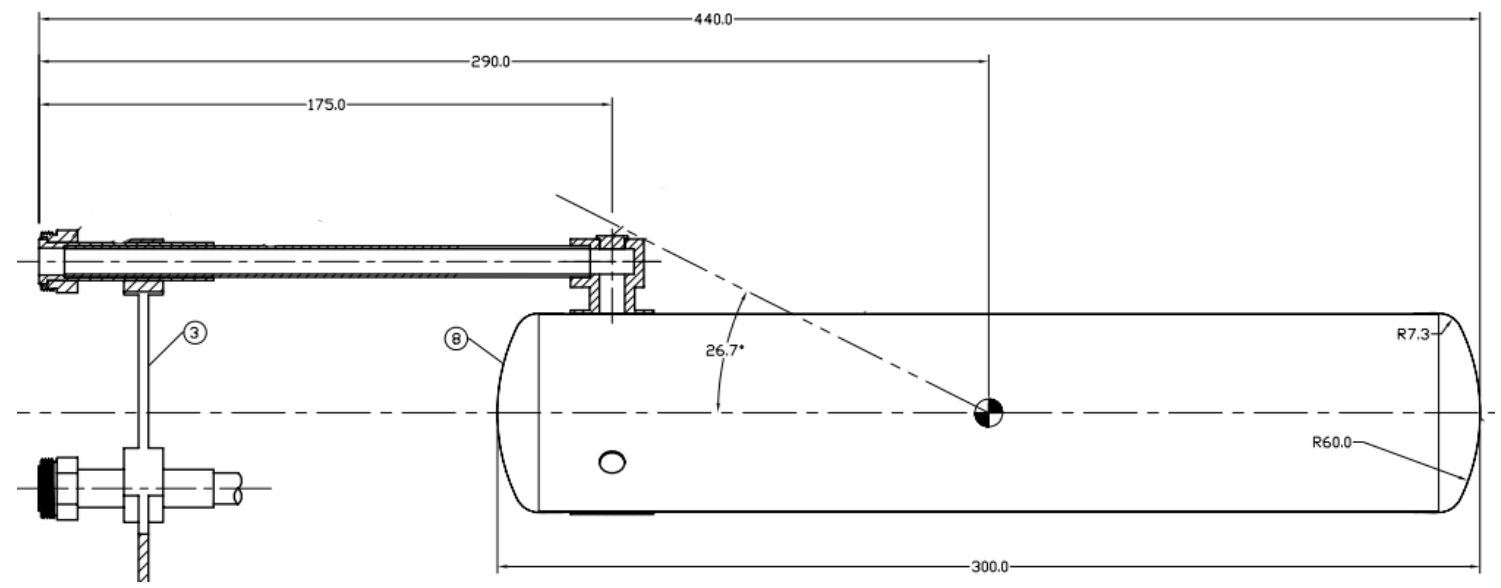

Figure 3.14: Engineering assembly drawing of the TPE Target. The dimensions are given in $\mathrm{mm}$.

exchanger attached to the cell. The target was thermally insulated with five layers of super-insulation each with 3-PLY CEREX and aluminized Mylar. The average pressure inside the cell was maintained at approximately 1100 mbar and the target density was $0.0708 \mathrm{~g} / \mathrm{cm}^{3}$. Fig. 3.13 shows a photograph of the actual target cell used in this experiment and the Fig. 3.14 shows the engineering assembly drawing of the target used in this experiment. The target was enclosed in a 75-micron thick aluminum scattering chamber. 


\subsubsection{Downstream Beam Profile System}

A downstream beam profile system was used to monitor the profiles of the lepton beams for their stability before and after each chicane field reversal. The system consisted of a Dense Fiber Monitor (DFM) and a TPE electromagnetic calorimeter (TPECal). The DFM consisted of a packed array of $2 \mathrm{~mm} \times 2 \mathrm{~mm}$ scintillating fibers in a grid mesh structure with 64 fibers in each plane. The TPECal was an electromagnetic shower calorimeter that consisted of 30 identical shashlik modules. The DFM was mounted at the front face of the TPE calorimeter (TPECal) and the

combined system was capable of moving left and right of the beam with a stepping motor. The DFM-TPECal system was moved into the beam only during beam profile measurements before and after each chicane flip. In this section we will discuss the details of the TPECal.

\section{TPE Calorimeter}

The TPCal was used to measure the beam energy distributions of both lepton beams and to check whether the energy profiles of both beams are identical. Each TPECal module consists of alternating layers of $1 \mathrm{~mm}$ lead and $2 \mathrm{~mm}$ plastic scintillator. Each module is $45 \mathrm{~cm}$ long, with a square front face of dimension $3.82 \times 3.82 \mathrm{~cm}^{2}$. The calorimeter modules are arranged in five rows inside an aluminum box. Each row contains 6 calorimeter modules. The light from an individual scintillator layer in each module is collected by 16 wavelength-shifting fibers of $1.5-\mathrm{mm}$ diameter. The fibers are $7.7 \mathrm{~mm}$ apart and are coupled to a 25-mm diameter Hamamatsu R3998-02 PMT placed in a plastic housing. The PMTs are wrapped with sheet of mu-metal ${ }^{3}$ to protect them from static magnetic fields (Fig. 3.15).

\footnotetext{
${ }^{3}$ An alloy of nickel and iron and has a very high magnetic permeability
} 

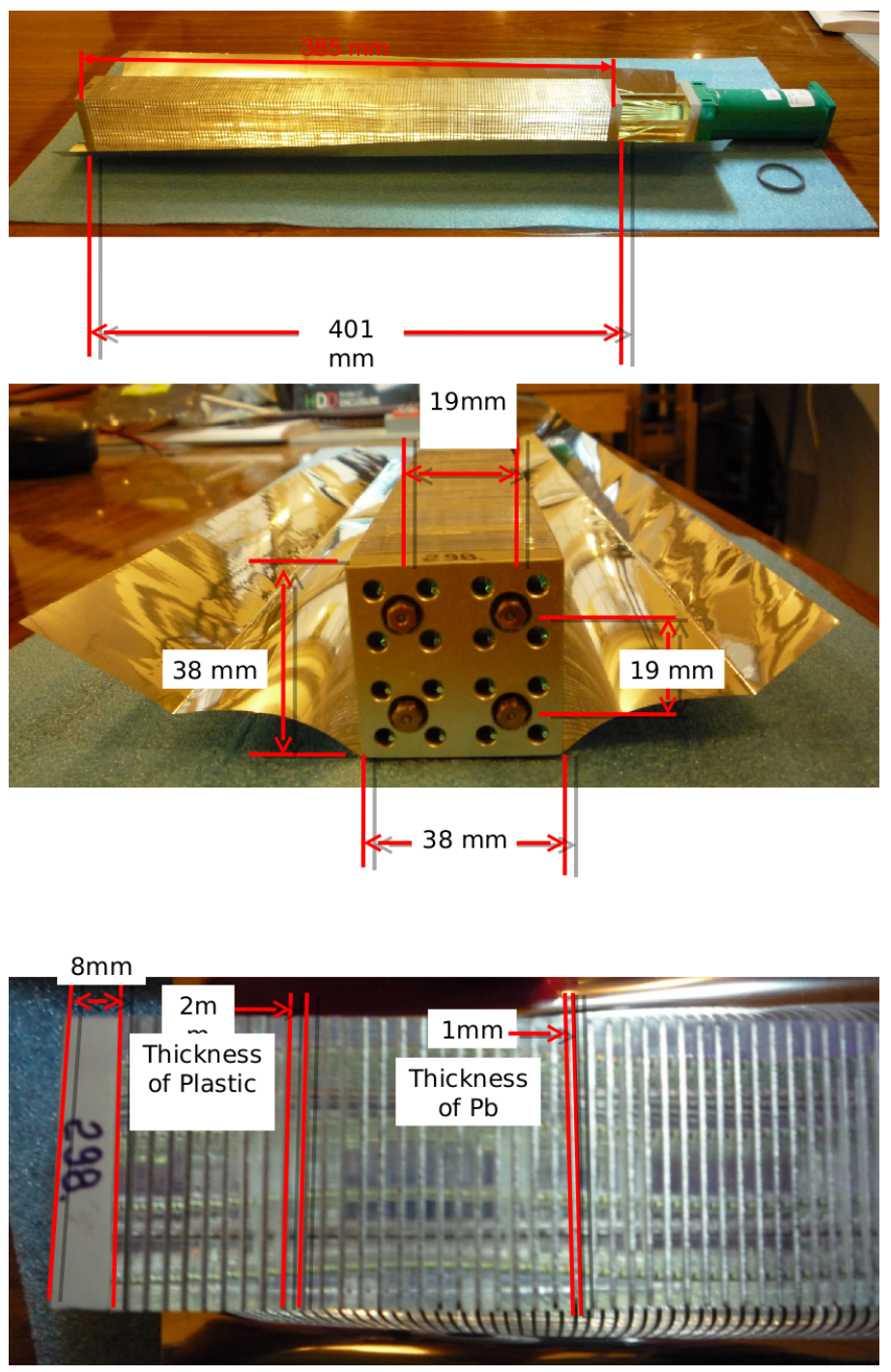

Figure 3.15: Top: Side view of a calorimeter module, showing the 45-cm length and the plastic housing for the PMT on the right side; Middle: End view of a shashlik module, showing the four bolts fastening it together and the ends of the 16 wavelength shifting fibers; Bottom: Close-up of a module showing the alternating plastic and lead layers.

The signal from each TpeCal module was split, and sent to a discriminator and, after a delay, to an analog to digital converter ADC. The signals from each discriminator were ORed to form the TpeCal trigger, the ADC gate, and the TDC common stop. The other output of each discriminator was sent to a TDC for digitization. The 


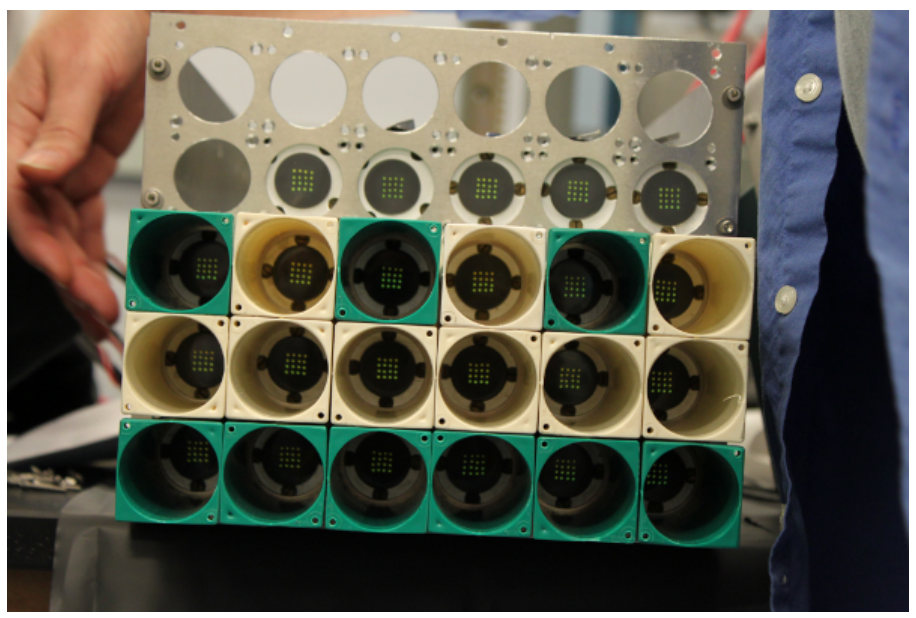

Figure 3.16: End view of the partially assembled TPE calorimeter showing 23 of the 30 shashlik modules and 18 of the PMT housings. The 16 wavelength shifting fibers (green dots) can be seen clearly.

TpeCal was mounted on the forward carriage just downstream of the CLAS EC with the ends of the shashlik modules pointed towards the target (upstream) and the PMTs pointed away from the target (downstream). A helium-bag beamline was placed between target vacuum and DFM.

During normal production data taking, the TpeCal/DFM system was kept out of the beam. However, to measure the individual beam profile, the TpeCal/DFM assembly was moved into the beam line. During such measurements, the target was emptied to reduce multiple scattering, one of the two beams (electron or positron) was blocked at the chicane by a lepton blocker, the beam intensity was reduced by a factor of about $10^{4}$ to allow counting of incident leptons [60].

\subsection{Background Control}

Since a tertiary electron-positron beam was produced from the high-current primary electron beam, excessive background was a great concern ever since the experiment was first proposed in May, 2004. Several short test runs, as well as a full scale test run, coupled with extensive computer simulations were performed to identify the major 


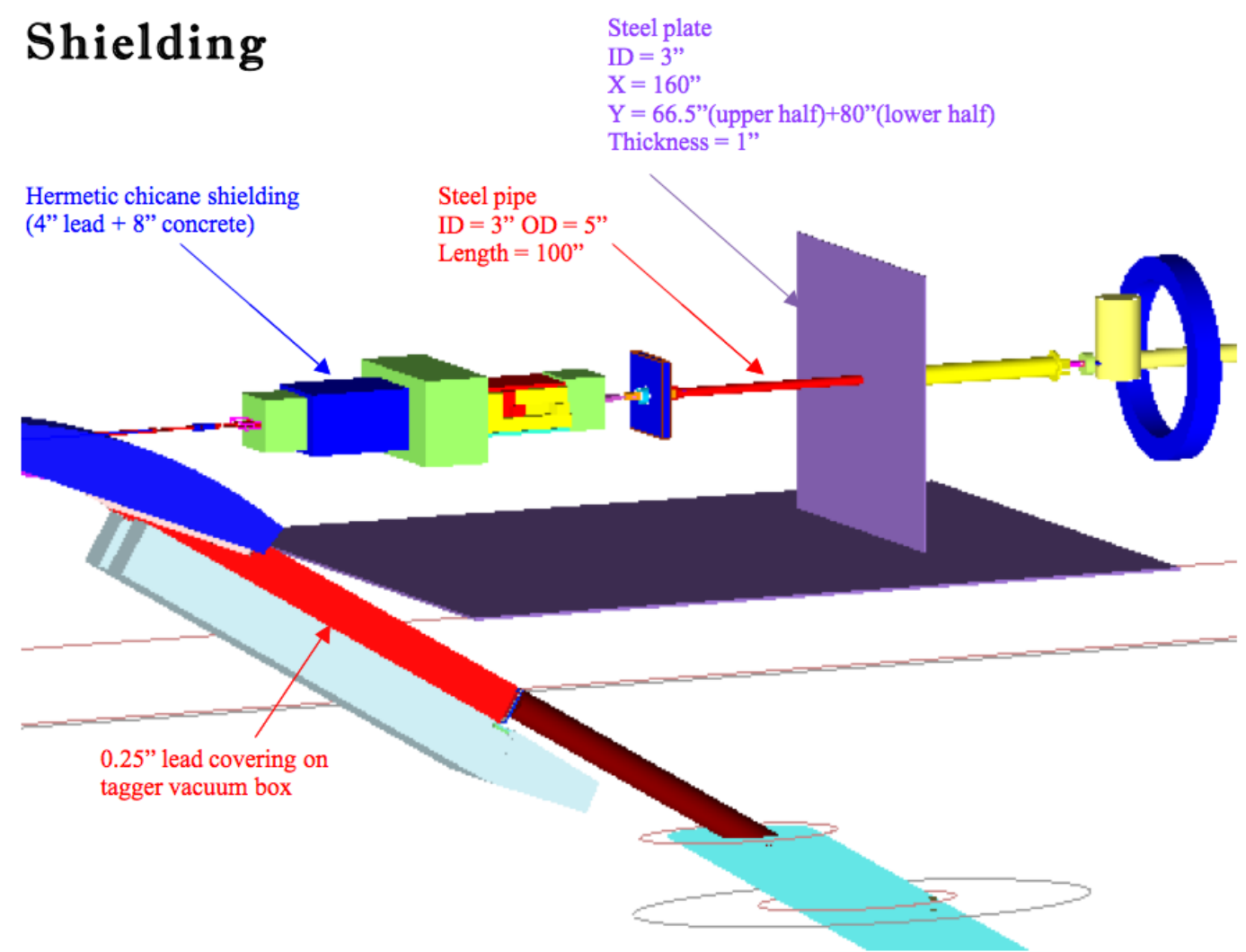

Figure 3.17: Final design of the nominal shielding components along the TPE beamline

sources of background and eliminate them [59, 61]. Based on the knowledge gathered from these engineering test runs, several shielding configurations were designed and simulated in order to find the best shielding configuration for background reduction. The designed nominal shielding configuration along the TPE beamline is shown in Fig. 3.17.

Prior to running the TPE experiment, all the components of the simulated shielding configuration were installed along the beamline. Installation of all the shielding components was an extensive task but was completed with effort from the Hall B engineering staff, collaborators from ODU and FIU. The major shielding components are: 
1. The tagger vacuum box was covered with $0.25^{\prime \prime}$ of lead sheets.

2. The tagger beam dump area, which was identified as one of the major sources of background, was covered with concrete and lead blocks.

3. The helium bag beamline, connecting each end of the PS magnet to the ID mangets, was hermetically shielded. The shielding consisted of $4^{\prime \prime}$ of lead, $8^{\prime \prime}$ of concrete, and a few inches of borated polyethylene.

4. A $4.5^{\prime \prime}$-thick lead wall with steel cladding was placed just downstream of the second ID magnet.

5. A tungsten collimator with $1.75^{\prime \prime}$ internal diameter was used at the aperture of the lead wall.

6. A $1^{\prime \prime}$-thick, $152^{\prime \prime}$ high carbon-steel shield-wall was placed downstream of the lead-wall.

7. A plastic shield surrounding the target chamber, known as a Møller shield, was used in order to reduce low-energy Møller/Bhabha leptons.

Fig. 3.18 shows photographs of some of the shielded areas along the beamline.

\subsection{Trigger and Data Acquisition System}

In CLAS, the events of interest are acquired by implementing a two level hierarchal trigger system. If the trigger condition is met, the data acquisition system takes an electronic snapshot of all the information from the detectors, constituting an "event." The CLAS level 1 trigger orders the data acquisition to process all the PMT prompt signals within $90 \mathrm{~ns}$ through a memory look up [55]. These signals include information

about the location of the hit in the TOF scintillator, and the energy deposited in the EC, etc. The level 1 trigger uses information from the PMTs to determine whether a 

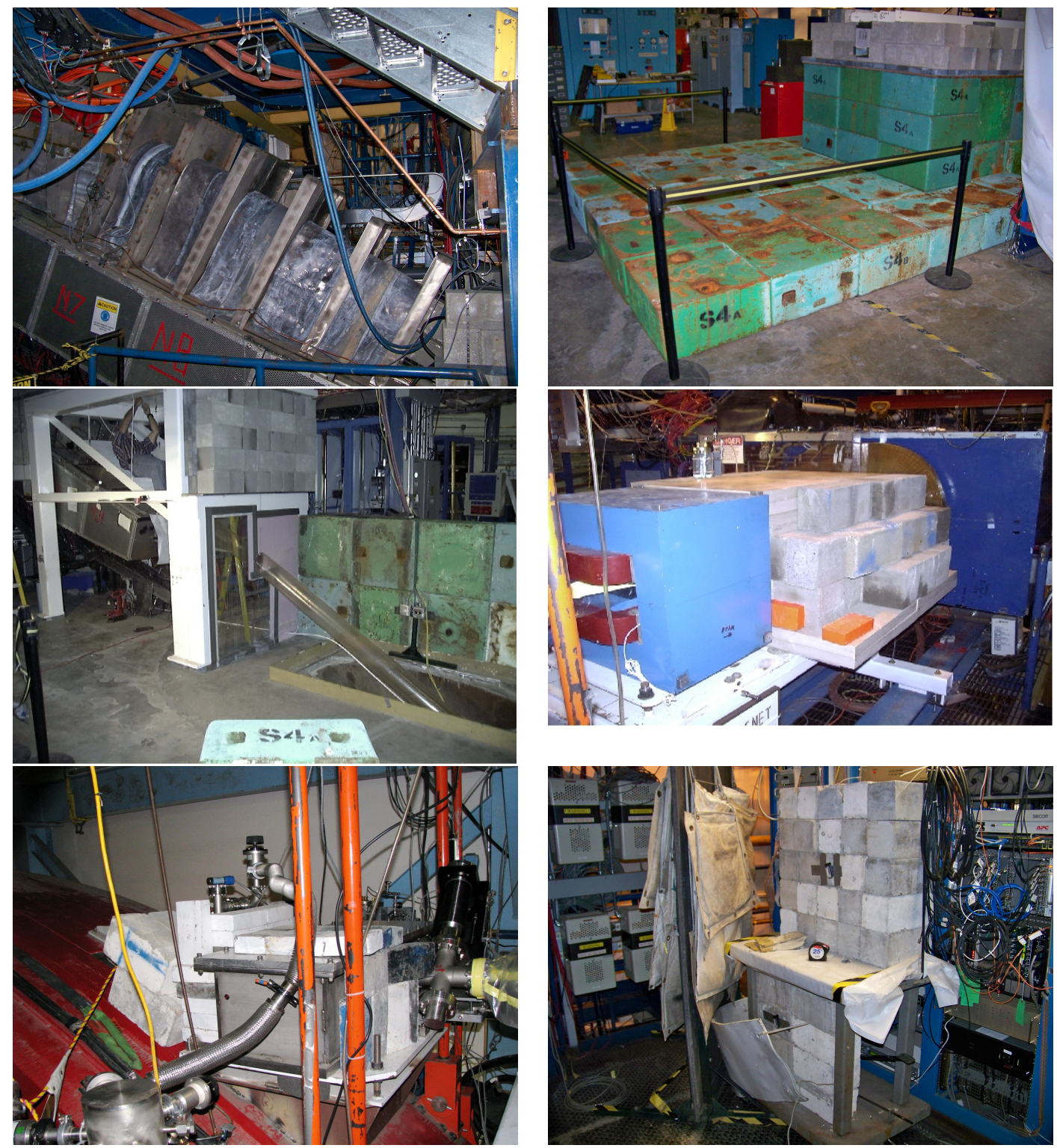

Figure 3.18: Photographs taken after the completion of beamline shielding work. Top left: tagger vacuum box shielding with lead sheet. Top right: Tagger bunker shield. Middle left: Shielded tagger vacuum box and beam dump area. Middle right: The region between the first Italian dipole and the PS dipole. Bottom left: photon collimator shielding area. Bottom right: protective shielding for electronic devices. 
desired event has occurred. For fast response, the bit patterns from these detectors are compared to the preloaded patterns in the memory tables [55]. The level 1 trigger condition is occasionally set by undesired accidental events, which lack matching drift chamber trajectories. In order to reject these events, level 2 triggers are employed. The primary trigger for the TPE experiment required a hit in the TOF panel $1(\theta<$ $45^{\circ}$ ) and the EC in the same CLAS sector, in coincidence with a TOF hit anywhere in the opposite sector. This experiment did not implement a level 2 trigger. The data from the various detector components of CLAS were digitized in 24 FASTBUS and VME crates and collected by VME Readout Controllers (ROCs). The arrays of the digitized outputs were combined to build an event by using the simple event builder (SEB) system. Once a complete event is built, the Event Recorder was used to write the event information directly to the RAID disks [55]. Then, the data from these disks were transfered to the computer center, where they were stored and accessed for physics analysis. The events were recorded in the units called "runs" with approximately 20 million trigger events in each run.

\subsection{Data Collection}

During the alloted run period, the TPE experiment collected nearly 1000 runs, which had approximately 12 billion trigger events in the standard experimental condition. Table 3.1 summarizes those experimental conditions.

The data were collected with both positive and negative torus magnet polarities in order to minimize systematic uncertainties arising from lepton acceptance differences. The lepton acceptance difference can arise from the detector imperfections such as holes in the drift chamber, bad TOF, bad EC regions, etc., that are seen differently for inbending and outbending leptons. Data were also collected for both positive and negative chicane magnet polarities in order to account for any left-right asymmetry in the electron/positron beam. The magnet polarities were periodically reversed, and 
Table 3.1: TPE standard experimental conditions

\begin{tabular}{cc}
\hline \hline Component & Value \\
\hline Primary Beam Current & $100<\mathrm{I}<120 \mathrm{nA}$ \\
Primary Beam Energy & $5.6 \mathrm{GeV}$ \\
Radiator & $0.009 \mathrm{RL}$ \\
Converter & $0.09 \mathrm{RL}$ \\
Pair Spectromer (PS) & $\pm 676.5 \mathrm{~A}$ \\
Italian Dipole (ID) & $\pm 327.7 \mathrm{~A}$ \\
Tagger Current & $1927 \mathrm{~A}$ \\
Torus Current & $\pm 1500 \mathrm{~A}$ \\
Mini-Torus Current & $4000 \mathrm{~A}$ \\
\hline \hline
\end{tabular}

a roughly equal number of events were collected in each magnet cycle. A complete magnet cycle includes runs from all the possible combinations of torus and chicane magnet polarity.

The data were collected for four complete cycles of the chicane and the torus magnet polarity reversals. Table 3.2 shows the summary of the collected data and the magnet polarities reversals during the course of the experiment. TPE calorimeter runs were taken before and after each chicane polarity reversals to check stability of the lepton flux/energy.

Table 3.2: Summary of the collected data

\begin{tabular}{|c|c|c|c|c|c|}
\hline \hline Magnet Cycle & Date & Torus Polarity & PS Polarity & Runs & Total Events (in millions) \\
\hline \multirow{5}{*}{1} & $12 / 08 / 2010$ & - & - & $66228-66322$ & 494 \\
& $12 / 13 / 2010$ & - & + & $66323-66377$ & 507 \\
& $12 / 16 / 2010$ & + & + & $66381-66490$ & 949 \\
& $12 / 20 / 2010$ & + & - & $66517-66540$ & 265 \\
\hline \hline \multirow{5}{*}{2} & $01 / 06 / 2011$ & + & - & $66567-66646$ & 794 \\
& $01 / 11 / 2011$ & - & - & $66651-66700$ & 688 \\
& $01 / 14 / 2011$ & - & + & $66718-66773$ & 741 \\
& $01 / 18 / 2011$ & + & + & $66778-66850$ & 723 \\
\hline \hline \multirow{3}{*}{3} & $01 / 23 / 2011$ & + & - & $66873-66939$ & 764 \\
& $01 / 29 / 2011$ & - & - & $66944-66995$ & 739 \\
& $02 / 02 / 2011$ & - & + & $66999-67041$ & 768 \\
& $02 / 04 / 2011$ & + & + & $67043-67092$ & 748 \\
\hline \hline & $02 / 08 / 2011$ & + & - & $67106-67152$ & 793 \\
& $02 / 11 / 2011$ & - & - & $67155-67218$ & 954 \\
& $02 / 15 / 2011$ & - & + & $67252-67298$ & 392 \\
& $02 / 18 / 2011$ & + & + & $67290-67329$ & 821 \\
& $02 / 22 / 2011$ & - & + & $67343-67383$ & 198 \\
\hline \hline
\end{tabular}




\subsection{Event Reconstruction}

The data on the disk contains information about the recorded events in the form of digital output signals from TDCs and ADCs. The raw data were reconstructed into meaningful physical quantities by a process known as "data cooking." The data were cooked using the CLAS REConstruction and AnalySIS (RECSIS) software package. The event reconstruction process starts with the drift chamber hit-based tracking. In hit-based tracking, information about the position of wires with a hit in a given sector is gathered. The adjacent hits in each drift chamber super-layer are then grouped into hit clusters. The hit clusters are then linked across the three regions of the DCs to produce a viable hit-based track. The sign and magnitude of the curvature of this track upon traversing through the $\mathrm{R} 2$ drift chamber gives the charge, and the magnitude of the momentum of the track [56].

In the second step, known as time-based tracking, the hit-based tracks are extrapolated to find a viable hit in the corresponding TOF panel. If a hit is found, the timing measurement is used to set an upper limit on the times of the drift chamber hits associated with that track. The hit clusters without viable timing information are removed. The track is then re-fit using only the remaining clusters. This process is repeated a few times to refine the momentum measurements as well as the measurement of the event vertex, which is determined by the distance of closest approach of the track to the beamline. After the track parameters are well defined in the timebased tracking, the track is extrapolated to the rest of the CLAS components. If the corresponding hits are recorded in those components, the information is added to the track description in the corresponding data bank [56]. The software then writes the output information in a BOS file format, which is then converted into ROOT format for physics analysis.

\footnotetext{
${ }^{4}$ An Object-Oriented data analysis framework 62
} 


\subsection{Detector Calibrations}

Each detector component of CLAS is generally calibrated for each experimental run period. The purpose of the calibration is to optimize the momentum and energy information using the collected data. The different components of CLAS were calibrated prior to "cooking" the data. This section will discuss the details of the DC calibration performed by this author.

\subsubsection{Drift Chamber Calibrations}

When a charged particle traverses through CLAS drift chambers, on average 30 out of the 34 layers of the sense wires are hit. The track of the the charged particle is obtained by least square fitting the positions of these hits [56]. The distance of this fitted track from the sense wire is known as the Fitted Distance of Closest Approach (FITDOCA). Additionally, the distance of the track from the sense wire can also be calculated from the drift time of the particle using a drift distance function and is known as the Calculated Distance of Closest Approach (CALCDOCA). The drift distance function gives a relation between the drift distance and the drift time $(t)$ [56]. For a given track entrance angle, the drift distance function follows a form given by Eq. 3.1 in the R3 drift chambers:

$$
x(t)=v_{0} t+\eta\left(\frac{t}{t_{\max }}\right)^{p}+\kappa\left(\frac{t}{t_{\max }}\right)^{q},
$$

where $v_{0}$ is the drift velocity at $t=0, t_{\text {max }}$ is the maximum drift time for every sector and every superlayer, and the parameters $\eta, \kappa, p$, and $q$ are determined by fitting the time to distance correlation in each superlayer in each sector [56]. Similarly, the drift distance function follows a polynomial form given by Eq. 3.2 in the R1 and R2 drift 
chambers [56].

$$
x(\hat{t})=a \cdot \hat{t}+b \cdot \hat{t}^{2}+c \cdot \hat{t}^{3}+d \cdot \hat{t}^{4}+(c c-a-b-c-d) \cdot \hat{t}^{5},
$$

where $a, b, c, d$, and $c c$ are determined by a fit to the drift time to drift distance correlation. $\hat{t}$ is the normalized time given by $\hat{t}=\frac{t}{t_{\max }}$.

The difference between FITDOCA and CALCDOCA is called the time residual (RESI), which measures the resolution of the drift chamber.

$$
\text { RESI }=\text { FITDOCA }- \text { CALCDOCA. }
$$

The goal of the drift chamber calibration was to minimize this residual by finding an optimum set of parameters in the drift distance function for each superlayer in each sector. The drift chamber calibration was an iterative process and started with copying over parameters from the previous CLAS experiment as initial parameters for the drift distance function. A portion of the data were cooked with those parameters. Then, a new set of parameters was obtained by re-fitting the drift distance function with the newly cooked data. The process was repeated until the fit parameters converged. The parameters were finally adjusted by fitting the residual distributions for each super layer in each sector with a double-Gaussian function. The centroid and the width of this fit represents the DC mean and sigma. Fig. 3.19 shows the distribution of the residual in sector 1 superlayer 1 (S1SL1). The quality of the DC calibration was monitored by plotting the position of DC mean and sigma of this fit against the run number. Fig. 3.20 and Fig. 3.21 show the stability of the DC mean and sigma over the TPE run period. The vertical lines on the plots show the magnet polarity reversals. As has been discussed earlier, this experiment had to deal with significant number of background related hits in the DC. As a results, the chambers 


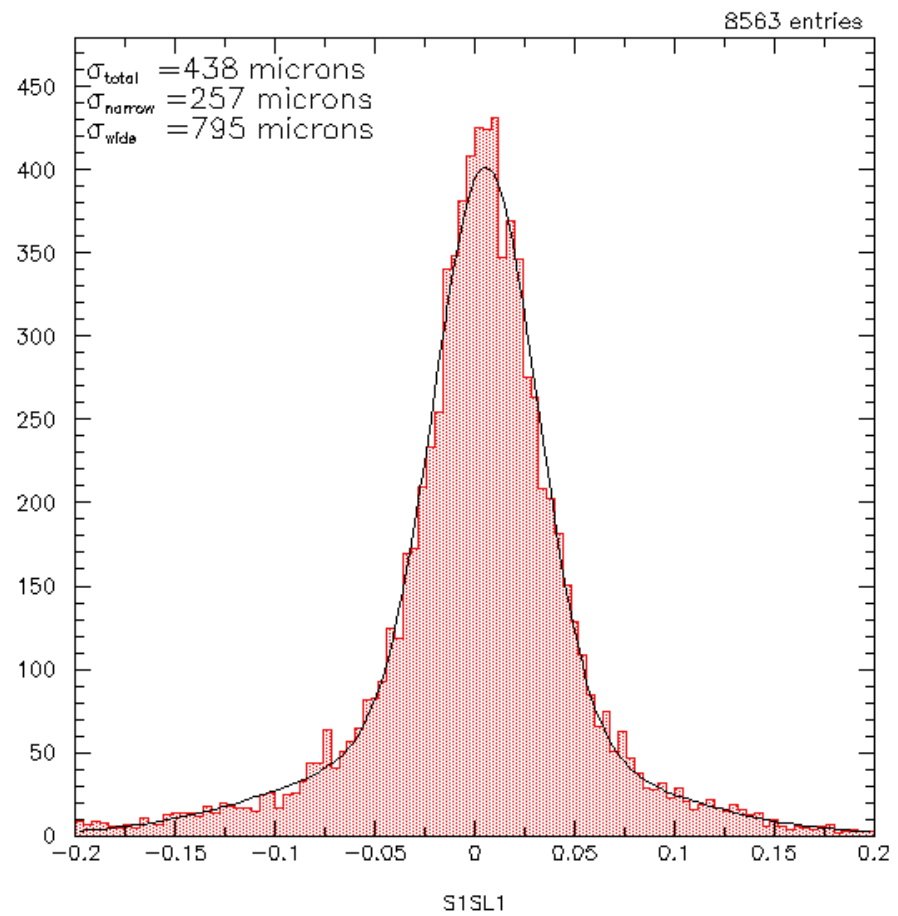

Figure 3.19: Distribution of the residual in S1SL1. The black curve is the doubleGaussian fit to the residual distribution.

were significantly nosier than usual CLAS experiments. However, the fluctuation of the DC mean and sigma were still within the CLAS tolerance [56].

\subsection{Kinematic Corrections}

A charge particle traversing through CLAS loses energy along its track via interaction with the detector elements. In order to account for the energy loss, the reconstructed energies are usually corrected. In order to account for inadequate knowledge of the magnetic field and for drift chamber dislocations, the reconstructed momentum of the charged particle are also corrected. This section will discuss the energy loss and the momentum corrections performed for this experiment. 


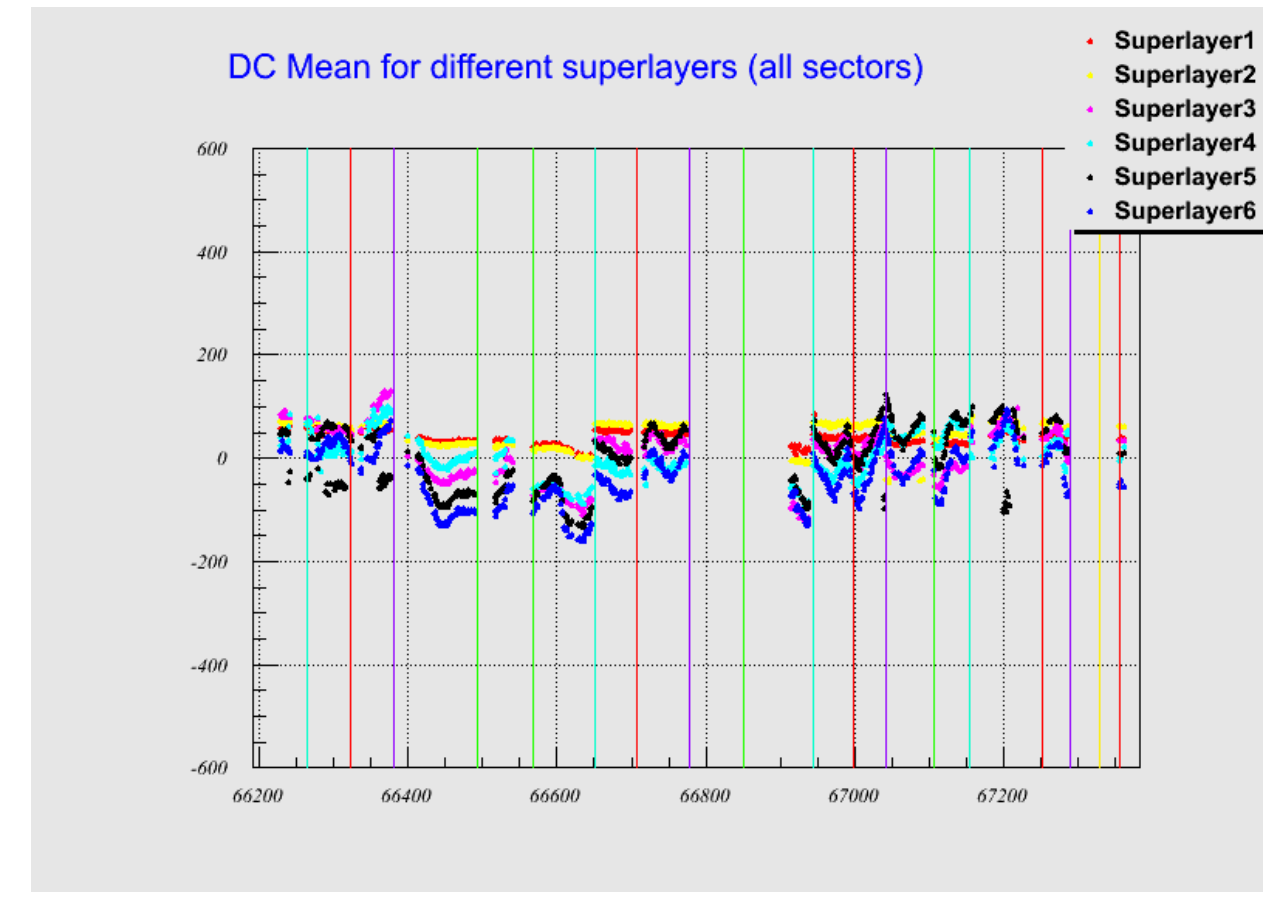

Figure 3.20: DC mean vs. run number

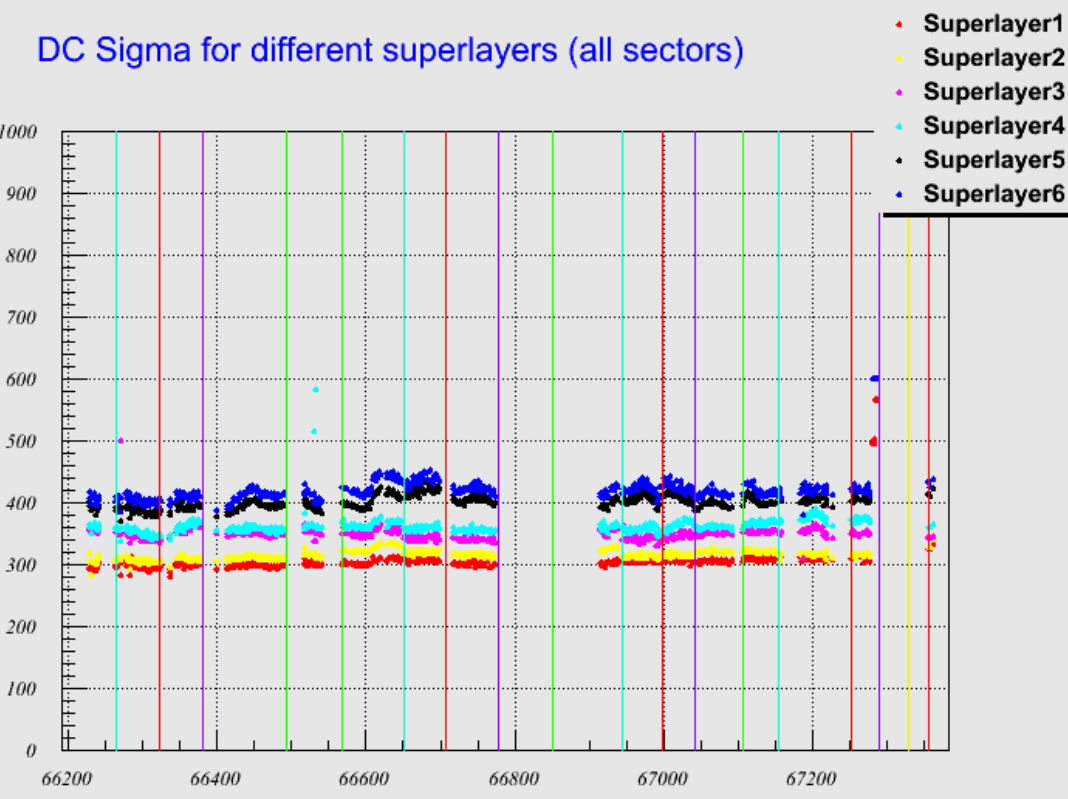

Figure 3.21: DC sigma vs. run number 


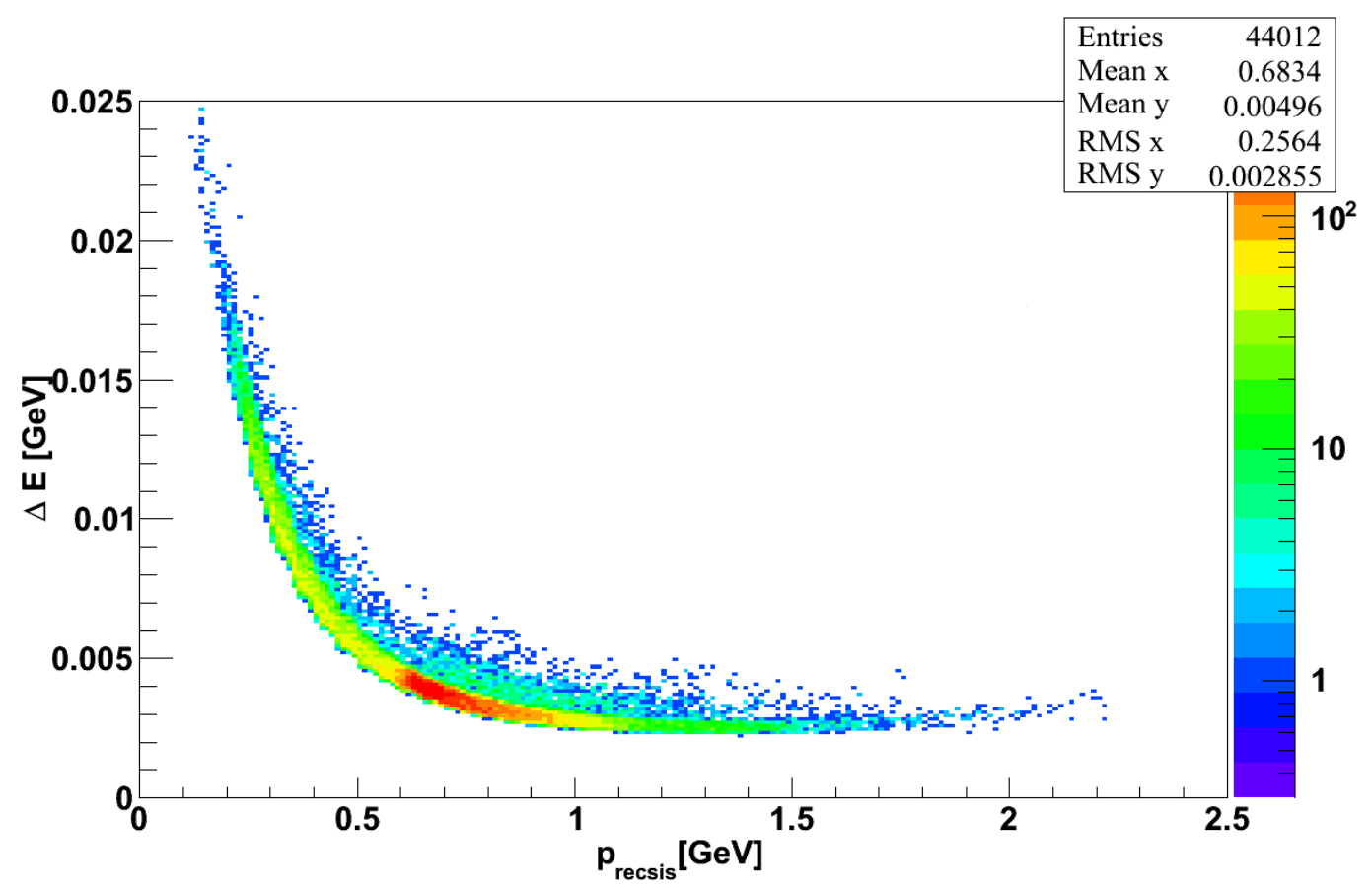

Figure 3.22: Energy loss correction for the proton tracks

\subsubsection{Energy Loss Corrections}

The CLAS reconstruction software gives an effective momentum between Region 1 and Region 3 drift chambers, without accounting for the energy loss by the charged particle along its track. The energy loss is significant for the low momentum protons. In order to correct for this loss, the standard CLAS ELOSS package [63] was used. ELOSS corrects for the energy loss of the charged particle as it traverses from the event vertex through the target volume, the target cell walls, insulation, chamber windows etc. The ELOSS package uses the Bethe-Bloch equation to relate the material characteristics and path length to the energy loss [18]. Fig. 3.22 shows the energy loss correction $(\Delta E)$ versus the momentum reconstructed by RECSIS ( $\left.p_{\text {recsis }}\right)$ for protons. The low momentum protons have higher corrections while the corrections are smaller for higher momentum protons. 


\subsubsection{Momentum Corrections}

This section describes the method employed to correct the measured lepton and proton momenta reconstructed by RECSIS. This method closely follows the momentum corrections method employed by the CLAS e6 run group [64. Because of inadequate knowledge of the magnetic field and drift chamber positions, the momenta reconstructed in CLAS show some systematic deviations. This is evidenced by shifted and broadened peaks in missing mass and invariant mass distributions. For example (see Fig. 3.23) the centroid of the $W$ distribution of the $p\left(e, e^{\prime}\right)$ elastic peak is shifted from its known value $W=M_{p}=0.938 \mathrm{GeV}$ and is broad.

In order to correct for these deviations, the reconstructed particle momenta and angles need to be corrected. To calculate these corrections, we need a precise calculation of the invariant mass $W$, which requires precise knowledge of the incident lepton energy. As already discussed, The TPE production data were collected with a tertiary $e^{+} / e^{-}$ beam with a continuous distribution of energies from 0.5 to $5 \mathrm{GeV}$. Hence for the calibration purpose, we took special runs with a primary electron beam energy of $2258 \mathrm{MeV}$. We determined the momentum corrections for this set of data, and then applied these corrections directly to the TPE production data.

In order to calculate the required correction we employ the kinematics of elastic electron scattering. For this, we compare the measured momentum $\left(p_{\text {meas }}\right)$ of the electron with the momentum calculated $\left(p_{\text {calc }}\right)$ by using Eq. 2.8. Fig. 3.24 shows the fractional deviation of the measured electron momentum from the calculated momentum $\left(\frac{\Delta p}{p_{\text {meas }}}\right)$ with $\Delta p=p_{\text {meas }}-p_{\text {calc }}$, as a function of the azimuthal angle. The deviation clearly depends on the azimuthal angle of the scattered electron in each CLAS sectors. We also need to correct the angle $(\theta)$ of the scattered particle. We assume that the azimuthal angles $(\phi)$ of the scattered particles are correctly 


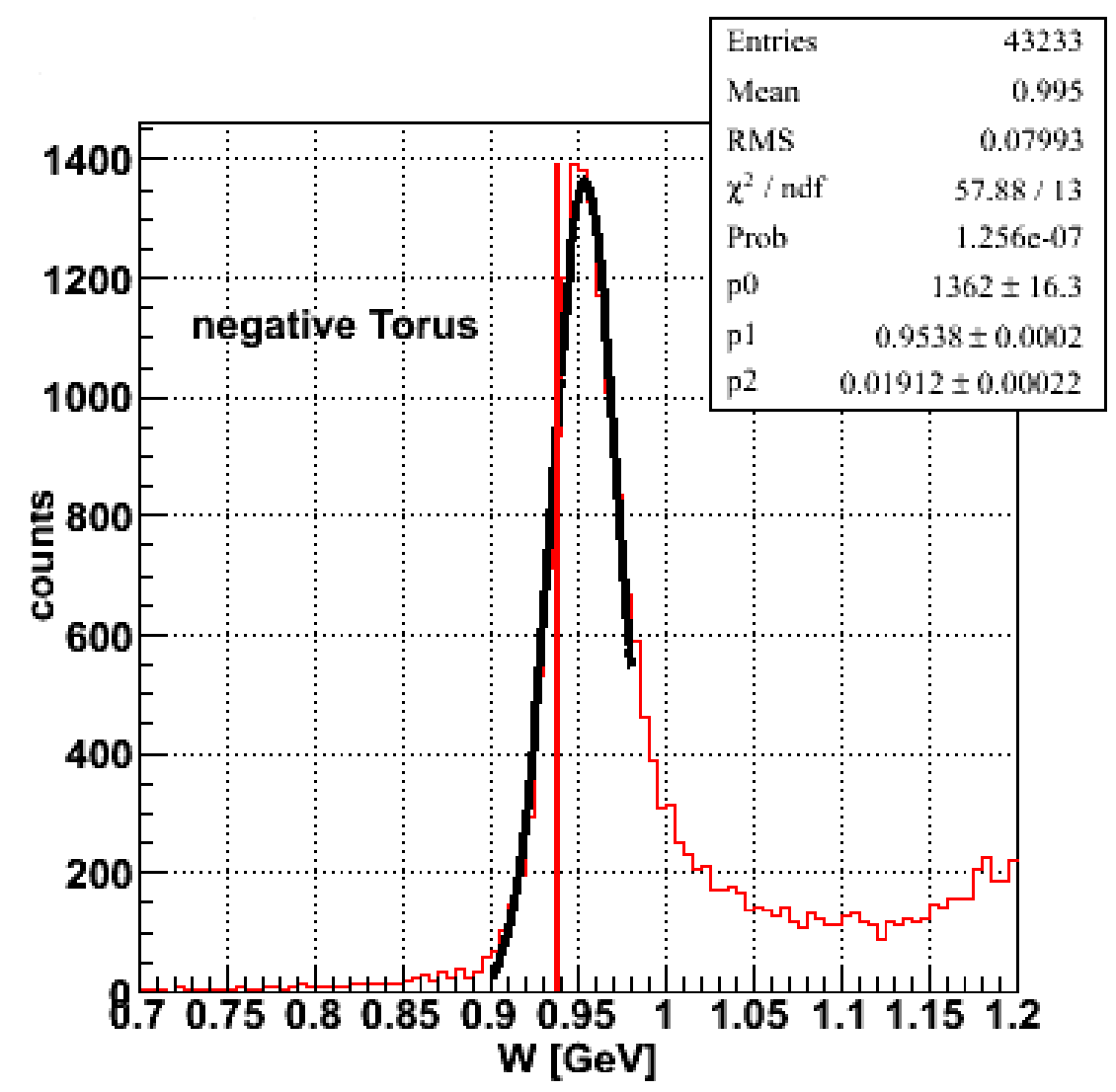

Figure 3.23: Invariant mass distribution, $W$, for all CLAS sectors before applying momentum corrections. Red curve is the actual distribution and the black curve is a Gaussian fit to the distribution. The vertical line shows the proton mass (0.938 $\mathrm{GeV})$.

determined since we found that the lepton-proton elastic events were coplanar (i.e. $\left.\phi_{l}-\phi_{p} \approx 180^{\circ}\right)$. Following Ref. [64], the correction required for the polar angle $(\Delta \theta)$ and the momentum $(\Delta p)$ are parametrized in terms of the effect of the DC dislocations on each track as,

$$
\Delta \theta=(A+B \phi) \frac{\cos \theta}{\cos \phi}+(C+D \phi) \sin \theta
$$



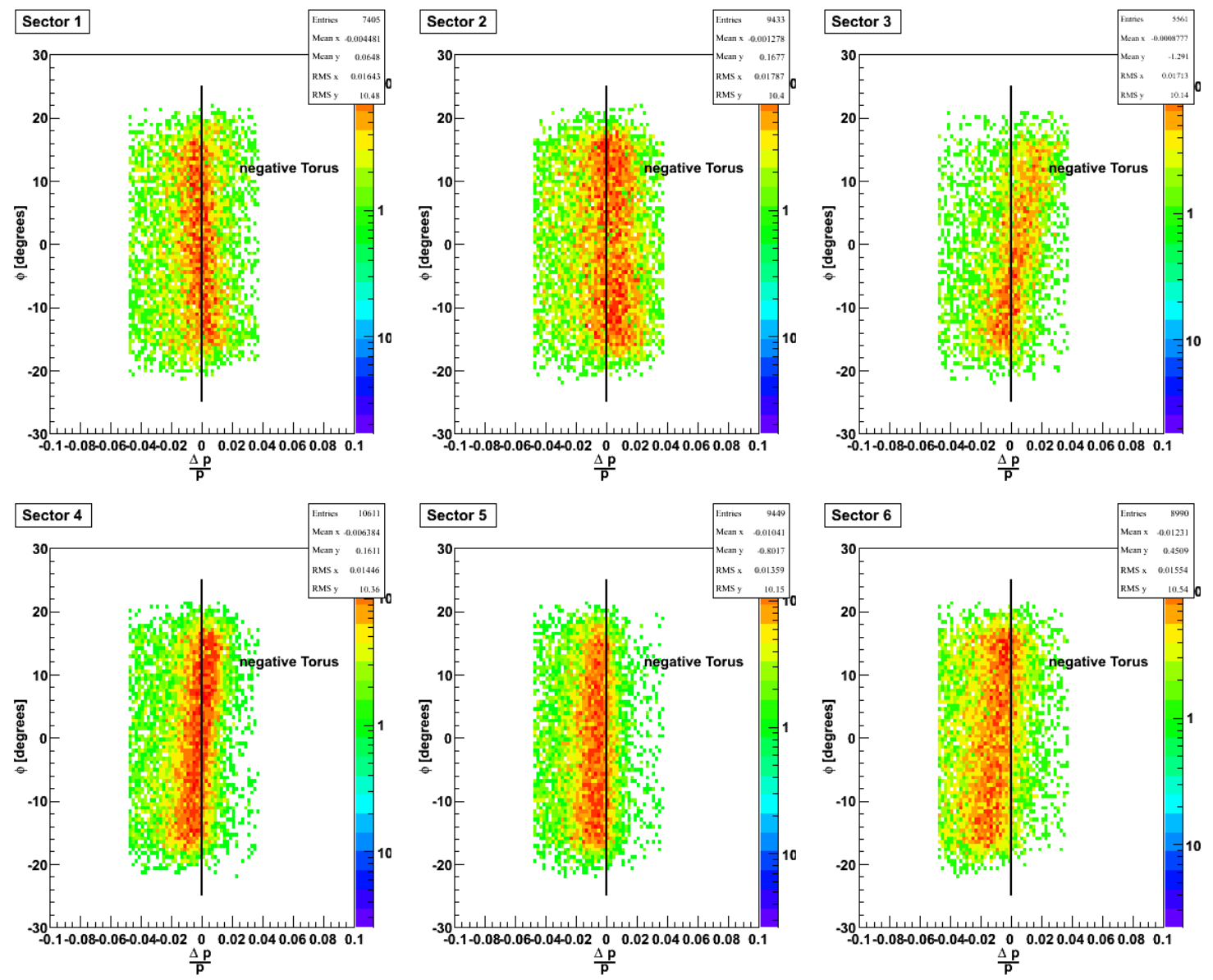

Figure 3.24: $\phi$ versus $\frac{\Delta p_{p}}{p}$ distribution in each CLAS sector before before applying momentum corrections.

and,

$$
\frac{\Delta p}{p}=\left((E+F \phi) \frac{\cos \theta}{\cos \phi}+(G+H \phi) \sin \theta\right) \frac{p}{q B_{\text {torus }}} .
$$

where:

- $q$ is the charge of the particle.

- $p$ is the reconstructed momentum in GeV.

- $\theta$ is the reconstructed polar angle. 
- $\phi \in\left[-\frac{\pi}{6}, \frac{\pi}{6}\right]$ is the reconstructed azimuthal angle relative to the mid-plane of the sector.

- The quantity $B_{\text {torus }}$ gives the integral $\int \mathbf{B}_{\text {trans }} \cdot \mathbf{d l}$ along the track path in $\mathrm{GeV}$.

The ratio $\frac{q B_{\text {torus }}}{p}$ in Eq. 3.5 is proportional to the curvature of the track and quantifies the effect of drift chamber misalignment. The simplest parameterization of this integral is given as:

$$
B_{\text {torus }}=0.76 \frac{I_{\text {torus }} \sin ^{2} 4 \theta}{I_{\max } \theta} \text { if } \theta<\frac{\pi}{8}
$$

and

$$
B_{\text {torus }}=0.76 \frac{I_{\text {torus }}}{I_{\max } \theta} \text { if } \theta>=\frac{\pi}{8}
$$

where $I_{\text {torus }}$ is the CLAS torus current and $I_{\max }=3375 \mathrm{~A}$ is the maximum allowed torus current.

In equations 3.4 and 3.5 , the parameters $A$ through $F$ account for the effects due to misalignment of the drift chambers in different directions. Parameters $A$ and $E$ describe the radially outward displacement of the chambers, which primarily affect particles in the forward direction. Parameters $B$ and $F$ describe the $\phi$-dependent radial displacements, $C$ and $G$ describe displacement along the beam axis, which primarily affects the particles at back angles, and parameters $D$ and $F$ correspond to a rotation around the radial direction.

It should be noted that the momentum correction in Eq. 3.5 depends on the particle charge, the magnitude of the CLAS magnetic field, and the track momentum itself. In addition to the angle and momentum corrections due to misaligned drift chambers, we used the parametrization given in Ref.64] to account for the effect of incorrect 
magnetic field mapping.

$$
f(\theta)=(J \cos \theta+K \sin \theta+L \sin 2 \theta)+(M \cos \theta+N \sin \theta+O \sin 2 \theta) \phi
$$

The corrections given by Eq. 3.8 are independent of the particle's charge, its momentum, and torus field strength. Hence, this correction is different from that given by Eq. 3.5 under the reversal of charge and magnetic field polarity. In order to cover a wide range of CLAS kinematics, it is important to use data samples with both in-bending and out-bending particles with a wide range of kinematics.

We determine the parameters $A-O$ by selecting exclusive events where all the particles in the final state are detected. We then employ four-momentum conservation to extract a "goodness of fit" $\left(\chi^{2}\right)$ like variable that we optimize for all the events.

\section{Event Selection for Fitting}

For fitting, we selected exclusive events, where all the particles in the final state are detected. We selected $p\left(e, e^{\prime} p\right)$ elastic events as the primary sample. To cover lower proton momenta as well as back angle leptons, we choose $p\left(e, e^{\prime} p \pi^{+} \pi^{-}\right)$exclusive events by placing cuts on missing energy and momenta. We choose runs from positive and negative torus polarity to include both in-bending and out-bending particles. For all the events the usual fiducial and target vertex cuts (discussed in Chapter 4) have been used. Once we have selected the exclusive events, all the 14 parameters for each sector are optimized by fitting in a ROOT-based fitting routine. The output of the code is an optimized set of 84 parameters, 14 for each sector. 

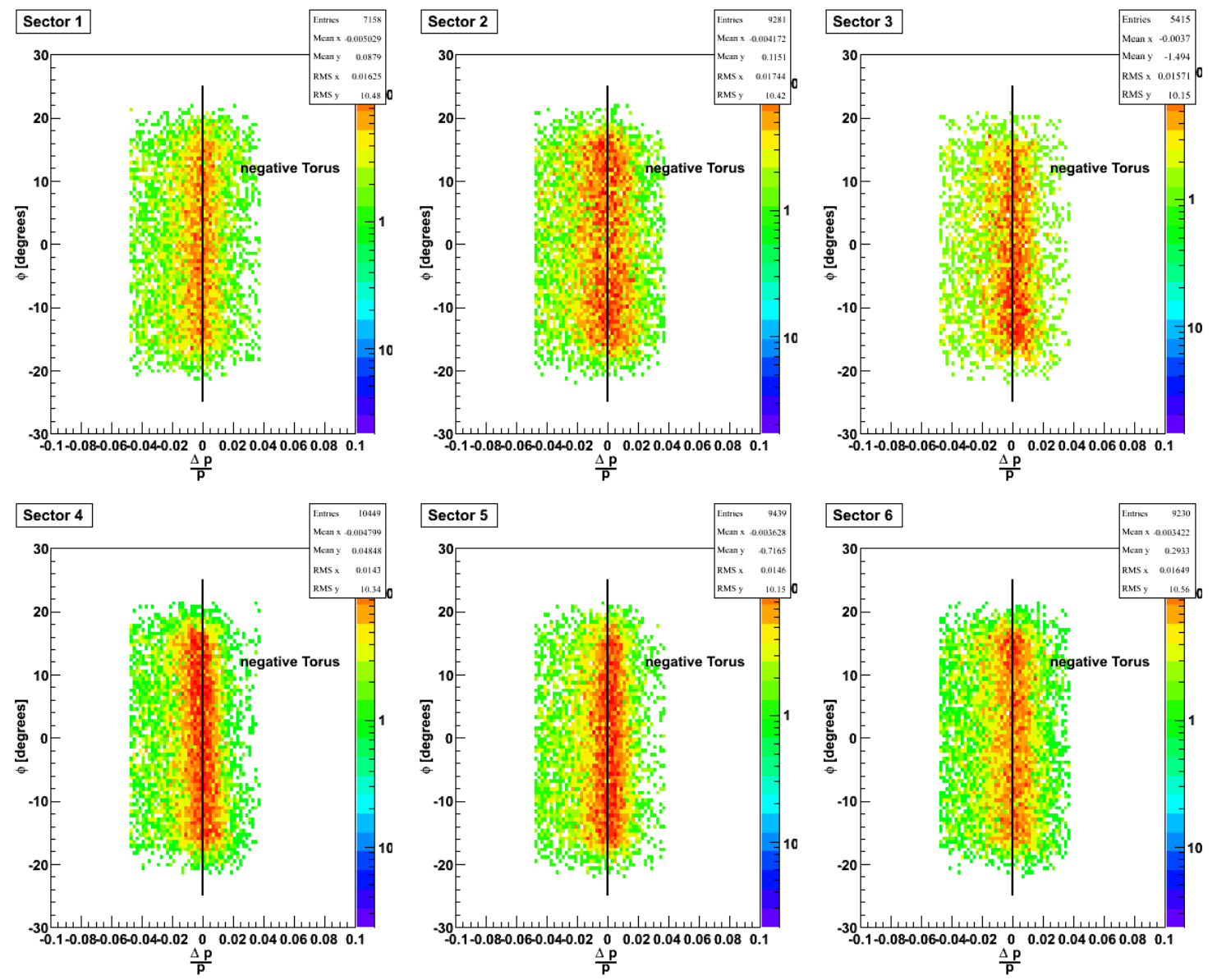

Figure 3.25: $\phi$ versus $\frac{\Delta p_{p}}{p}$ distribution in each CLAS sector after applying momentum corrections.

\section{Fitting Procedure}

We started our fitting procedure with the momentum corrections based on the CLAS e6 [64] parameters, applied energy loss corrections and calculated the missing four momentum. With the missing energy and the components of the missing threemomentum, we added squares of these components normalized to a reasonable resolution, to get an overall $\chi^{2}$ of the fit,

$$
\Delta \chi^{2}=\frac{E_{m i s s}^{2}+\left(P_{m i s s}^{z}\right)^{2}}{(0.020 \mathrm{GeV})^{2}}+\frac{\left(P_{m i s s}^{x}\right)^{2}+\left(P_{m i s s}^{y}\right)^{2}}{(0.014 \mathrm{GeV})^{2}}
$$



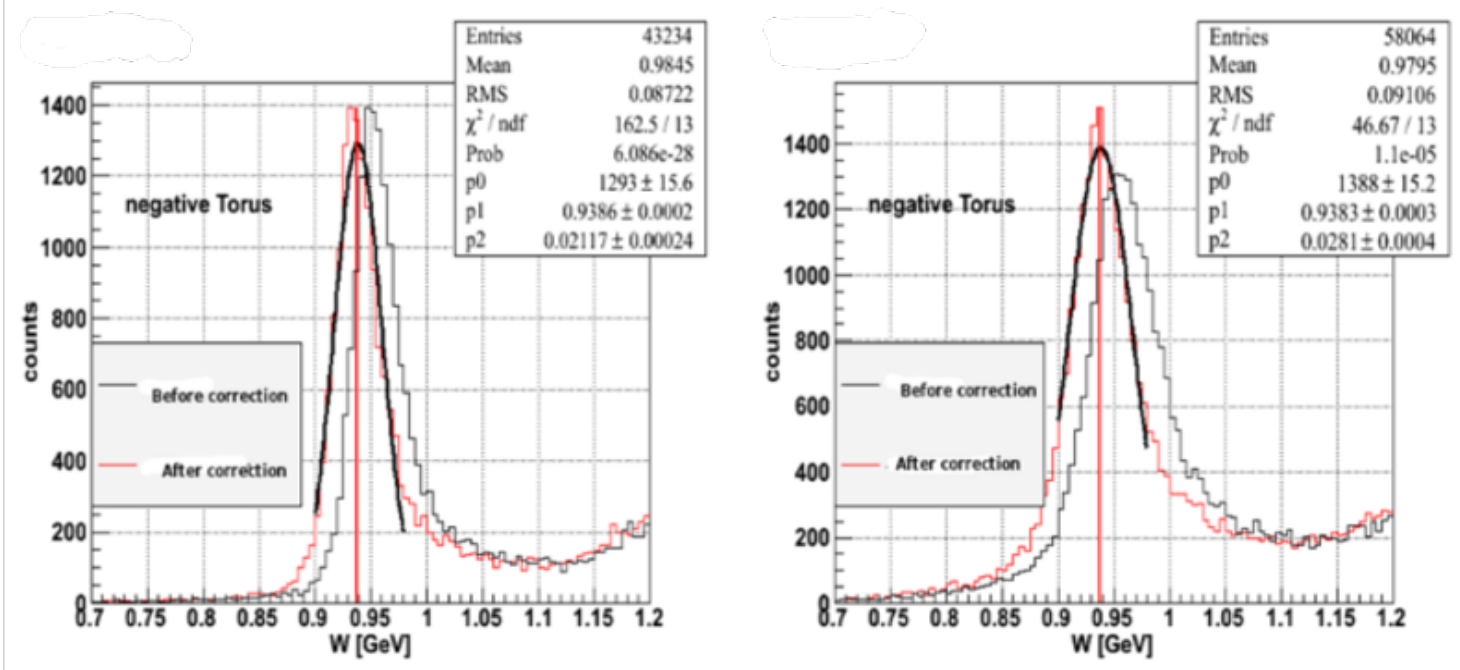

Figure 3.26: Comparison of the invariant mass distributions before and after applying momentum correction in CLAS sector 5 (left) and sector 6 (right). Black and red curves are the distributions before and after the momentum correction, respectively. The vertical line shows the proton mass $(0.938 \mathrm{GeV})$

After looping over all the selected events, 84 more terms, one for each of the 16 parameters, were added to the total $\chi^{2}$.

$$
\Delta \chi^{2}=\sum_{i=1}^{i=84} \frac{a_{i}^{2}}{\sigma_{i}^{2}\left(a_{i}\right)},
$$

where $a_{i}$ 's are the parameters of the fit. Once we obtained the optimized set of 84 parameters, we used Eq. 3.4. Eq. 3.5 and Eq. 3.8 to correct for the angle and momentum of each track and to recalculate the invariant mass distributions. Fig. 3.25 shows that $\phi$-dependence of the difference between the measured and calculated momenta disappears after our momentum correction. Also, Fig. 3.26 shows that the invariant mass distribution of elastic electron-proton scattering scattering shifts to the correct position as well and becomes slightly narrower. 


\section{Chapter 4}

\section{Data Analysis}

In order to analyze the data from this experiment, the novel analysis technique developed by Moteabbed et al. [49] was employed with some modifications. As discussed in Ref. [49], this analysis presented us with some unique challenges that are rare in other CLAS experiments. While precise energy of the incident beam is known in other CLAS experiments, the incident energy of the tertiary lepton beam particles in this experiment was unknown. However, the incident energy of the elastically scattered lepton can be reconstructed using elastic scattering kinematics. The usual CLAS charged particle identification schemes were not used in this analysis as the scheme generally requires data from the $\mathrm{CC}$ and $\mathrm{EC}$ in order to identify leptons. As already discussed, the CC and EC were not used in this experiment. Additionally, a leptoncharge independent analysis algorithm was required. To overcome these challenges, we required simultaneous detection of both the leptons $\left(e^{ \pm}\right)$and the protons $(p)$ in each event and measured both the momenta and scattering angles of the leptons and the protons. We employed this over-constrained kinematics to identify the elastic lepton-proton pair. This chapter will discuss the important steps involved in the data analysis.

\subsection{Elastic Event Selection}

The elastically scattered $e^{+} p$ and $e^{-} p$ events were the events of interest for this experiment. However, the collected trigger events contained not only the elastic events, but also a significant amount of inelastic background and accidental events. The elastic events were selected by making a series of cuts to remove the background events. We started the event selection process by skimming the data to keep only the events with 
at least two time-based tracks in the DC in opposite CLAS sectors. This skim got rid of significant amount of junk events from the data, reducing the data size by approximately $30 \%$. Ideally, the events with only two tracks should be taken. However, the event triggered by accidental hits could have more than two tracks per event. In that case, all possible combinations of the viable track pairs were formed by looping over all the time-based tracks in the event. The track pair was flagged as a viable pair if it satisfied the following preliminary cuts:

Good detector status: Both the tracks in the pair were required to have a detector hit in the drift chamber and the TOF paddle. Each track was also required to have a positive DC status (dc_stat>0), to select only the time-based tracks. Good charged pair: For the track pair to be a viable pair, one of the tracks must either be positive or negative $\left(e^{ \pm}\right)$while the other must be positive $(p)$.

Good vertex: To ensure that both tracks in the pair originate at the target, the reconstructed Z-vertex for both the tracks were required to be within the target length $\left(-45 \mathrm{~cm}<\mathrm{V}_{z}<-15 \mathrm{~cm}\right)$. Fig. 4.1 shows a typical reconstructed target vertex distribution for the lepton candidate and the proton candidate in the pair. The vertical lines show the extent of the target vertex cut. Fig. 4.2 shows a two-dimensional distribution of the azimuthal angle versus Z-vertex distributions of electron candidates in the viable -+ pair with both tracks having a good detector status.

The pairs that did not satisfy the above mentioned criteria were discarded. If none of the pairs of an event satisfied those criteria, the entire event was discarded to start over with the next event. The pair that satisfied above criteria was flagged as a possible elastic pair. Additional tests, based on kinematic cuts, were performed in order to decide whether the trial pair was indeed an elastic pair. If the pair is elastic, we can correctly identify the lepton and proton in the trial pair. Fig. 4.3 shows the analysis flow chart to identify the lepton and the proton in the trial pair. 

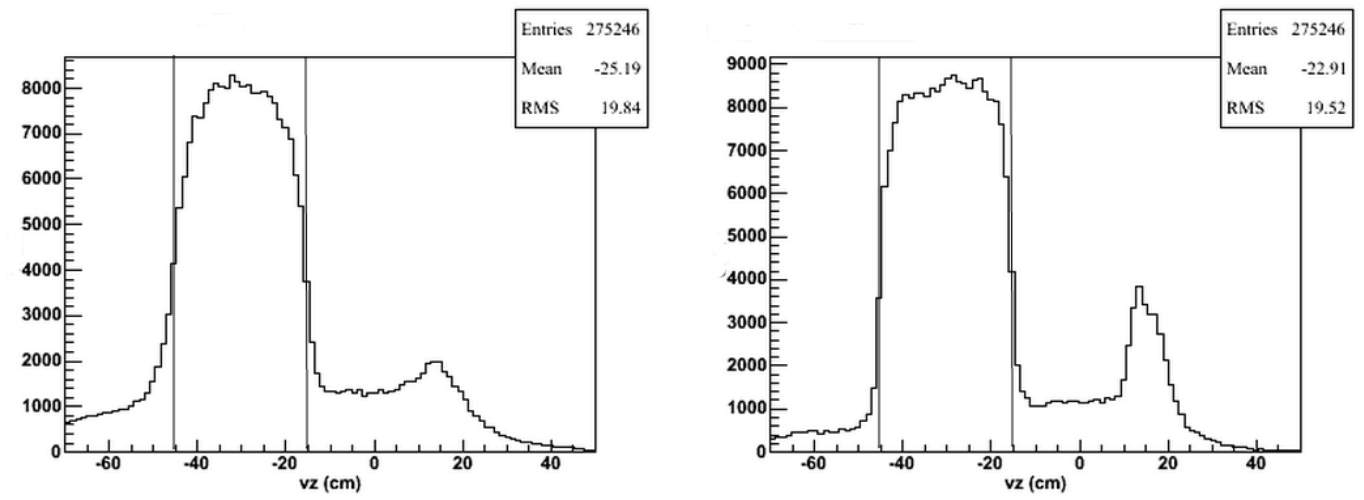

Figure 4.1: Reconstructed target vertex distribution for the electron candidate (left) and the proton candidate (right) in the -+ pair (dc_stat $>0)$. The vertical lines show the extent of the vertex cut.

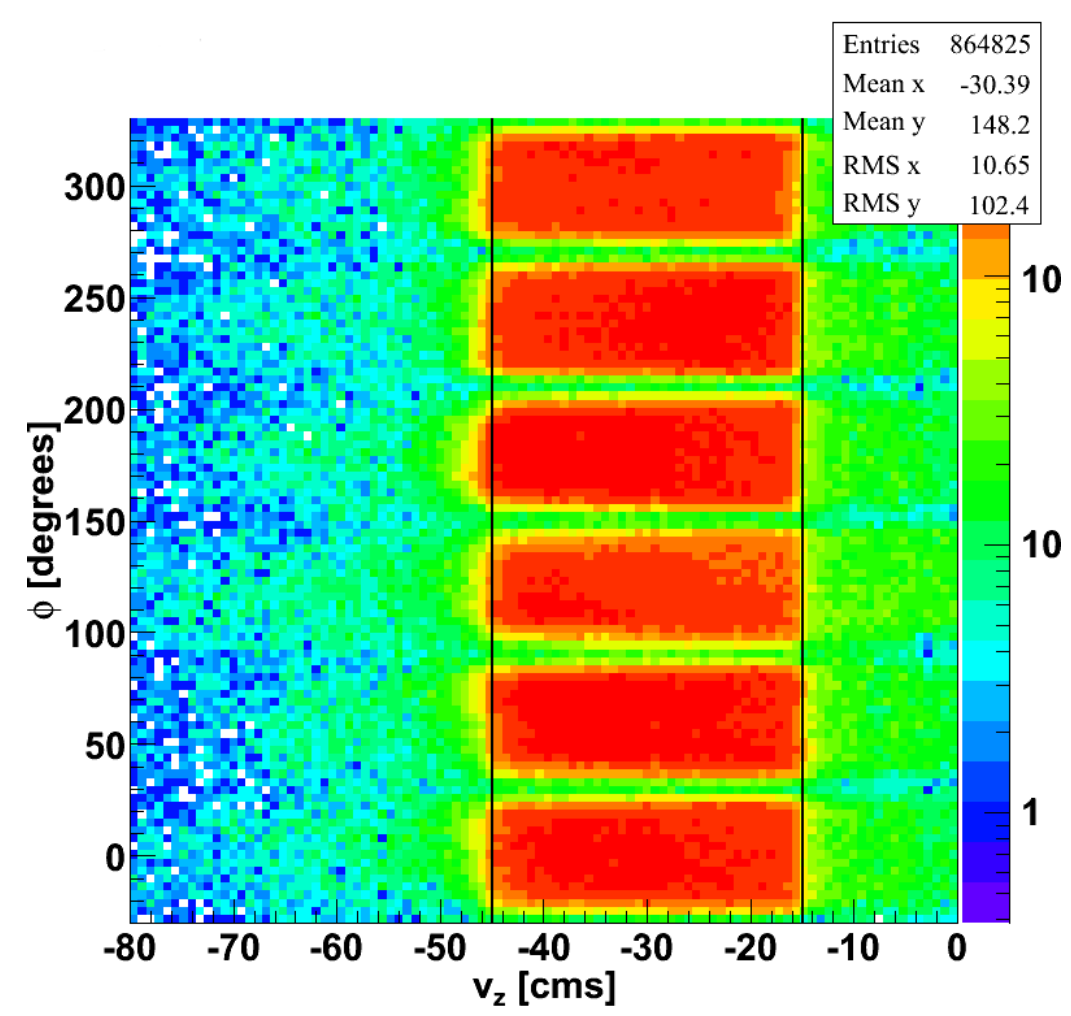

Figure 4.2: Azimuthal angle $(\phi)$ vs. $\mathrm{z}$-vertex $\left(\mathrm{v}_{z}\right)$ distribution for the electron candidate in the -+ pair. The vertical lines show the extent of the vertex cut.

The trial pair with a negatively-charged track and a positively-charged track was straightforward to analyze. The negatively-charged track represented an electron candidate while the positively charged track represented a proton candidate. The 


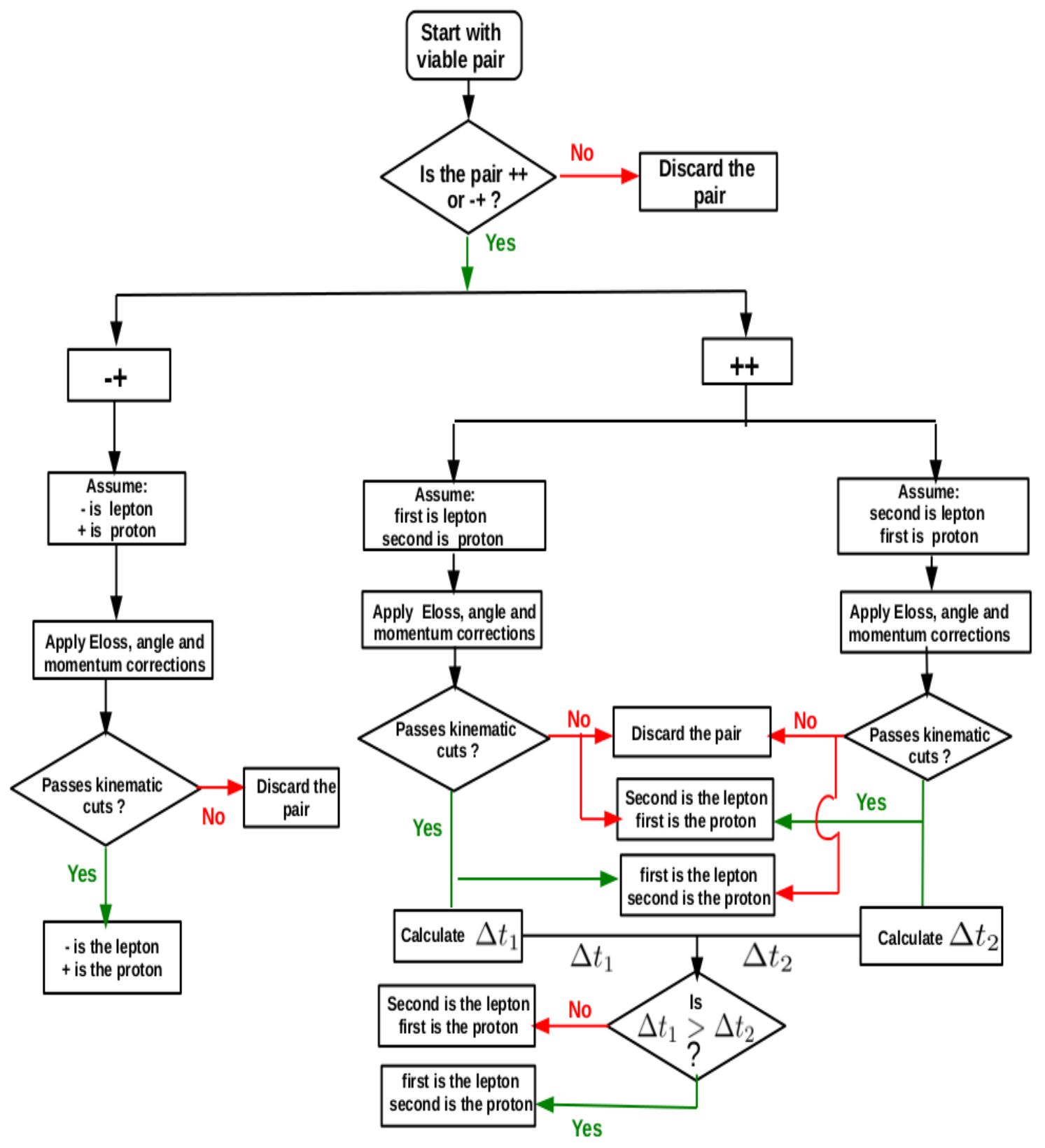

Figure 4.3: Analysis flow chart

energy loss and momentum corrections were applied accordingly. If the pair passed the elastic kinematic cuts described in the next section, it was flagged as the good elastic $e^{-} p$ pair. If the pair failed the elastic kinematic cuts, it was discarded from the analysis. 
Trial pairs with two positively charged tracks proved to be more complicated. To identify the positron and proton in a pair, we started with an assumption that one track in the pair represented a positron candidate while the other track represented a proton candidate. We then applied energy loss and momentum corrections accordingly. Next we checked whether the orientation passes the elastic kinematic cuts. Furthermore, we swapped the particle identities, applied energy loss and momentum corrections and checked whether the swapped orientation also passes the kinematic cuts. The lepton and the proton identities were then assigned based on which orientation passed the elastic kinematic cuts. If both orientations passed the kinematic cuts, the positron and proton identities were assigned based on which orientation yielded the minimum timing difference between the lepton and the proton candidates $(\Delta t=$ proton time - lepton time). It is to be noted that only a negligible fraction of events $\left(\sim 10^{-5}\right)$ had more than one pair passing all the kinematic cuts.

\subsubsection{Elastic Kinematic Cuts}

We employed over-constrained elastic scattering kinematics to cleanly select the elastic events and unambiguously identify the lepton and the proton in an event. Based on the elastic scattering kinematics, we chose to apply cuts on four different kinematic variables, which will be discussed in this section. To determine the width of the cut on each variable, the distribution (after applying cuts on the other three variables) was fitted with a Gaussian function. The cut width was then taken to be 3.5 standard deviations away from the mean on each side of the peak. We found that the mean and the standard deviation of the fits of each of these distributions slightly vary with the kinematic bins (discussed in section 4.3) and sectors. Hence, bin-wise and sector-wise cuts were applied to each of these distributions. 


\section{Co-planarity Cut}

Two elastically scattered particles were required to emerge back to back in the azimuthal direction. As shown in Fig. 4.4, the azimuthal angle difference between the lepton and the proton $\left(\Delta \phi=\phi_{l}-\phi_{p}\right)$ was peaked around $180^{\circ}$.
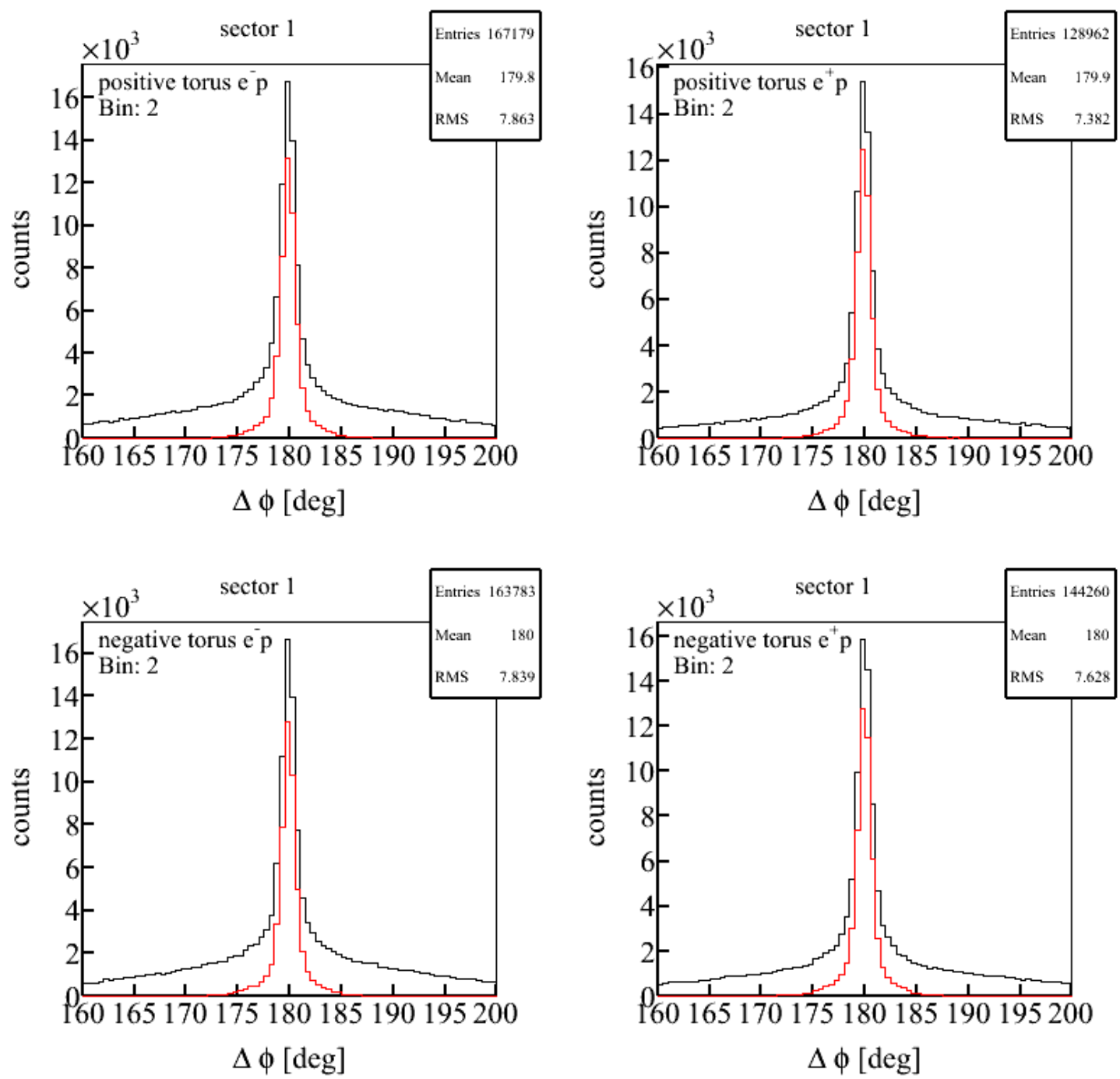

Figure 4.4: Distributions of the azimuthal angle difference between lepton and proton $(\Delta \phi)$ in a kinematic bin $\left(0.8<\varepsilon<0.945\right.$ and $\left.0.30<Q^{2} \leq 0.38\right)$ for event type and torus polarity as indicated. The lepton was detected in CLAS sector 1 . The black histogram is the data before applying any kinematic cuts and the red histogram is the data after applying all other kinematic cuts. 


\section{Lepton Energy Difference Cut}

The unknown beam energy of the incident tertiary electron-positron beam was reconstructed by using scattering angles of the lepton and the proton as,

$$
E_{\text {beam }}^{\text {angles }}=M\left(\frac{1}{\tan \left(\frac{\theta_{l}}{2}\right) \tan \theta_{p}}-1\right)
$$

where $\theta_{l}$ and $\theta_{p}$ are the lepton and proton scattering angles in the lab frame, respectively. Additionally, the incident lepton energy can also be reconstructed using both the scattering angles and the momenta of the lepton and the proton as,

$$
E_{\mathrm{beam}}^{\mathrm{mom}}=p_{l} \cos \theta_{l}+p_{p} \cos \theta_{p}
$$

where $p_{l}$ and $p_{p}$ are the lepton and the proton momenta. For ideal momentum and angle reconstruction, the two methods yield the same result. Hence, the difference between the incident energies calculated by using two methods sharply peaks around zero i.e.

$$
\Delta E_{\text {beam }}=E_{\text {beam }}^{\text {angles }}-E_{\text {beam }}^{\text {mom }} \approx 0 .
$$

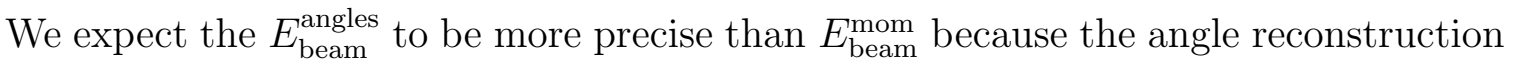
in CLAS has a better relative precision than the momentum reconstruction. In later calculations of kinematic quantities which involve beam energy, including $Q^{2}$ and $\varepsilon$, we use $E_{\text {beam }}^{\text {angles }}$.

Using elastic scattering kinematics, the energy of the scattered lepton can be calcu- 

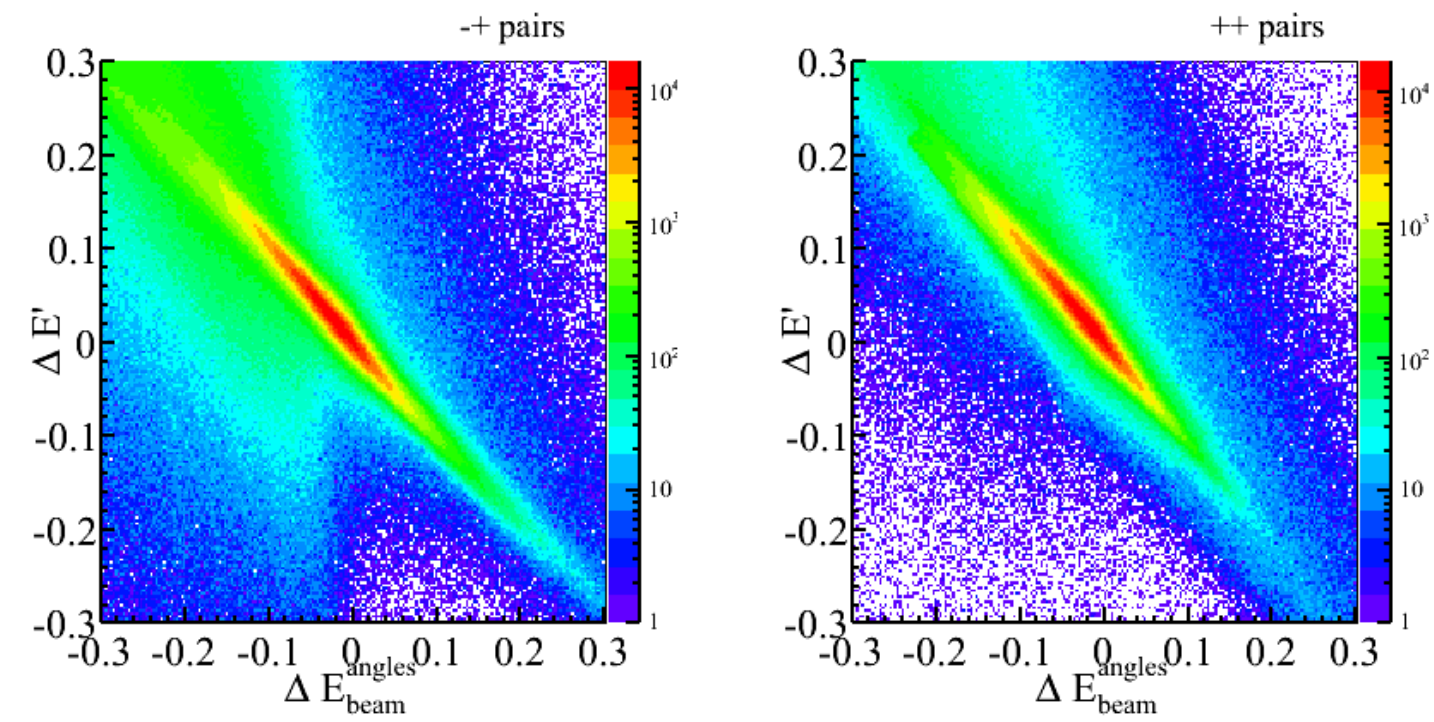

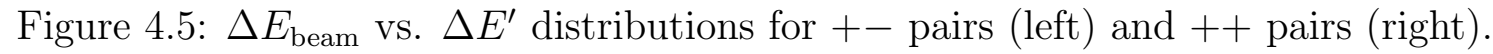

lated using the relation

$$
E_{\text {calc }}^{\prime}=\frac{E_{\text {beam }}^{\text {angles }} M}{1+E_{\text {beam }}^{\text {angles }}\left(1-\cos \theta_{l}\right)} .
$$

For ideal elastic scattering, the difference between the measured and the calculated momentum of the lepton,

$$
\Delta E^{\prime}=E_{\text {meas }}^{\prime}-E_{\text {calc }}^{\prime}
$$

is distributed around zero. Fig. 4.5 shows that $\Delta E_{\text {beam }}$ and $\Delta E^{\prime}$ are linearly correlated. We introduced a new set of cut variables on the basis of the correlation,

$$
\Delta E_{\text {beam }}+\Delta E^{\prime}, \quad \Delta E_{\text {beam }}-\Delta E^{\prime}
$$

Fig. 4.6 and Fig. 4.7 show the distributions of $\Delta E_{\text {beam }}+\Delta E^{\prime}$ and $\Delta E_{\text {beam }}-\Delta E^{\prime}$ in 

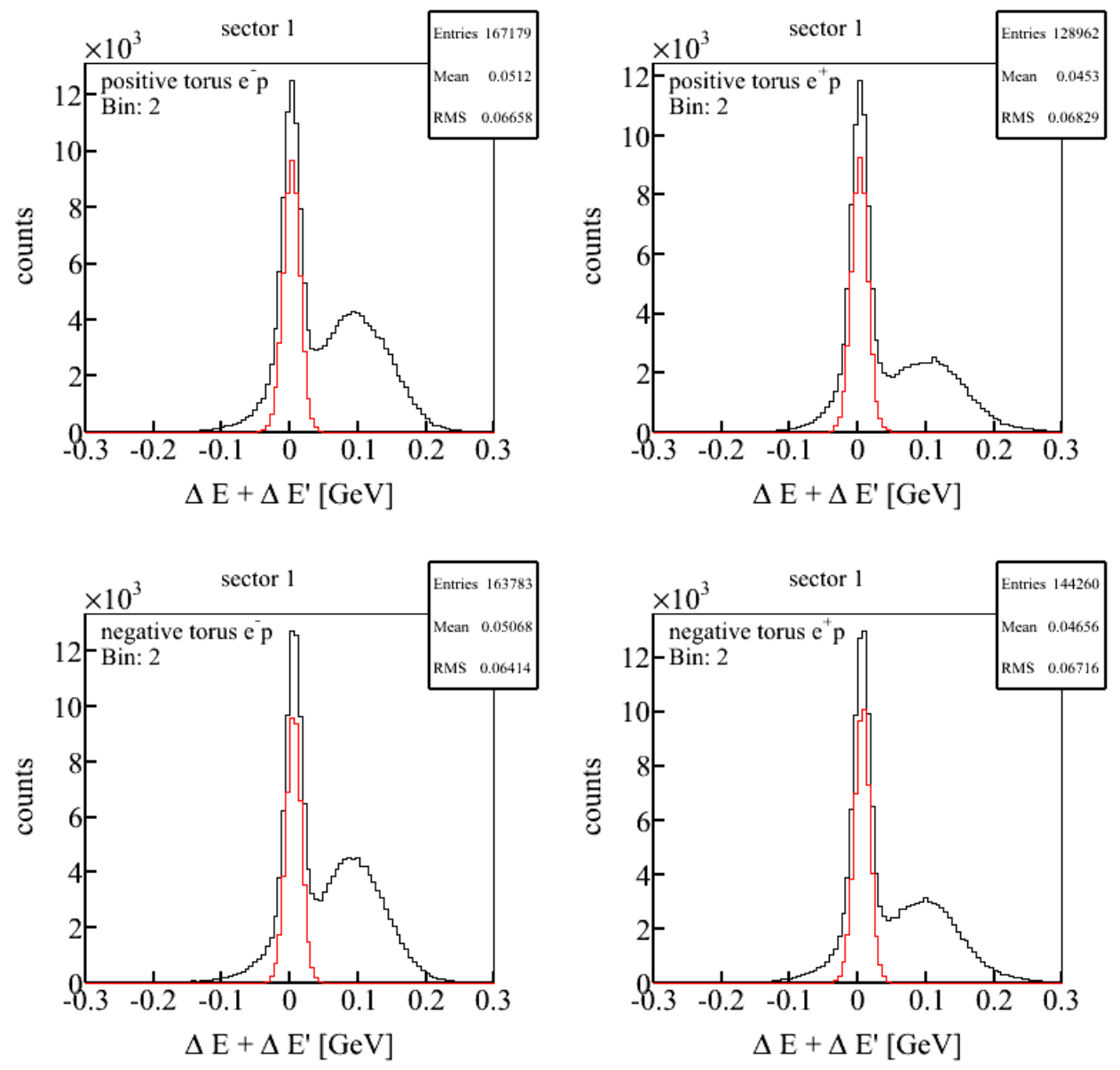

Figure 4.6: Distribution of $\Delta E_{\text {beam }}+\Delta E^{\prime}$ in a kinematic bin $(0.8<\varepsilon<0.945$ and $\left.0.30<Q^{2} \leq 0.38\right)$ for event type and torus polarity as indicated. The lepton was detected in CLAS sector 1. The black histogram is the data before applying any kinematic cuts and the red histogram is the data after applying all other kinematic cuts.

CLAS sector 1 and one of our kinematic bins. As expected, both of these distributions are sharply peaked at zero.

\section{Proton Momentum Difference Cut}

The momentum of the recoil proton was calculated from the scattering angles of the lepton and the proton using Eq. 4.7. This was compared with the measured 

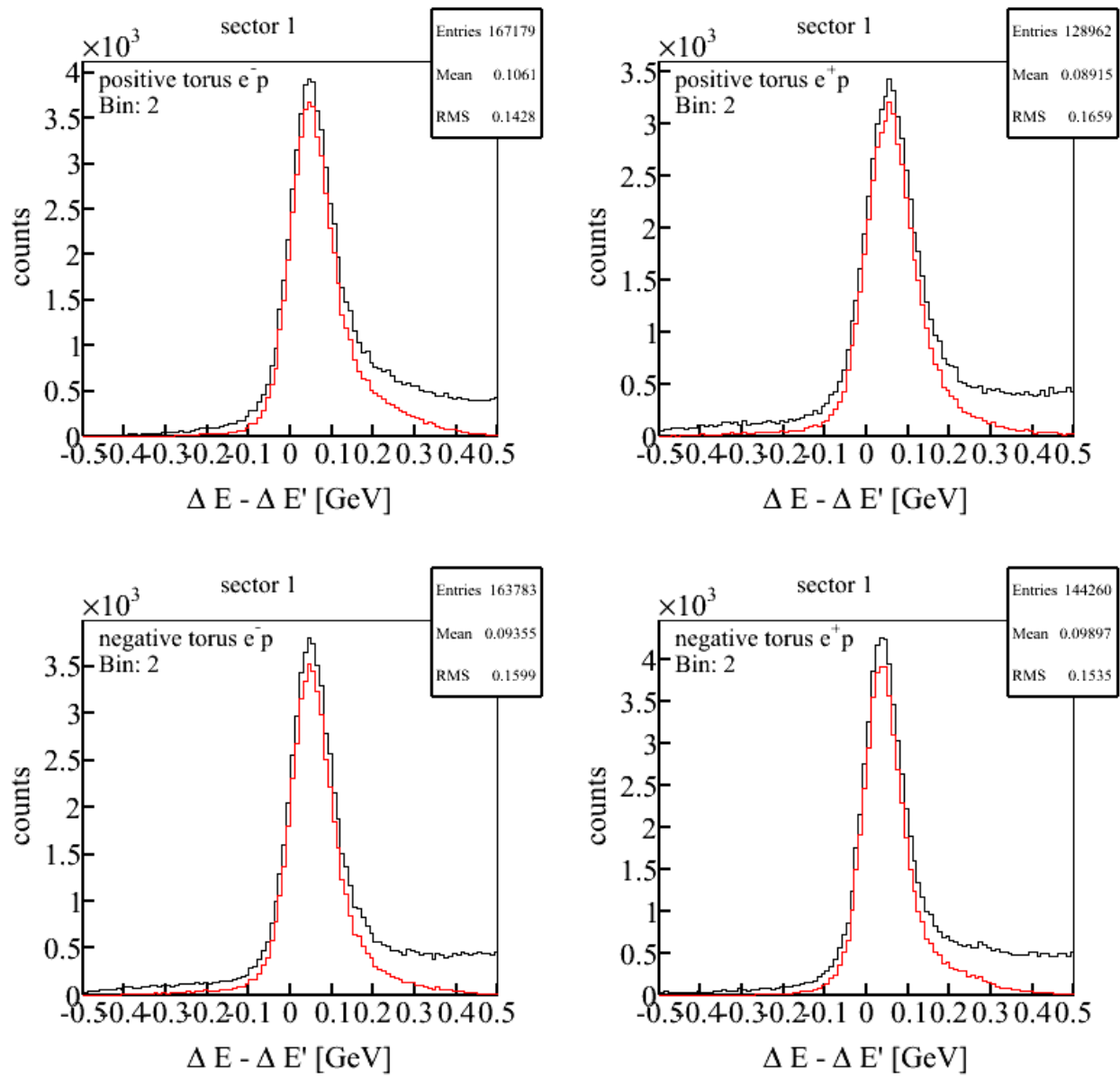

Figure 4.7: Distributions of $\Delta E_{\text {beam }}-\Delta E^{\prime}$ in a kinematic bin $(0.8<\varepsilon<0.945$ and $\left.0.30<Q^{2} \leq 0.38\right)$ for event type and torus polarity as indicated. The lepton was detected in CLAS sector 1. The black histogram is the data before applying any kinematic cuts and the red histogram is the data after applying all other kinematic cuts.

momentum of the proton $p_{p}^{\text {meas }}$

$$
p_{p}^{\text {calc }}=\frac{p_{l} \sin \theta_{l}}{\sin \theta_{p}}, \quad \Delta p_{p}=p_{p}^{\text {meas }}-p_{p}^{\text {calc }}
$$

Fig. 4.8 shows the distribution for the difference between the measured and calculated momenta of the proton in one CLAS sector for one of our kinematic bins. As expected, the distribution is sharply peaked at around zero. 

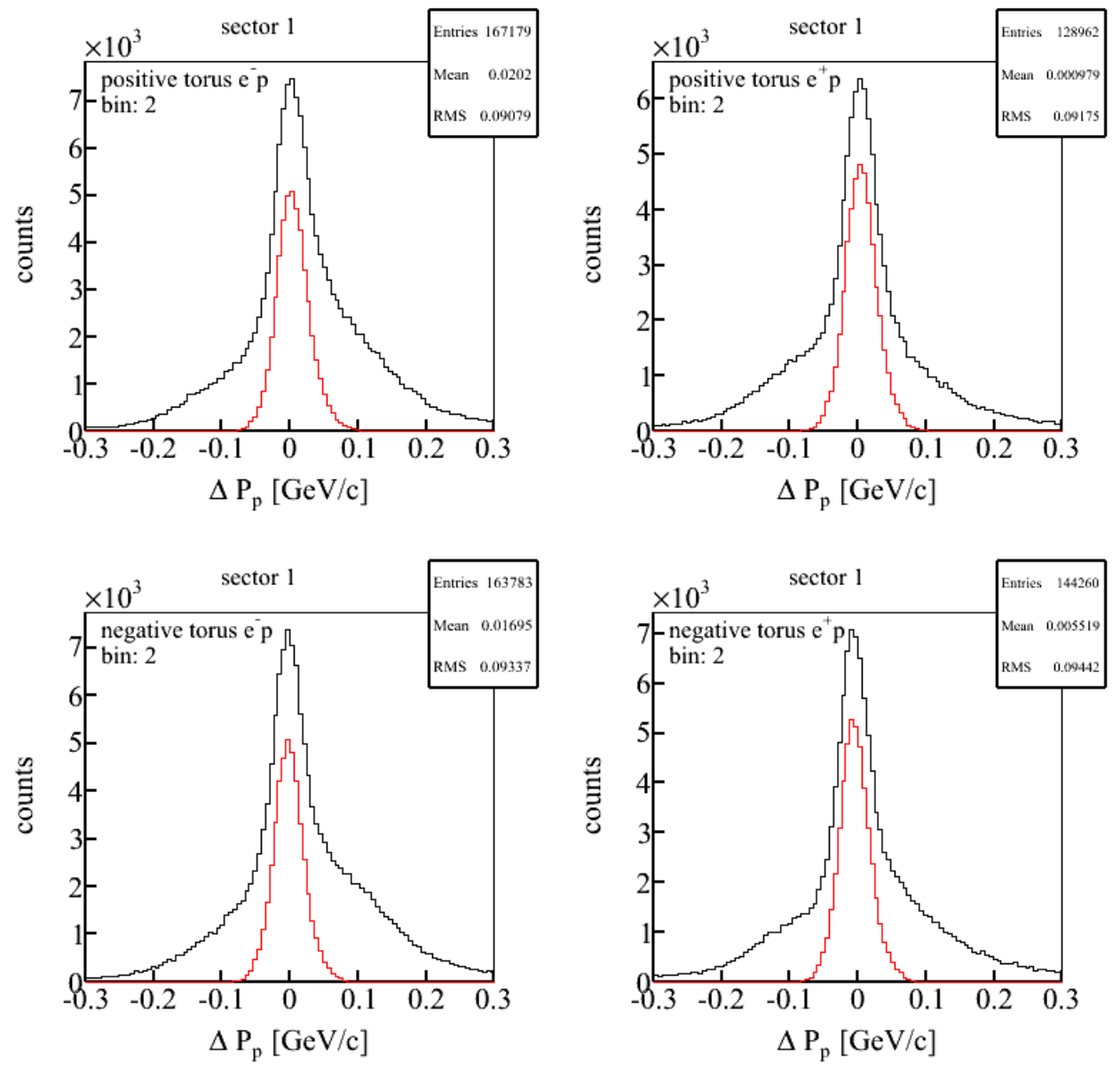

Figure 4.8: Distributions of $\Delta p_{\mathrm{p}}$ in a kinematic bin $(0.8<\varepsilon<0.945$ and $0.30<$ $\left.Q^{2} \leq 0.38\right)$ for event type and torus polarity as indicated. The lepton was detected in CLAS sector 1. The black histogram is the data before applying any kinematic cuts and the red histogram is the data after applying all other kinematic cuts.

\section{Fiducial Cuts}

It was crucial for this experiment to have the same geometrical acceptances for both in-bending and out-bending particles for both torus magnet polarities. In order to select the regions of CLAS with uniform detection efficiency for both lepton species, momentum and angle dependent fiducial cuts were applied. Any region of CLAS where the particle detection efficiency changes rapidly were removed by applying the fiducial cuts. In order to determine the optimum fiducial cuts, the momenta of the 

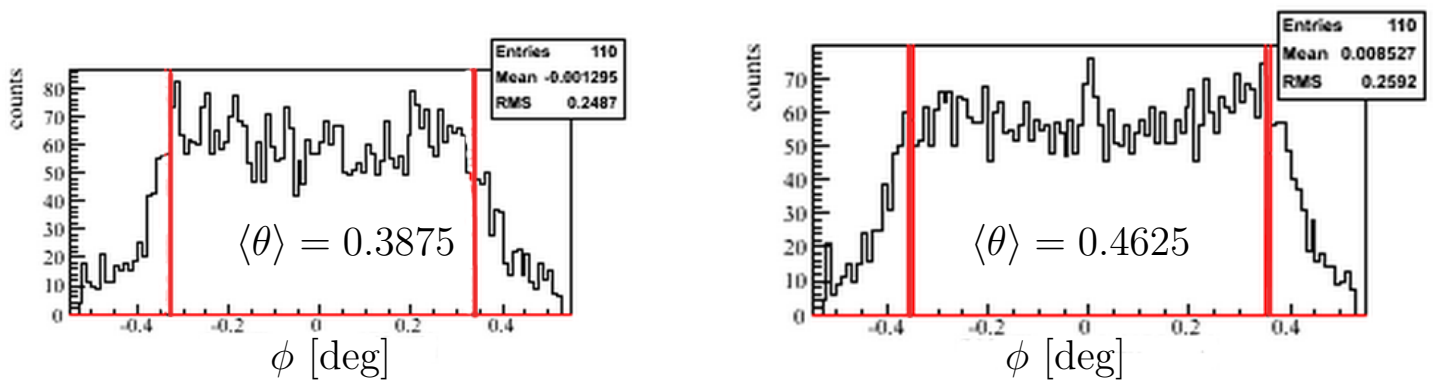

Figure 4.9: The azimuthal angle $(\phi)$ distributions at $\langle\theta\rangle=0.3875$ (left) and $\langle\theta\rangle=$ 0.4625 for the bin where $0.2<p<0.3 \mathrm{GeV} / \mathrm{c}$. The red vertical lines show the applied cuts.

reconstructed tracks were divided into several bins. In the given momentum bin, different slices of scattering angles $(\theta)$ were projected into one-dimensional azimuthal angle $(\phi)$ distributions. Fig. 4.9 shows representative $\phi$-distributions in two $\theta$-bins where $0.2<p<0.3 \mathrm{GeV} / \mathrm{c}$. A cut indicated by red vertical lines was applied to remove the region of $\phi$ where the distribution was rapidly varying. We then used functional forms of the fiducial cuts applied by the CLAS g13 collaboration 65] with the fit parameters adjusted using our data. Fig. 4.10 and Fig. 4.11 show two dimensional $\theta-\phi$ distributions for in-bending and out-bending tracks respectively at several momentum slices. The fiducial cuts are drawn in black. Data from all CLAS sectors are combined to produce these plots. Fig. 4.10 shows that the angular acceptance of in-benders are momentum-dependent at small $\theta$. Hence, we added a momentum dependent $\theta$-shift to the original $\theta$ for the in-bending tracks, i.e, $\theta^{\prime}=\theta+\theta$ shift with,

$$
\theta \text {-shift }=0.1913+\frac{0.2012 \mathrm{GeV}}{(p+0.2997 \mathrm{GeV})}
$$

This value of $\theta^{\prime}$ is then used in Eq. 4.9) and (4.10). See Fig. 4.12. The fiducial cuts used for in-bending and out-bending tracks are $|\phi|<\phi_{\max }$ where, for in-bending 

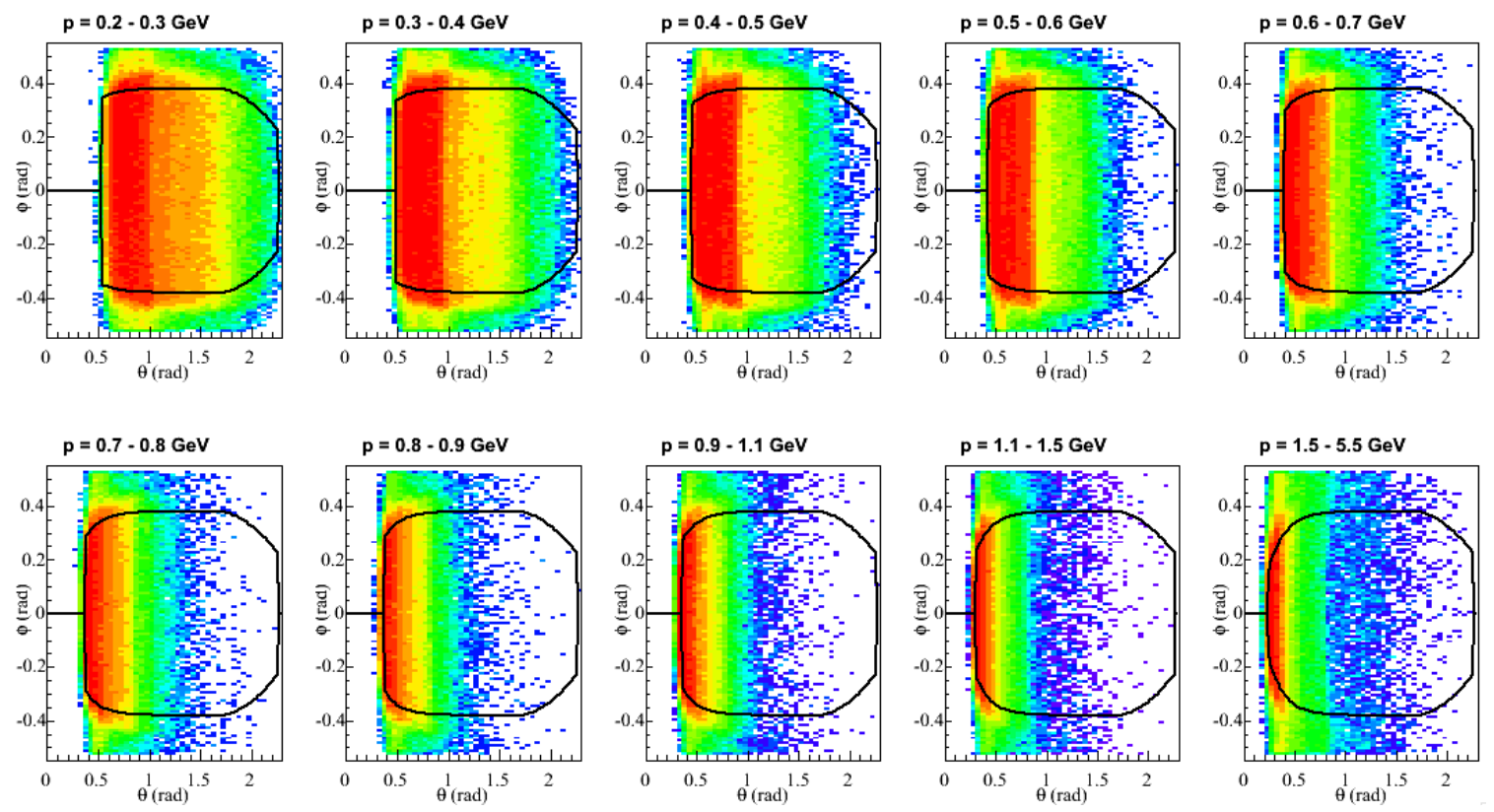

Figure 4.10: The distribution of reconstructed electron tracks as a function of $\theta$ and $\phi$ for positive torus (in-bending tracks) for all sectors for momentum bins indicated on the top of each plot. Fiducial cuts are drawn in black.
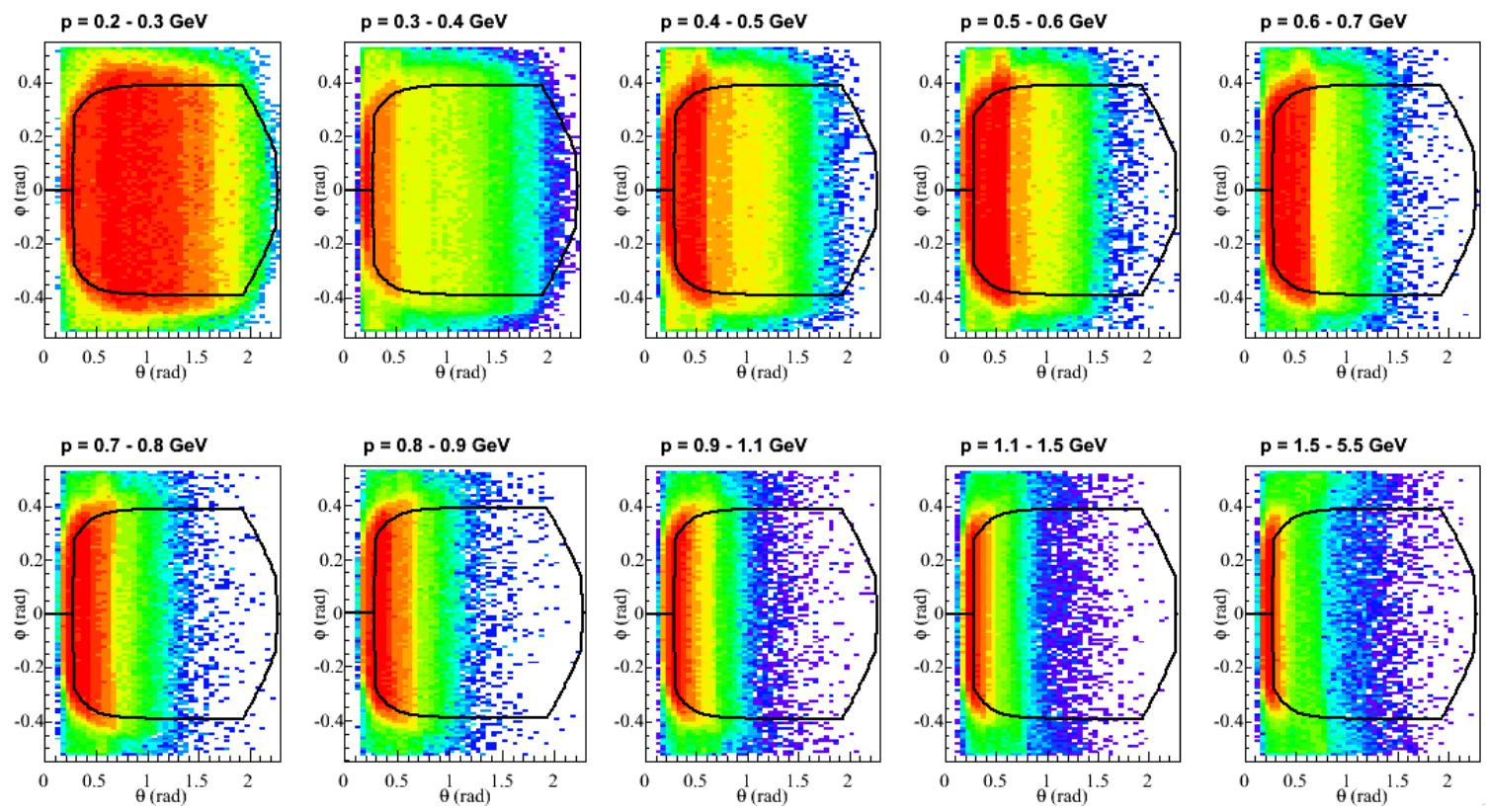

Figure 4.11: The distribution of reconstructed electron tracks as a function of $\theta$ and $\phi$ for negative torus (out-bending tracks) for all sectors for momentum bins indicated on the top of each plot. Fiducial cuts are drawn in black. 


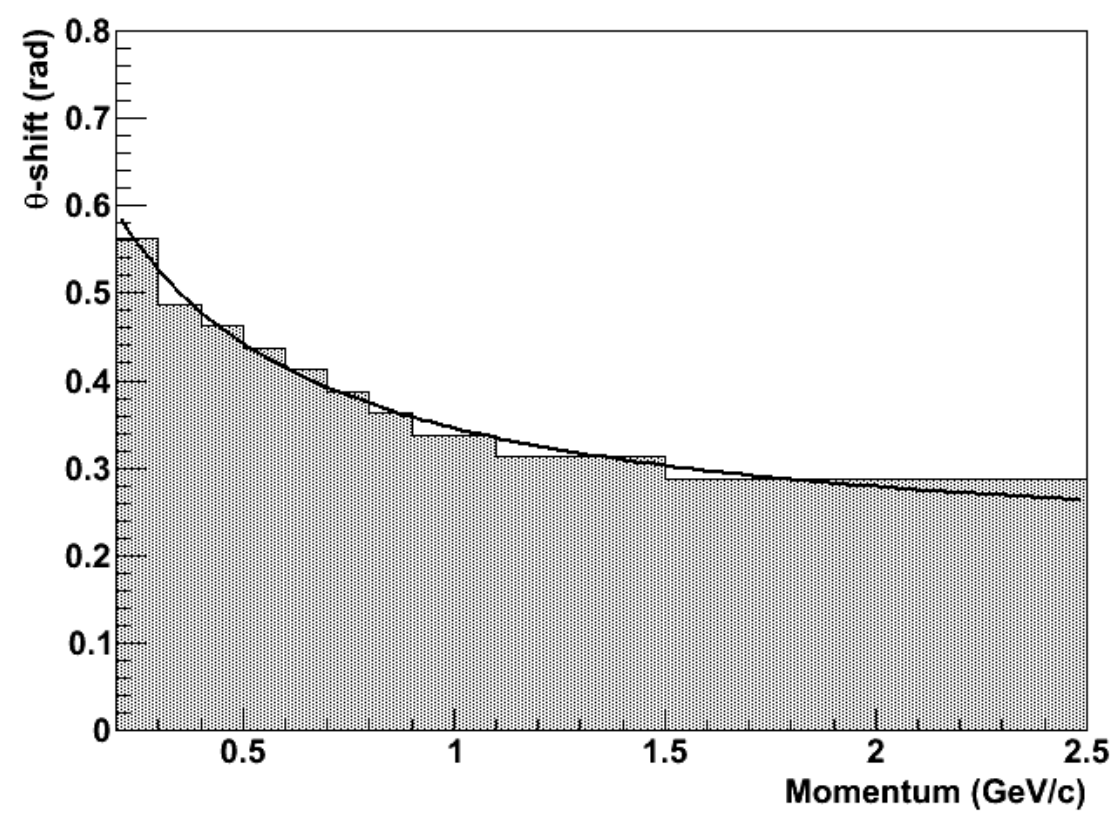

Figure 4.12: $\theta$-shift versus momentum of reconstructed electrons for positive torus magnet setting (in-bending tracks). The fit to this distribution was used for determining the shift of fiducial cuts for in-bending tracks.

tracks (with $\phi_{\max }$ in radians),

$$
\phi_{\max }=\left\{\begin{array}{lc}
0.38\left(1-e^{-6.5 \theta^{\prime}+1.0}\right), & 0.237<\theta^{\prime}<1.7 \mathrm{rad} \\
-0.4 \theta^{\prime 2}+1.07 \theta^{\prime}-0.674, & \theta^{\prime}>1.7 \mathrm{rad}
\end{array}\right\}
$$

and for out-bending tracks,

$$
\phi_{\max }=\left\{\begin{array}{lc}
0.39\left(1-e^{-6.5 \theta^{\prime}+0.6}\right), & 0.187<\theta^{\prime}<1.92 \mathrm{rad} \\
-0.44 \theta^{\prime 2}+1.07 \theta^{\prime}-0.042384, & \theta^{\prime}>1.92 \mathrm{rad}
\end{array}\right\}
$$

Fig. 4.10 and Fig. 4.11 show that the fiducial cuts are within the regions of uniform acceptance for both in-bending and out-bending leptons. 

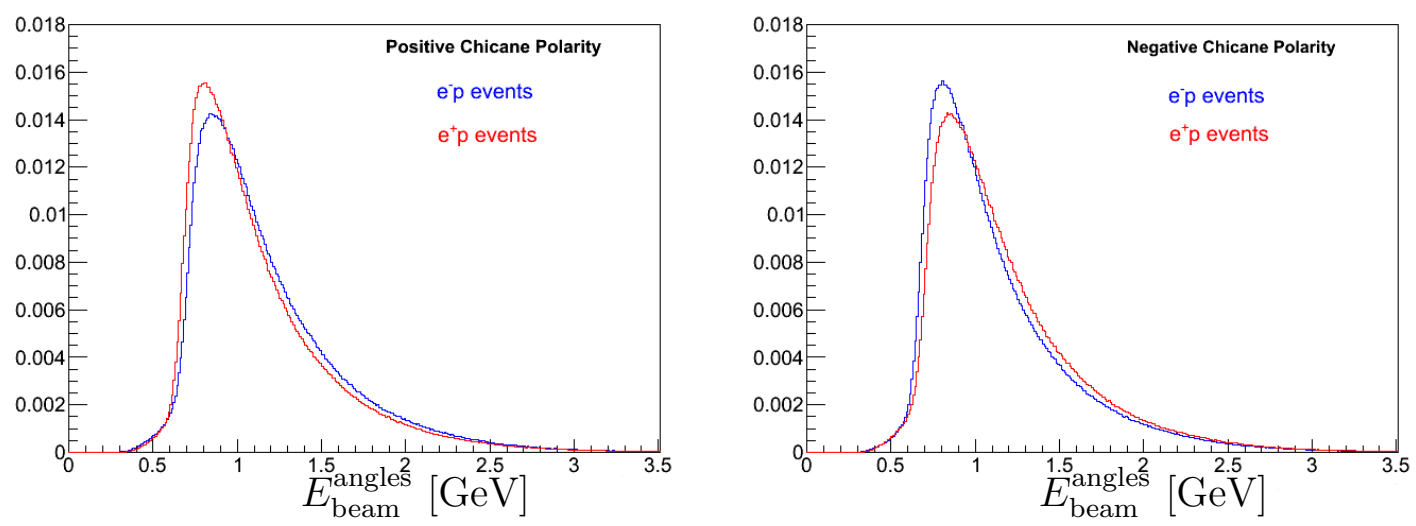

Figure 4.13: Reconstructed incident beam energy distributions of elastically scattered events using scattering angles $\left(E_{\text {beam }}^{\text {angles }}\right)$ for positive (left) and negative (right) chicane magnet polarity. The area of the histograms are normalized to unity.

\subsubsection{Beam Energy Distribution}

Fig. 4.13 shows the incident energy distributions for both lepton species for both positive and negative chicane polarities. The incident energy in these distributions were calculated using the scattering angles of the lepton and the proton. Furthermore, it shows that the distributions are slightly different for different chicane polarities due to a left-right asymmetry in the chicane. This observation is consistent with the lepton incident energy as measured in the TPECal as discussed in section 4.6. However, the incident energy distribution for $e^{-} p$ events in one chicane polarity is similar to the incident energy distribution for $e^{+} p$ events in the opposite chicane polarity and vice versa. As seen in Fig. 4.14, the incident energy distributions for both $e^{+} p$ and $e^{-} p$ events are similar when data from both chicane polarities are combined. This clearly demonstrates the benefits of taking data in both chicane polarities in order to cancel any chicane-dependent asymmetries. This will be discussed further in Sect. 4.5. To avoid very low energy leptons, the incident energy was required to be greater than $0.85 \mathrm{GeV}$. 


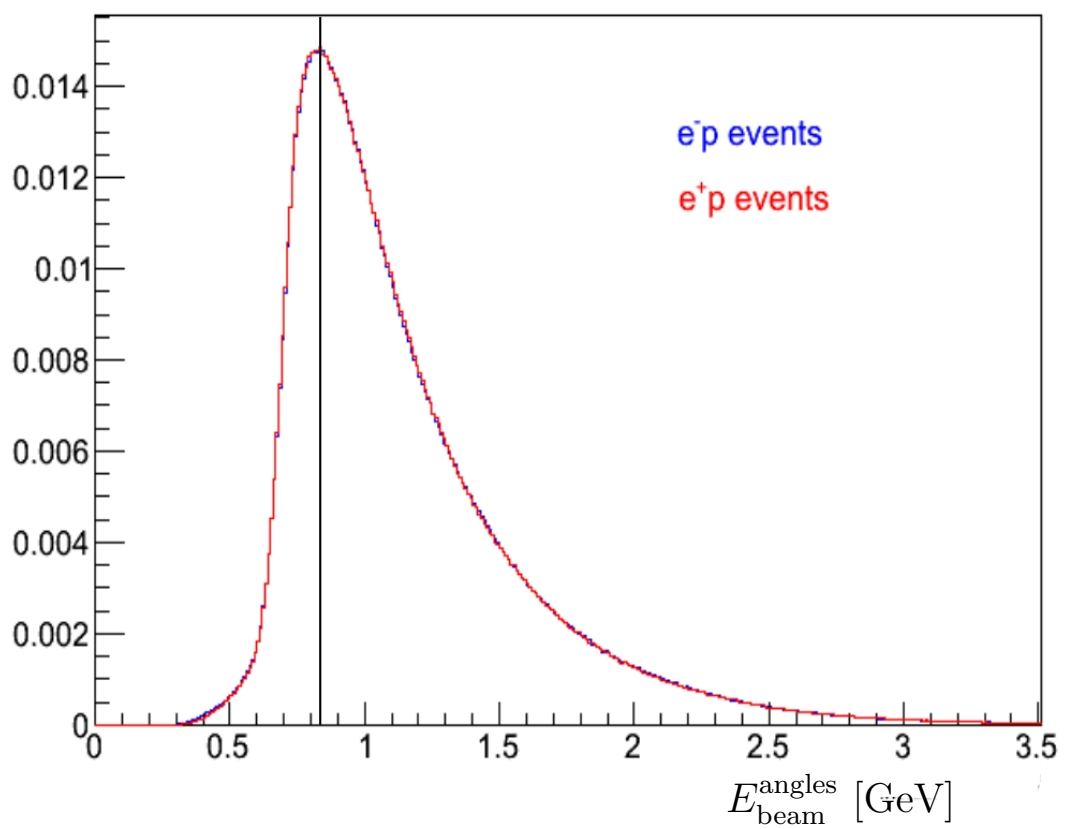

Figure 4.14: Reconstructed incident beam energy distributions of elastically scattered events using scattering angles $\left(E_{\text {beam }}^{\text {angles }}\right)$. The vertical line shows the applied beam energy cut. The histograms are normalized to unity.

\subsection{Dead Detector Corrections}

Dead and inefficient detector components could create a false asymmetry and their effect needs to be considered. To account for this effect, the following two procedures were implemented.

\subsubsection{Inefficient Detector Removal}

The inefficient detector channels are defined to be either dead, broken, or inefficient detector components. For example, the forward region $\left(8^{\circ}<\theta<45^{\circ}\right)$ in the sector three drift chamber and EC had many such regions. To minimize any systematic bias because of such regions, any event with either a lepton or proton going forward in sector three was discarded in all torus and chicane configurations. Similarly, some 
TOF scintillator paddles also had significantly fewer counts than their neighboring paddles. The paddles that had significantly fewer counts for the entire momentum range were marked as inefficient. To minimize any systematic bias in lepton detection efficiency due to the defective paddles, they were completely removed from the analysis. Table 4.1 lists the identified bad paddles in each sector.

Table 4.1: Summary of the Bad TOF paddles

\begin{tabular}{cc}
\hline \hline Sector & TOF paddle \\
\hline 1 & 23,34 \\
2 & 26 \\
3 & 16,23 \\
4 & $16,23,29$ \\
5 & $8,12,23,30$ \\
6 & 29 \\
\hline \hline
\end{tabular}

\subsubsection{Swimming Corrections}

This experiment relies on the fact that the electron and the positron acceptances are exactly the same. The acceptances of the two lepton species were matched by implementing a "swimming" algorithm, which can trace the particle trajectories through the CLAS geometry and magnetic field. Taking the vertex position of the particle and the three components $\left(p_{x}, p_{y}, p_{z}\right)$ of three-momentum $(p)$ as an input, the algorithm predicts the hit positions on the detector planes. These hit positions can be converted into sectors and paddle numbers as required. For the given lepton in an event, the algorithm generates a conjugate lepton at the same vertex with the same momentum and scattering angle as the original lepton.

Fig. 4.15 shows a pictorial display of the swimming algorithm. For each elastic $e^{-} p$ $\left(e^{+} p\right)$ event detected in CLAS, the swimming algorithm generates a conjugate $e^{+}\left(e^{-}\right)$ event with the same momentum and vertex. Both the lepton and its conjugate are 


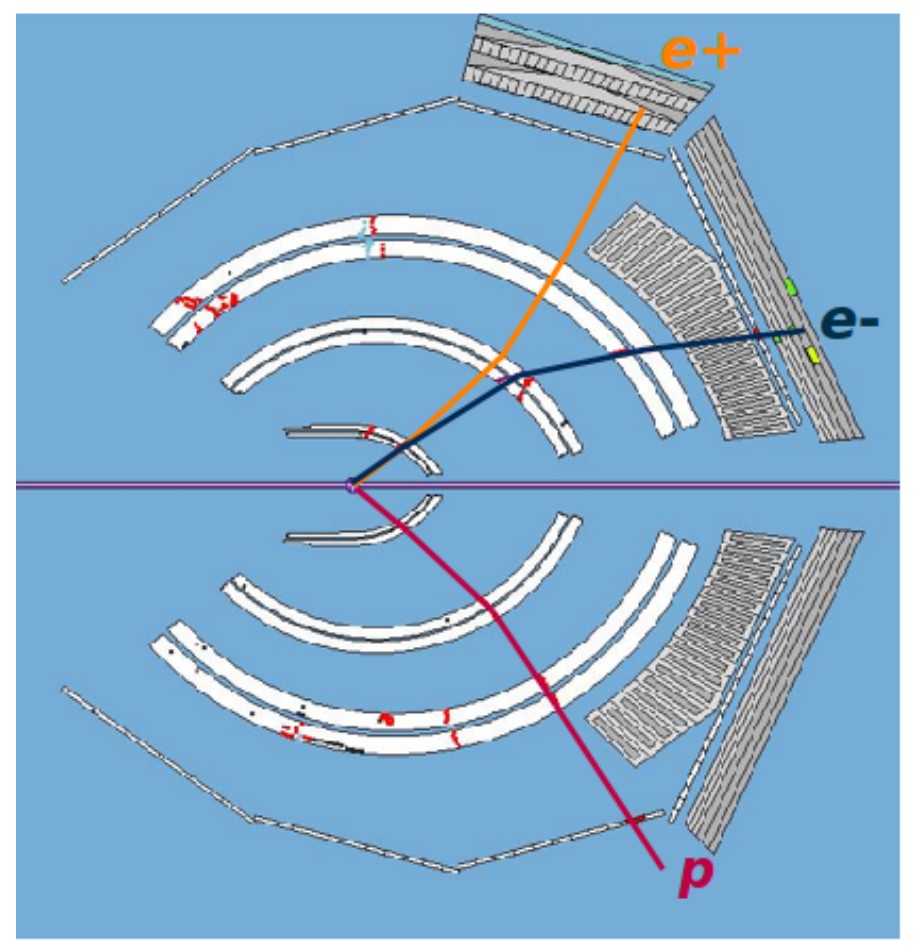

Figure 4.15: Swimming of the detected event through CLAS

then swum through the CLAS geometry and magnetic field to predict the sector and paddle hit by the lepton and its conjugate. The event is accepted as long as both the lepton and its conjugate are within the fiducial acceptance region of CLAS and both hit a good scintillator paddle. Otherwise, the event was rejected. The swimming algorithm takes into account the effect of the CLAS mini-torus, whose polarity was fixed throughout the experiment.

\subsection{Kinematic Coverage and Bin Selection}

Fig. 4.16 shows the kinematic coverage of the experiment in the $Q^{2}$ versus $\varepsilon$ distribution for the events that pass all the above mentioned cuts. Since the distributions were the same for both positive and negative chicane polarities, the data from both polarities were combined to produce these plots. The holes in the kinematic coverage at around $\varepsilon \approx 0.6$ shown by red triangles are the trigger holes. These occur because we required at least one particle to hit the forward TOF panel $\left(\theta<45^{\circ}\right)$ and $\mathrm{EC}$ on 

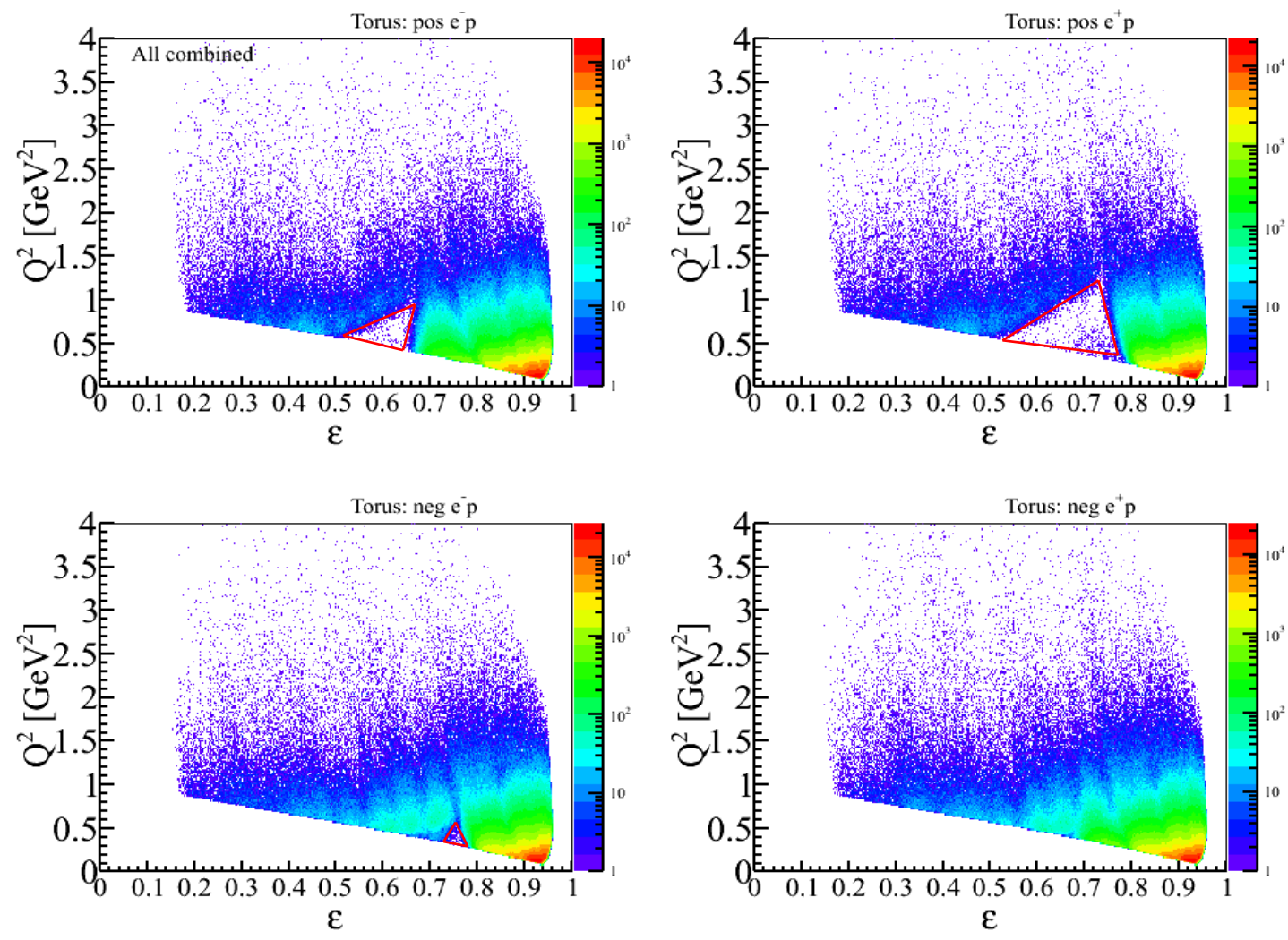

Figure 4.16: $Q^{2}$ vs. $\varepsilon$ distributions. Top-left: Positive torus $e^{-} p$ events; Top-right: Positive torus $e^{+} p$ events; Bottom-left: Negative torus $e^{-} p$ events; Bottom-right: Negative torus $e^{+} p$. The red hollow triangles show the trigger holes. Note that data from all CLAS sectors are combined.

the same sector. For a certain range of kinematics that depend on the torus polarity, both the lepton and the proton miss the forward angle TOF panel. It is clear that the trigger hole is the most prominent for $e^{+} p$ events in positive CLAS torus polarity. It can also be seen that the elastically scattered events are largely concentrated at low $Q^{2}$ and high $\varepsilon$ as expected since the cross section drops rapidly with $Q^{2}\left(\sigma \propto \frac{1}{Q^{4}}\right)$.

Fig. 4.17 shows the data binning schemes adopted in the data analysis. The bins are overlaid on the $Q^{2}$ versus $\varepsilon$ distribution of $e^{+} p$ events in positive torus polarity. The bins shown in the top two plots are the main focus of this dissertation while the bins shown in bottom two plots were analyzed by another collaborator [66]. The 

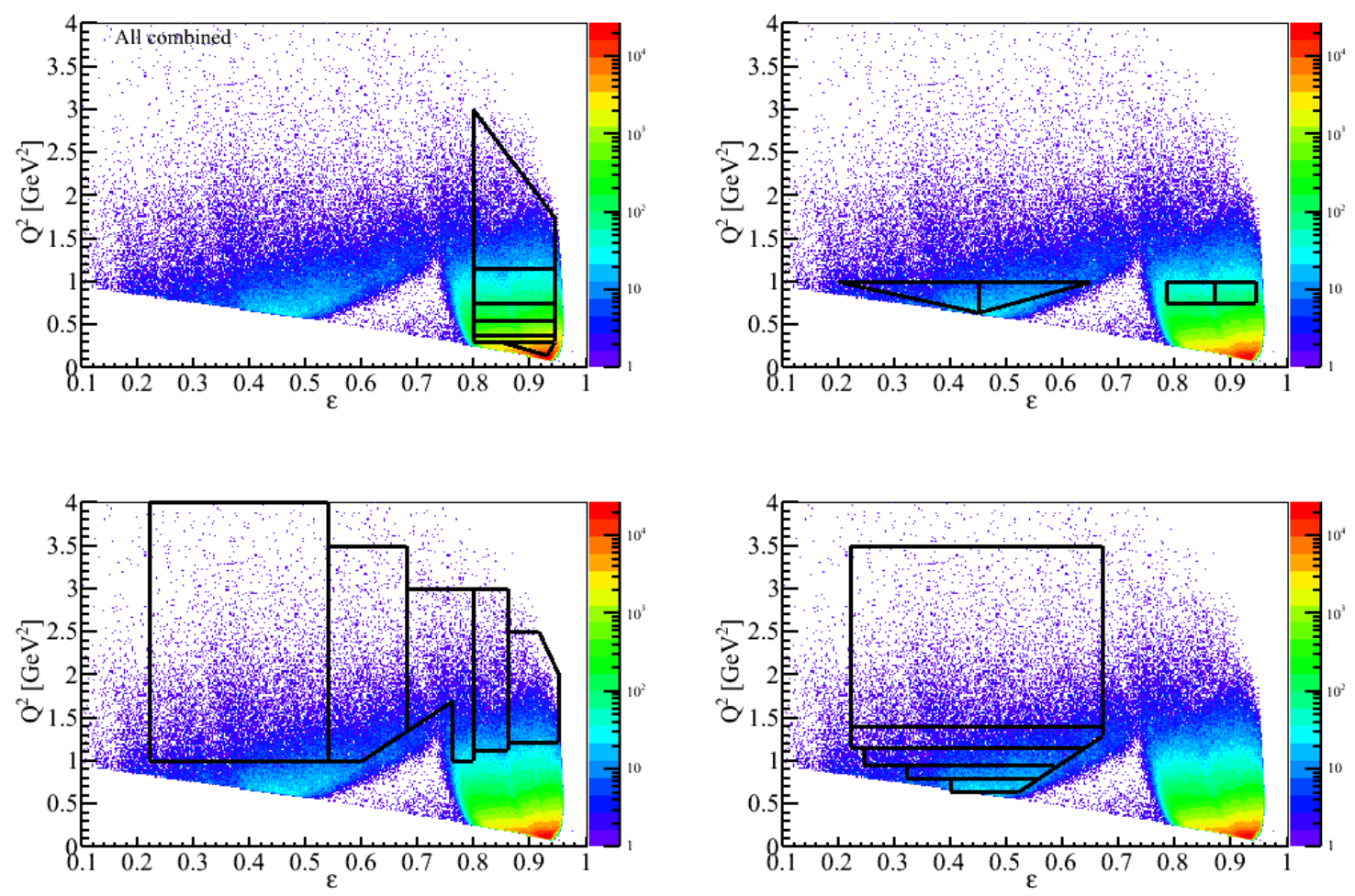

Figure 4.17: Binning schemes adopted in data analysis. Top-left: 6 bins in high $\varepsilon$; Top-right: 4 bins in low $Q^{2}$, Bottom-left: 5 bins in high $Q^{2}$; Bottom-right: 5 bins in low $\varepsilon$. The bins in the top-left and top-right are the main focus of this dissertation. The bins are overlaid on $Q^{2}$ versus $\varepsilon$ distributions for the $e^{+} p$ events in positive torus polarity where the trigger hole is most prominent.

binning scheme avoids kinematic holes and the edges of the distributions where the acceptance changes rapidly. The results presented in this dissertation cover 6 bins in $Q^{2}$ at high $\varepsilon(\varepsilon>0.8)$ and 4 bins in $\varepsilon$ at $\left.\left\langle Q^{2}\right\rangle=0.85 \mathrm{GeV}^{2}\right)$. The primary goal of adopting such binning was to investigate the $Q^{2}$ dependence of the TPE effect at high $\varepsilon(\varepsilon>0.8)$ and the $\varepsilon$ dependence of the effect at low $Q^{2}$. The average values of $Q^{2}$ and $\varepsilon$ for these bins are summarized in Table 4.2 .

\subsection{Background Subtraction}

Even after applying all the event selection cuts discussed in the previous sections, background can still remain in some bins. Fig. 4.18 demonstrates that the background 
Table 4.2: Average $\left\langle Q^{2}\right\rangle$ and $\langle\varepsilon\rangle$ for the binned data

\begin{tabular}{cccc}
\hline & bin & $\left\langle Q^{2}\right\rangle\left(\mathrm{GeV}^{2}\right)$ & $\langle\varepsilon\rangle$ \\
\hline \hline & 1 & 0.232 & 0.915 \\
High $\varepsilon$ & 2 & 0.336 & 0.887 \\
& 3 & 0.449 & 0.886 \\
& 4 & 0.632 & 0.885 \\
& 5 & 0.893 & 0.882 \\
& 6 & 1.415 & 0.874 \\
\hline \hline & 1 & 0.389 & 0.844 \\
Low $Q^{2}$ & 2 & 0.511 & 0.859 \\
& 3 & 0.831 & 0.849 \\
& 4 & 0.908 & 0.852 \\
\hline
\end{tabular}

is negligible at high $\epsilon$ bins. However, a small background still remains at the lower $\varepsilon$ bins (see Fig. 4.19). Any charge asymmetric background under the peaks for $e^{+} p$ and $e^{-} p$ events can introduce a false cross-section asymmetry. In order to precisely determine the cross-section ratio, it is crucial to correctly determine the background and subtract it from the signal.

We performed an in-depth analysis of the background in the bins, where the background was significant [60]. We found that the background can be correctly sampled by fitting the tails of the $\Delta \phi\left(=\phi_{l}-\phi_{p}\right)$ distribution, for the events that pass the other three kinematic cuts, with a Gaussian function. We implemented the same algorithm to sample the background in the bins. We fitted the $\Delta \phi$ tail in the range $160^{\circ}-172^{\circ}$ to the left of the peak and $188^{\circ}-200^{\circ}$ to the right of the peak to sample the background at low $Q^{2}$ and low $\varepsilon$ bins. We then subtracted the sampled background from the raw signal to obtain the background subtracted yield. Background for both positive and negative torus polarities were found to be very similar. Even though there was a negligibly small background at high $\varepsilon$ bins, the same algorithm was used to subtract the background to remain consistent in our analysis. 

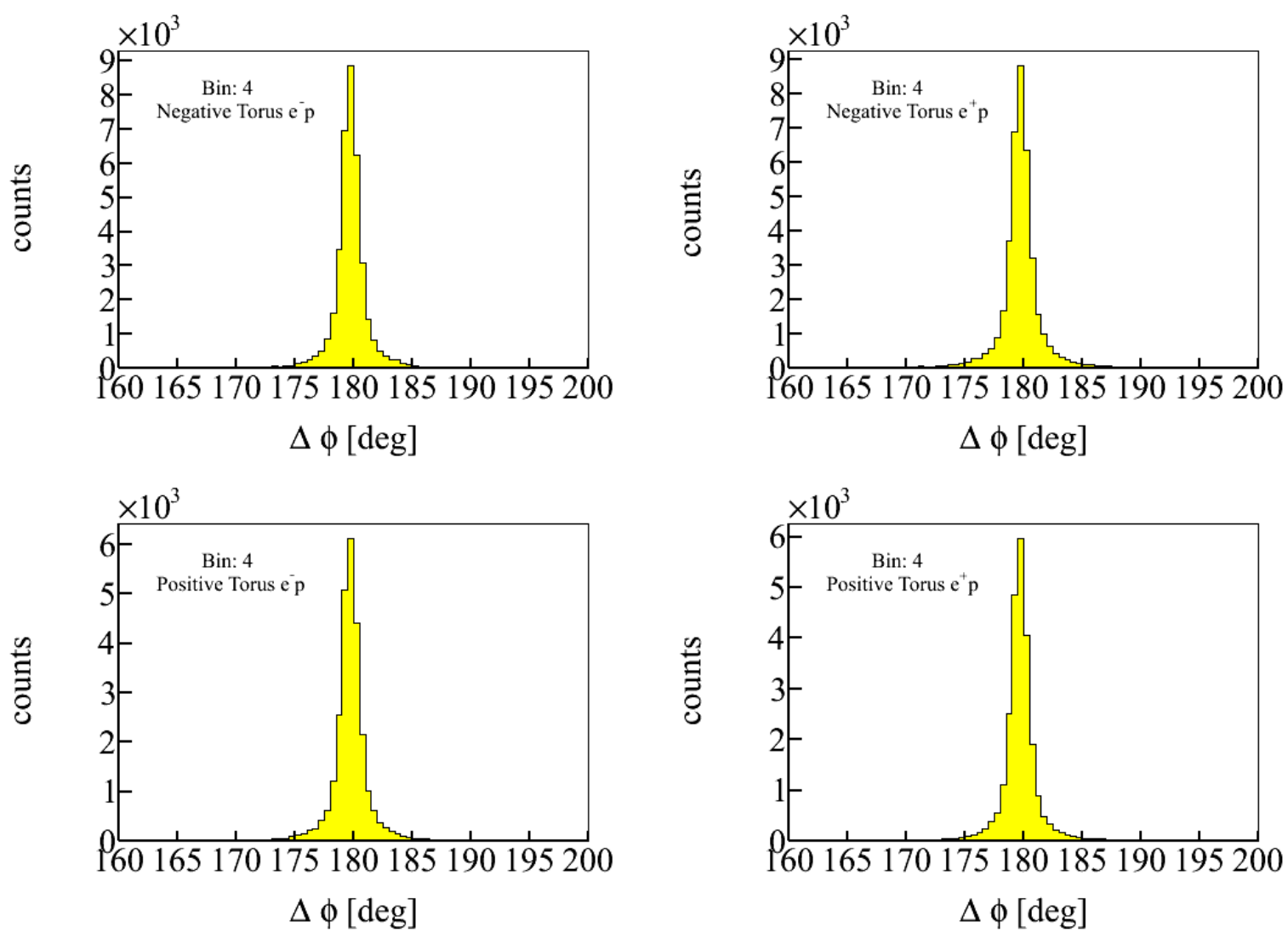

Figure 4.18: $\Delta \phi$ distribution at high $\varepsilon$ and low $Q^{2}$ bin where $\langle\varepsilon\rangle \approx 0.9$ and $\left\langle Q^{2}\right\rangle=0.85$ $\mathrm{GeV}^{2}$ for the events that pass all other cuts. Top left: Negative torus $e^{-} p$ events; Top right: Negative torus $e^{+} p$ events; Bottom left: Positive torus $e^{-} p$ events; Bottom right: Positive torus $e^{+} p$ events. There are no visible tails in these distributions in comparison to the peak, which indicates that the background is negligible

\subsection{The Cross Section Ratio}

The elastic scattering cross section can be measured experimentally by using Eq. 4.11.

$$
\sigma \propto \frac{N}{\mathcal{L} A \delta}
$$

where, $N$ is the number of detected elastic events, $\mathcal{L}$ is the integrated luminosity, $A$ is the detector acceptance and $\delta$ is the radiative correction. To calculate the ratio $R=\frac{\sigma\left(e^{+} p\right)}{\sigma\left(e^{-+} p\right)}$, we must ensure the same detector acceptances for both event types. We first calculate a raw ratio of $e^{+} p$ and $e^{-} p$ elastic events for a given torus and chicane 

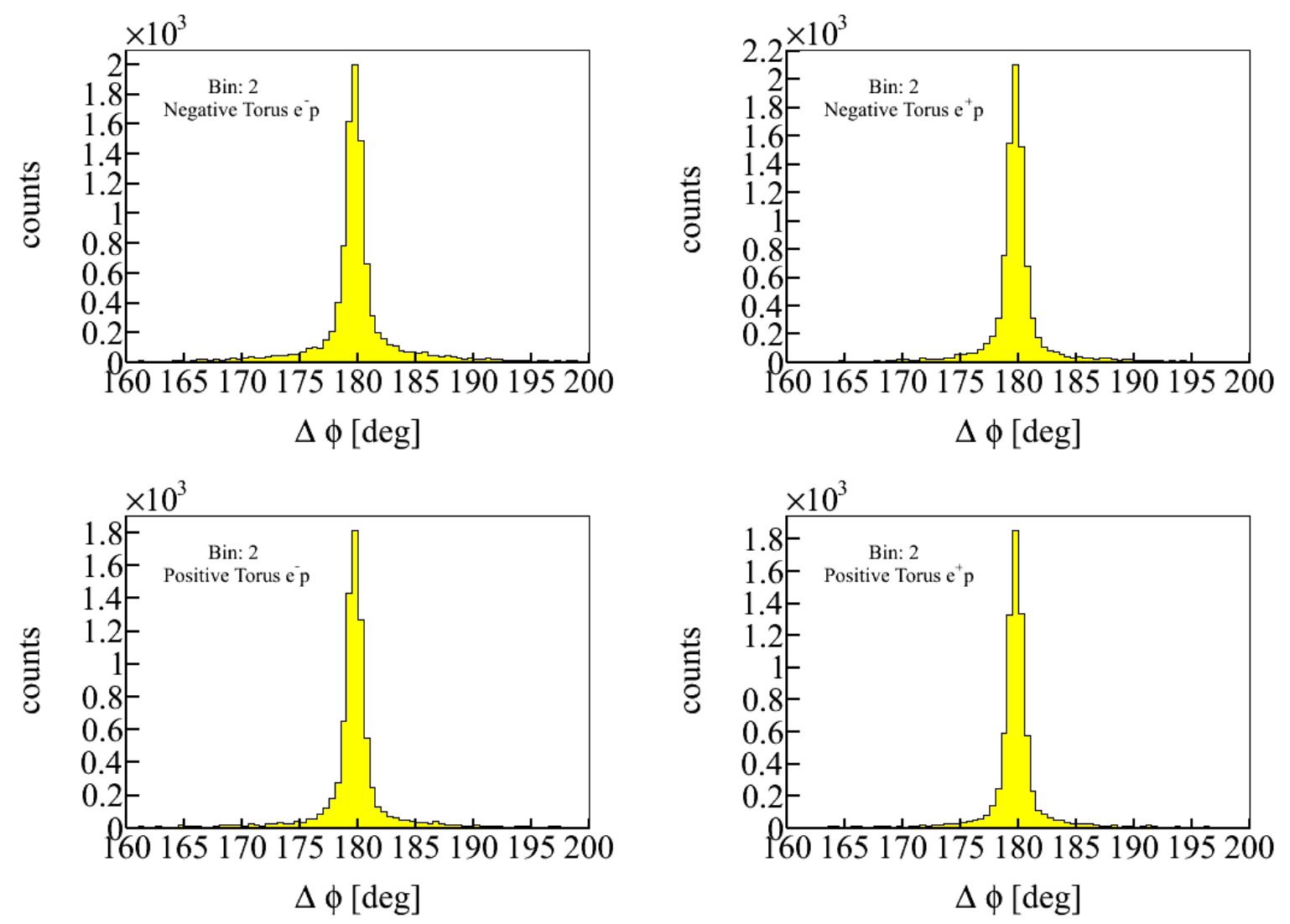

Figure 4.19: $\Delta \phi$ distribution at $\langle\varepsilon\rangle \approx 0.39$ and $\left\langle Q^{2}\right\rangle=0.85 \mathrm{GeV}^{2}$ for the events that pass all other cuts. Top left: Negative torus $e^{-} p$ events; Top right: Negative torus $e^{+} p$ events; Bottom left: Positive torus $e^{-} p$ events; Bottom right: Positive torus $e^{+} p$ events. There are visible tails in these distributions in comparison to the peak, which indicates that the background is not negligible

polarity as,

$$
R_{T}^{C}=\left(\frac{N^{e^{+} p}}{N^{e^{-} p}}\right)_{T}^{C}
$$

and the associated statistical uncertainty,

$$
d R_{T}^{C}=R_{T}^{C} \sqrt{\frac{1}{N^{e^{+} p}}+\frac{1}{N^{e^{-} p}}}
$$



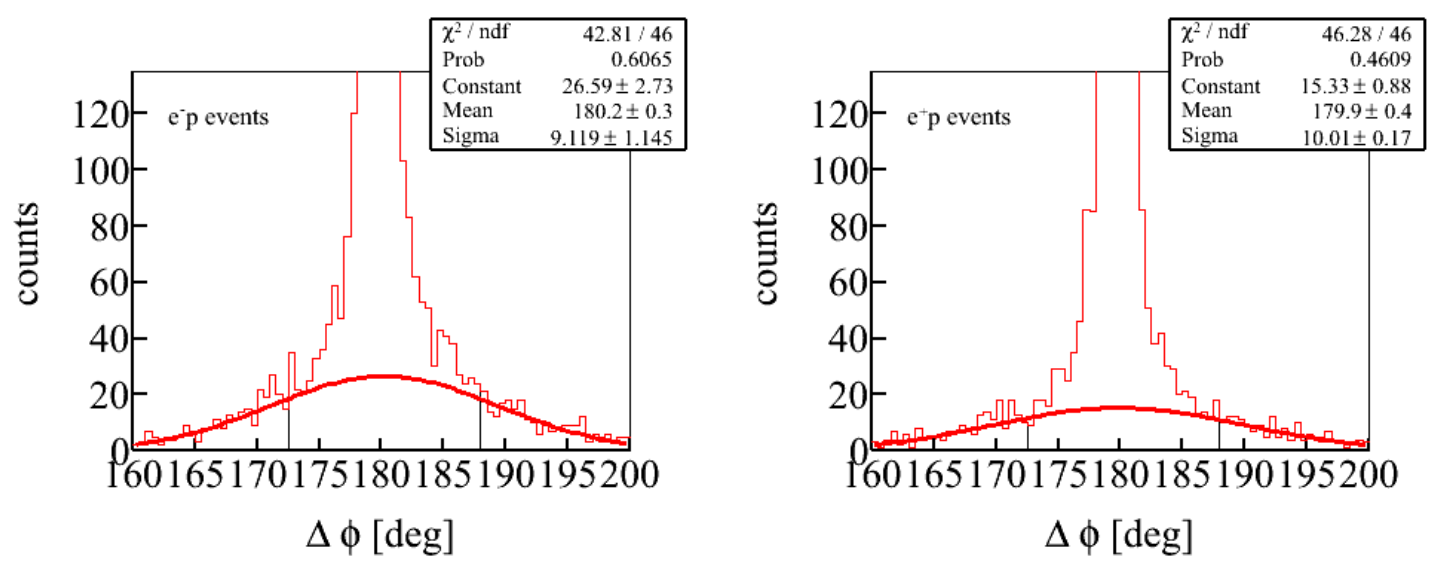

Figure 4.20: Background subtraction for $e^{-} p$ events (left) and $e^{+} p$ events (right). Tails of the $\Delta \phi$ distribution to the left of $172^{\circ}$ and to the right of $188^{\circ}$ (shown by vertical lines) gives the background and was fitted with a Gaussian function. The red curve is the fit to the background.

where the superscript $\mathrm{C}$ indicates the chicane polarity and the subscript $\mathrm{T}$ indicates the torus polarity. The proton acceptances for both event types in the given kinematics in the given torus polarity are the same and cancel in the ratio. However, the acceptances for electron and positron differ as one bends towards the beamline and other bends away from it in the magnetic field. As discussed earlier, we carefully studied the lepton acceptance differences and minimized the difference by applying the fiducial cuts, the dead-detector cuts, and the swimming algorithm. Fig. 4.21 shows the single ratios in different torus and chicane polarities as a function of $\left\langle Q^{2}\right\rangle$ for high $\varepsilon(\varepsilon>0.8)$ bins. To remove any remaining torus-polarity-related acceptance differences, we measured $R_{T}^{C}$ in both torus polarities to form a double ratio $R_{d}^{C}$. For the given chicane polarity, the torus-polarity-independent ratio can be obtained by taking the square root of the product of two single-polarity ratios $R_{+}^{C}$ and $R_{-}^{C}$ for the positive and negative torus polarity [49],

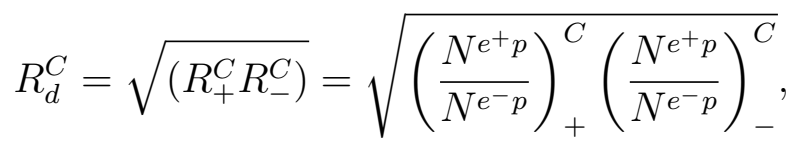



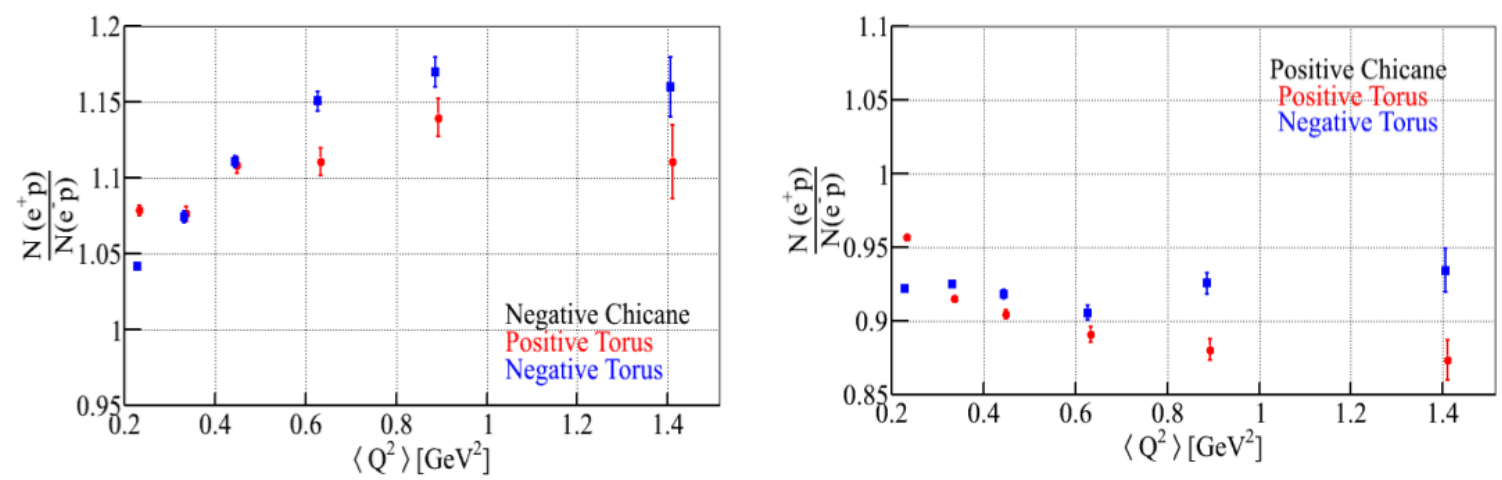

Figure 4.21: The ratio of the number of elastic $e^{+} p$ events to the $e^{-} p$ events for negative chicane polarity (left) and positive chicane polarity (right) as a function of $\left\langle Q^{2}\right\rangle$ at $\langle\varepsilon\rangle \approx 0.88$. The filled (red) circles are the single ratios for the positive torus polarity and the filled squares (blue) are the ratios from the negative torus polarity.

with the associated uncertainty,

$$
d R_{d}^{C}=\frac{R_{d}^{C}}{2} \sqrt{\left(\frac{d R_{+}^{C}}{R_{+}^{C}}\right)^{2}+\left(\frac{d R_{-}^{C}}{R_{-}^{C}}\right)^{2}} .
$$

Any $e^{+}$and $e^{-}$beam-related asymmetries are removed in the quadruple ratio $R_{\text {meas }}$, formed by taking a square root of the product of the torus-double ratios $d R_{d}^{+}$and $d R_{d}^{-}$for the positive and the negative chicane, i.e.,

$$
R_{\text {meas }}=\sqrt{R_{d}^{+} R_{d}^{-}}=\sqrt[4]{R_{+}^{+} R_{-}^{+} R_{+}^{-} R_{-}^{-}}
$$

with the associated uncertainty,

$$
d R_{\text {meas }}=\frac{R_{q}}{2} \sqrt{\left(\frac{d R_{d}^{+}}{R_{d}^{+}}\right)^{2}+\left(\frac{d R_{d}^{-}}{R_{d}^{-}}\right)^{2}}
$$

Fig. 4.22 shows the double ratios in positive and negative chicane polarities, and also 


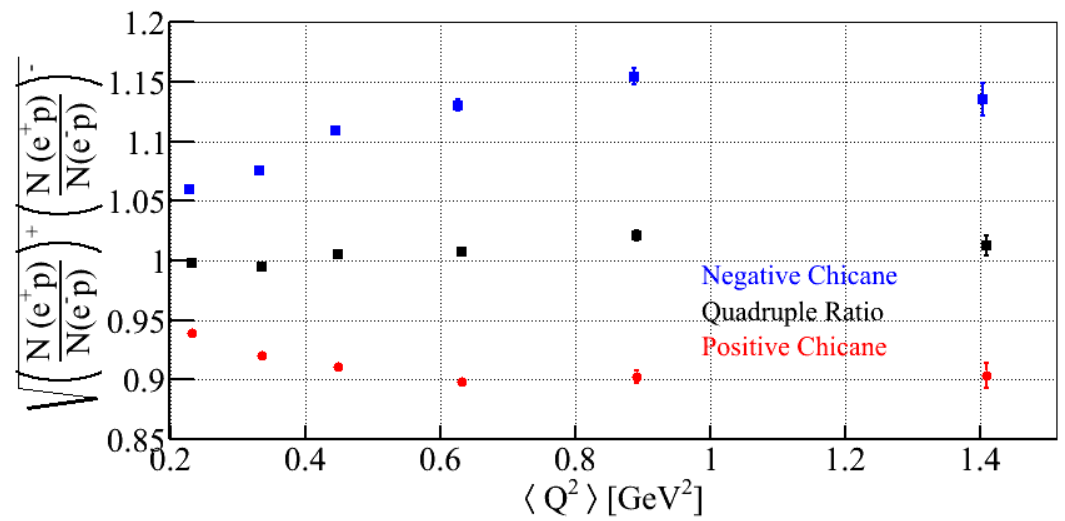

Figure 4.22: Double ratio as a function of $\left\langle Q^{2}\right\rangle$ at $\langle\varepsilon\rangle \approx 0.88$ for negative chicane polarity (blue squares) and positive chicane polarity (red squares). The black squares show the quadruple ratio.

the quadruple ratio as a function of $\left\langle Q^{2}\right\rangle$ for high $\varepsilon(\varepsilon>0.8)$ bins.

\subsection{Results from the Analysis of TPECal Data}

As discussed in Sect. 3.4.4, we took special data using the TPE Calorimeter. The data were used to measure the beam-energy profiles of the individual lepton beams and to measure the extent to which they are identical. To collect TPECal data in a given chicane polarity, one lepton beam was stopped by the lepton blocker and the energy distribution of the other lepton beam was measured in the TPECal and vice versa. We measured the energy distribution of each beam again after reversing the chicane polarity. The collected calorimeter data were analyzed by our collaborators at ODU. Fig. 4.23 shows the the ratio of positron energy to the electron energy as a function of lepton energy when leptons are passing through the left side of the chicane, the same ratio when leptons are passing through the right side of the chicane, and their double ratio given by the square root of their products. It was found that the double ratio of positrons to electrons passing to the left and right side of the chicane was unity and flat, indicating that the left-right asymmetry of the beam cancels in the double ratio [60]. 

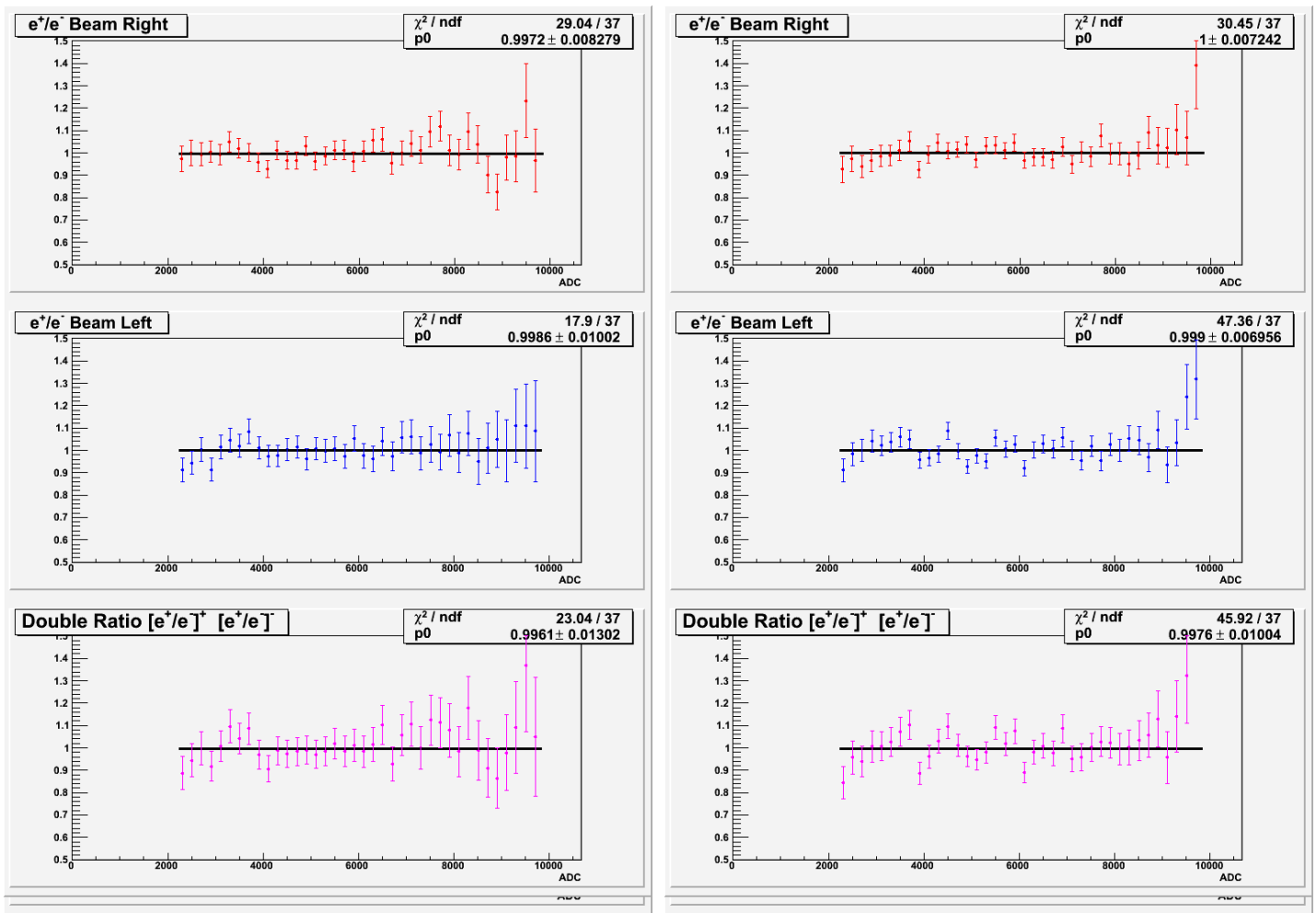

Figure 4.23: The ratio of positron energy to the electron energy as a function of the incident energy (measured in ADC channels where channel 1000 corresponds to an energy of $\approx 370 \mathrm{MeV}$ ). Top row: Leptons passing from the right of the chicane; Middle: leptons passing from the left of the chicane; Bottom: Square root of the product of the two single ratios. The figures on the left panel are when the calorimeter is centered on the beam (nominal position) and the figures on the right are when the calorimeter is offset by $2 \mathrm{~cm}$ from the nominal position. The statistics box shows the parameter of a constant fit to the data. 


\section{Chapter 5}

\section{Results and Discussions}

The main focus of this analysis is to investigate the $Q^{2}$-dependence of the ratio at

high $\varepsilon(\varepsilon>0.8)$ region as well as the $\varepsilon$-dependence of the ratio at low $Q^{2}\left(\left\langle Q^{2}\right\rangle \approx\right.$ $0.85 \mathrm{GeV}^{2}$ ) region. The results from this analysis are presented in this chapter. Table 5.1 summarizes measured quadruple ratios with respective statistical uncertainties. Fig. 5.1 shows the quadruple ratio as a function of $Q^{2}$ at $\langle\varepsilon\rangle \approx 0.88$ and Fig. 5.2 shows the quadruple ratio as a function of $\varepsilon$ at $\left\langle Q^{2}\right\rangle \approx 0.85 \mathrm{GeV}^{2}$. These results have not been corrected for radiative effects. The acceptance related effects due to detector inefficiencies and imperfections largely cancel in the quadruple ratio. Remaining acceptance-related effects lead to a systematic uncertainty that is estimated by studying the CLAS sector-dependence of $R_{\text {meas }}$. Any electron/positron luminosity-related differences lead to additional systematic uncertainties. The process for estimating these and other systematic uncertainties, and for making radiative corrections are discussed in this chapter.

\subsection{Radiative Corrections}

In addition to the leading order Born approximation, the higher order QED radiative processes shown in Fig. 2.6 also contribute to the lepton-nucleon elastic scattering cross section. These contributions, except the TPE diagrams, are usually taken into account as a part of the standard radiative corrections to the measured lepton-nucleon cross section [29, 31]. Some of these radiative corrections are independent of the lepton charge while others depend on it.

It is clear from Eq.2.64 that there are two major lepton-charge dependent corrections to the measured cross section ratio. One is the contribution from the highly model- 
Table 5.1: Measured cross section ratio at high $\varepsilon$ bins before applying radiative corrections

\begin{tabular}{cccccc}
\hline kinematics & Bin & $\left\langle Q^{2}\right\rangle$ & $\langle\varepsilon\rangle$ & $R_{\text {meas }}$ & $\delta R_{\text {stat }}$ \\
\hline \hline \multirow{6}{*}{ High $\varepsilon$} & 1 & 0.232 & 0.915 & 0.995 & 0.0023 \\
& 2 & 0.336 & 0.887 & 0.994 & 0.0026 \\
& 3 & 0.449 & 0.886 & 1.004 & 0.0026 \\
& 4 & 0.632 & 0.885 & 1.013 & 0.0035 \\
& 5 & 0.893 & 0.882 & 1.024 & 0.0046 \\
Low $Q^{2}$ & 6 & 1.415 & 0.874 & 1.015 & 0.0083 \\
\hline \hline & 1 & 0.844 & 0.389 & 1.0268 & 0.0142 \\
& 2 & 0.859 & 0.522 & 1.0057 & 0.0128 \\
& 4 & 0.849 & 0.831 & 1.0226 & 0.0081 \\
& 4 & 0.852 & 0.908 & 1.0074 & 0.0067 \\
\hline
\end{tabular}

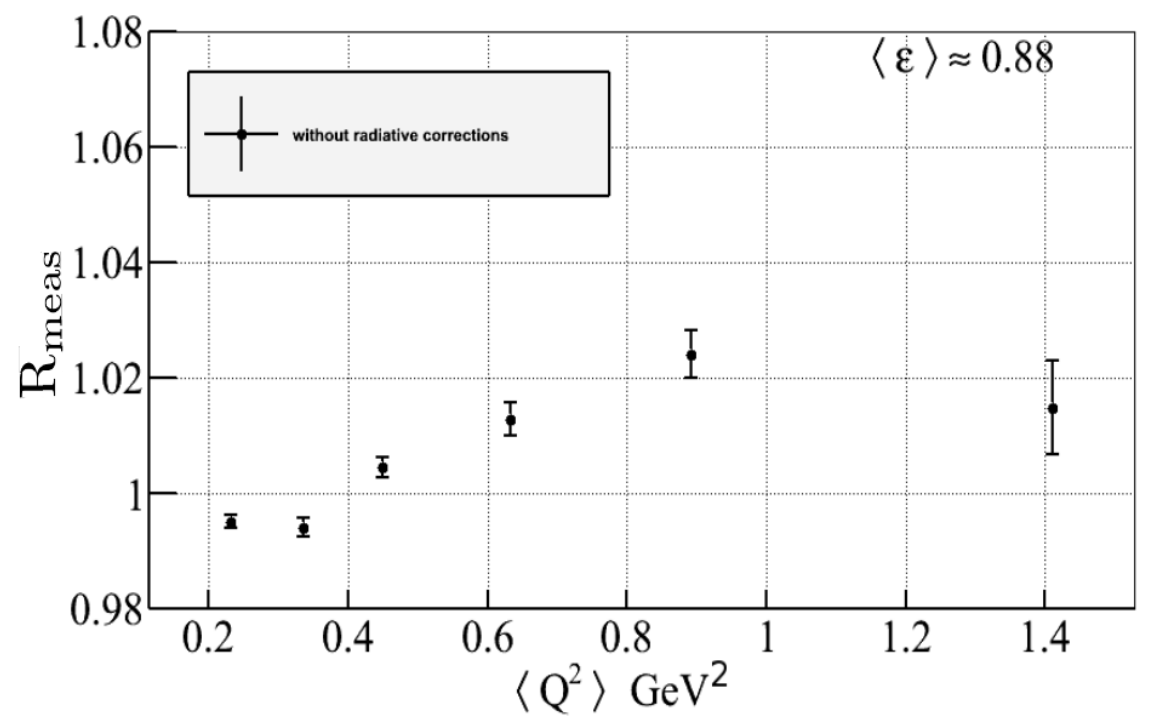

Figure 5.1: Ratio of $e^{+} p$ to $e^{-} p$ yields at high $\varepsilon(\varepsilon>0.8)$, before radiative corrections.

dependent two-photon exchange effects and the other is the contributions from the interference between the electron and proton bremsstrahlung. Our goal is to isolate the two photon exchange effects in a model-independent way. Hence, the measured cross section ratio needs to be corrected for the contribution from the charge-odd electron and proton bremsstrahlung interference, scaled by the charge-even contributions. 


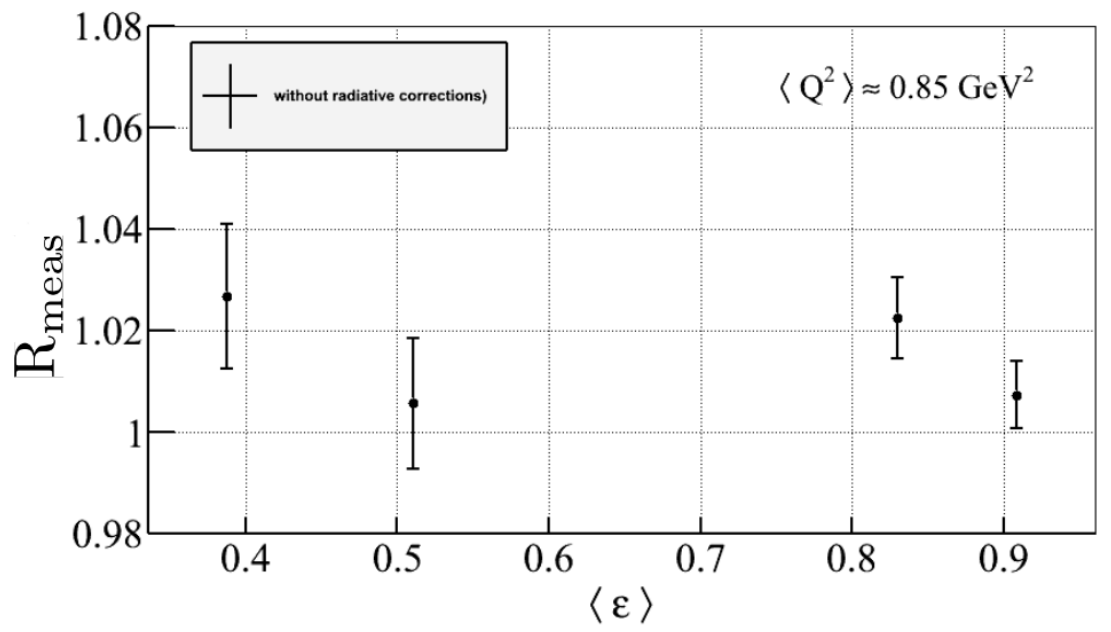

Figure 5.2: Ratio of $e^{+} p$ to $e^{-} p$ yields at low $\left\langle Q^{2}\right\rangle \approx 0.85 \mathrm{GeV}^{2}$, before radiative corrections.

The radiative effects were determined following the general framework given in Ref. [67], taking the 'extended peaking approximation' approach. The radiated photons were generated in the direction of the charged particles and both the incoming and scattered lepton and the struck proton were allowed to radiate. We applied a cut on the effective invariant mass squared $\left(W^{2}\right)$ determined from the our elastic kinematic cuts to select elastic events. Following the formalism given in Ref. [67], the radiative corrections were calculated for $e^{-} p$ scattering. By reversing the sign of the lepton charge in the bremsstrahlung term, we again calculated the corrections. The average of these two corrections yields the charge-even bremsstrahlung contribution $\left(\delta_{\text {even }}\right)$ while the ratio of these two yields the charge-asymmetric contributions, corresponding to the no-TPE limit $R_{2 \gamma}=1-2($ ee.p.br $) /(1+\delta$ even $)$ [60]. We finally obtain the radiative-corrected cross section ratio by dividing the measured cross ratio with the calculated ratio of the radiated $e^{+} p$ cross section to the radiated $e^{-} p$ cross sections. The cross section ratio before and after applying these radiative corrections are shown in Fig. 5.3 and Fig. 5.4 . 


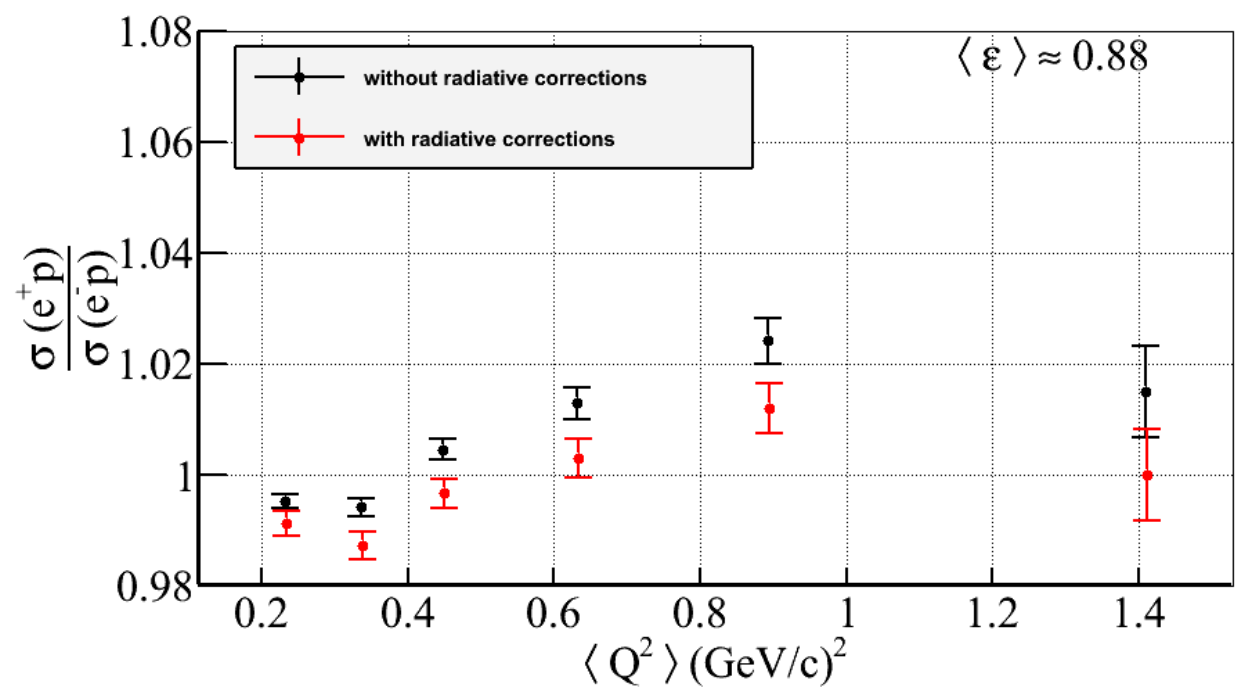

Figure 5.3: Ratio of $e^{+} p$ to $e^{-} p$ elastic scattering cross section as a function of $Q^{2}$. The black points are before radiative corrections and the red points are after applying radiative corrections.

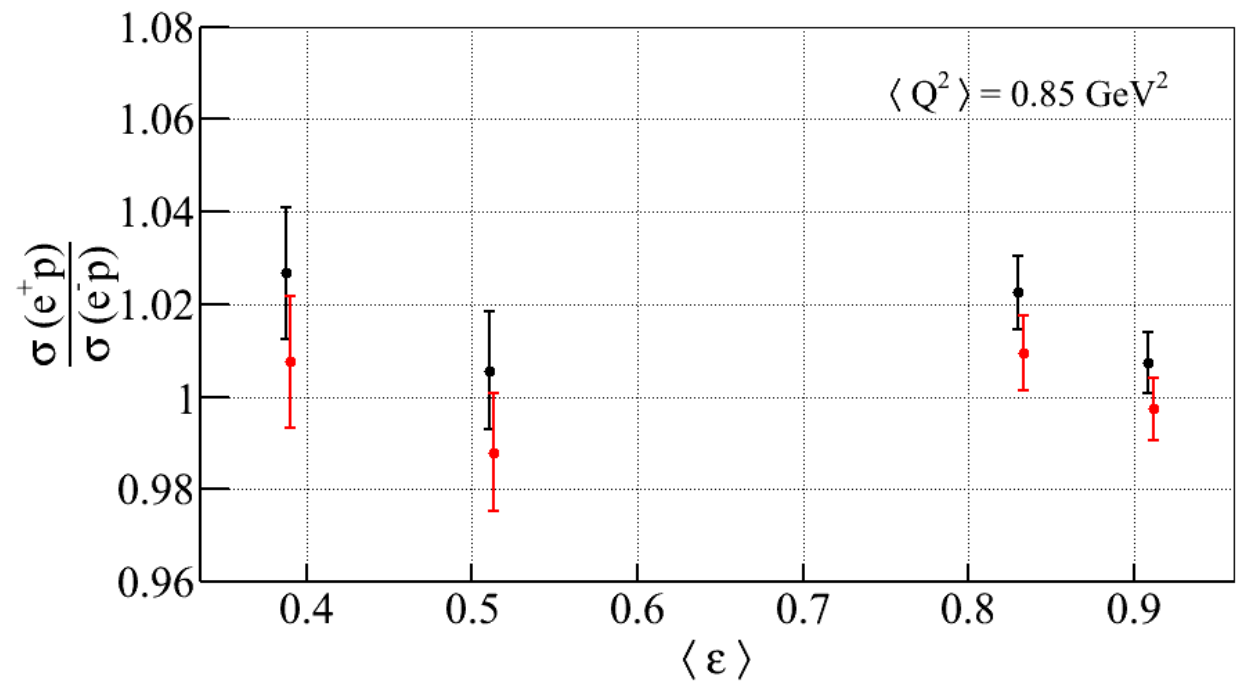

Figure 5.4: Ratio of $e^{+} p$ to $e^{-} p$ elastic scattering cross section as the function of $\varepsilon$. The black points are before radiative corrections and the red points are after applying radiative corrections. 


\subsection{Systematic Uncertainties}

The ratio $R=\frac{\sigma\left(e^{+} p\right)}{\sigma\left(e^{-} p\right)}$ is expected to be within a few percent of unity. Hence, in order to be able to precisely measure the ratio, the systematic uncertainty has to be well under control. To minimize the systematic effects, several strategies were investigated and implemented during planning and commissioning of this experiment. In addition to periodically flipping the torus and chicane magnet polarities to cancel any false asymmetries, TPE calorimeter data were taken before and after each chicane flip to ensure that the luminosity differences between lepton beams are stable and symmetric so that they cancel in the quadruple ratio $\left(R_{\text {meas }}\right)$. Despite our efforts to minimize these effects, some systematic effects still persist, which are required to be estimated and accounted for in the final results. The total systematic uncertainty is estimated by adding contributions from individual sources in a quadrature. We will discuss the most important sources of the systematic uncertainties in this Section.

\subsubsection{Effects of Event Selection Cuts}

The systematic effects due to our event selection cuts were studied in detail. The widths of the event selection cuts were varied from their nominal value. The difference between $R_{\text {meas }}$ with the nominal and the varied cut was estimated as the systematic uncertainty. The following cut related systematic effects were taken into account.

\section{Effect of Fiducial Cuts}

The fiducial cuts that define the good acceptance region for both in-bending and outbending particles were varied from their nominal values by 2 degrees. The difference between $R_{\text {meas }}$ with the nominal and the varied fiducial cut was assigned as the systematic uncertainty associated with the fiducial cut. 


\section{Effect of Vertex Cut}

The effect of the Z-vertex cut on $R_{\text {meas }}$ was studied by reducing the width of the vertex cut from the nominal value $\left(-15 \mathrm{~cm}<V_{z}<-45 \mathrm{~cm}\right)$ by $2 \mathrm{~cm}$ and $3 \mathrm{~cm}$ on each side. The average of the difference between the measured ratio $R_{\text {meas }}$ with the nominal and the varied vertex cuts was estimated to be the systematic uncertainty associated with the vertex cut.

\section{Effect of Elastic Kinematic Cuts}

To study the systematic effects due to the elastic kinematic cuts, the width of each kinematic cut was changed from the nominal $\pm 3.5 \sigma$ to $\pm 3.0 \sigma$ and $\pm 4.0 \sigma$ respectively. The average of the difference between $R_{\text {meas }}$ with the nominal cut and the varied cuts was assigned as the systematic uncertainty due to that cut. The systematic uncertainties due to the four kinematic cuts were then added in a quadrature to obtain the overall systematic uncertainty associated with the elastic kinematic cuts.

\subsubsection{Effect of Ratio Variation with Sectors}

Any remaining CLAS imperfections and detector inefficiencies appear in the variation of the ratio as measured by the six CLAS sectors. Fig. 5.5 shows $R_{\text {meas }}$ as a function of $Q^{2}$ as measured by different CLAS sectors. As mentioned in the previous chapter, events with either a proton or a lepton in the forward region $\left(\theta<45^{\circ}\right)$ of CLAS sector three were removed from the analysis. Removing sector three from the high $\varepsilon$ ( small $\theta$ ) data is equivalent to removing sector six from the low $\varepsilon$ (large $\theta$ ) data since lepton and proton are required to be detected in opposite CLAS sectors (co-planarity). There is a notable variation in the $R_{\text {meas }}$ measured by different sectors, which is used to estimate the acceptance-related systematic uncertainty. We estimated the total 


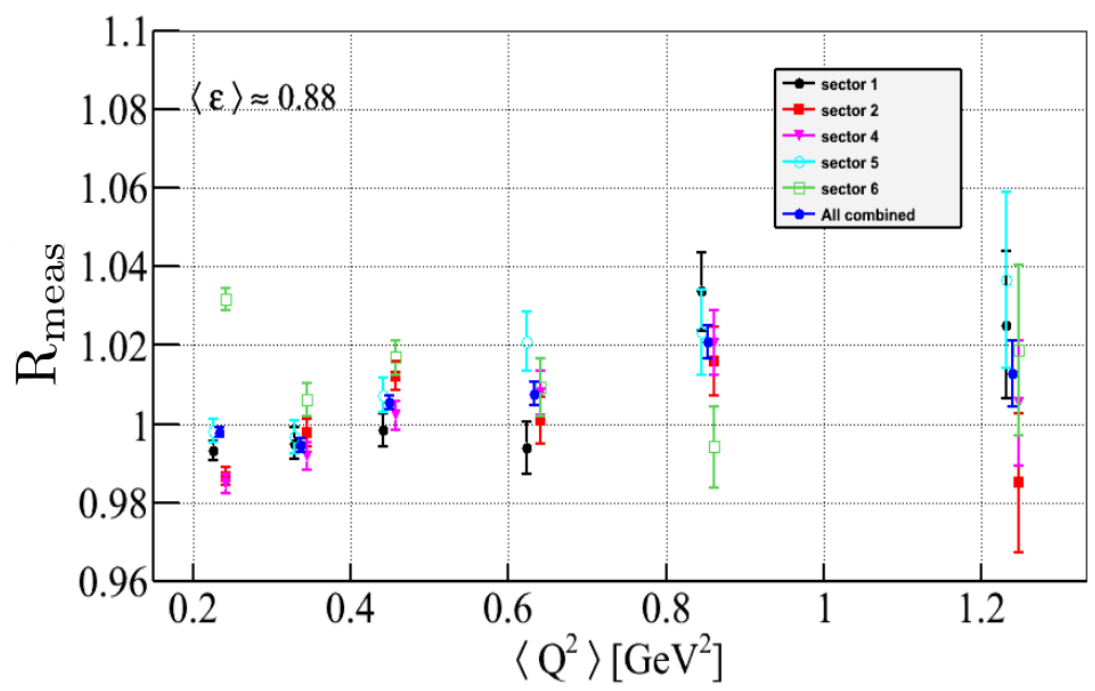

Figure 5.5: Quadruple ratio as a function of $Q^{2}$ as measured by CLAS sectors. The average $\varepsilon$ is 0.88 . The filled circles, filled squares, filled inverted triangles, hollow circles, and hollow squares are the data from CLAS sectors 1, 2, 4, 5, and 6 respectively. The blue filled circles are the combined data from these sectors. The sector 3 data is missing because it was removed from the analysis at high $\varepsilon$.

uncertainty due to the variation in the quadruple ratio measured in different CLAS sectors as:

$$
\delta_{\text {total }}^{2}=\frac{1}{N-1} \sum_{i=1}^{n}\left(\frac{R_{i}-R_{\text {meas }}}{\delta_{i}}\right)^{2}
$$

where $R_{i}$ and $\delta_{i}$ are the quadruple ratio and its statistical uncertainty in each CLAS sector and $N$ is the number of sectors. Eq. 5.1 actually gives a combined statistical and systematic uncertainty. The systematic uncertainty is then obtained as,

$$
\delta_{\text {sys }}=\sqrt{\delta_{\text {total }}^{2}-\delta_{\text {stat }}^{2}}
$$

where $\delta_{\text {stat }}$ is the statistical uncertainty in the overall quadruple ratio $R_{\text {meas }}$. 


\subsubsection{Effect of $e^{+} e^{-}$Luminosity Differences}

This experiment relied on the following pieces of information in order to ensure identical electron and positron luminosity:

- It is known that electron-positron pair production is inherently charge-symmetric.

- It is also known that at energies over $100 \mathrm{MeV}$, which is well below the incident lepton energy threshold of $0.85 \mathrm{GeV}$ for this experiment, the electron and the positron interaction through matter are identical [18] and the difference between Moller and Bhabha scattering cross section is negligible.

- GEANT4 simulations of the beam line show that the electron and positron beams passing on one side of the beamline chicane (e.g., the electron beam passing through the negative chicane and the positron beam passing through the positive chicane) are identical, even though the chicane is not left-right symmetric [59, 61].

- As is clear from the discussion in Sect. 4.6, the flux for $e^{+}$-left is the same as $e^{-}$-left, even though the chicane is not perfectly symmetric. Hence periodically flipping the chicane leads to symmetric luminosities for $e^{+}$and $e^{-}$beams.

As discussed in the previous chapter, any luminosity-related asymmetries must cancel in the measured final ratio. The systematic uncertainties due to any remaining luminosity differences were estimated by measuring the variation in the quadruple ratio with different magnet cycles.

Fig: 5.6 shows $R_{\text {meas }}$ as a function of average $Q^{2}$ for each of the four magnet cycles. It is clear that $R_{\text {meas }}$ for magnet cycle 2 is significantly different from $R_{\text {meas }}$ in the rest of the cycles. In an effort to further investigate this in more detail, the double 


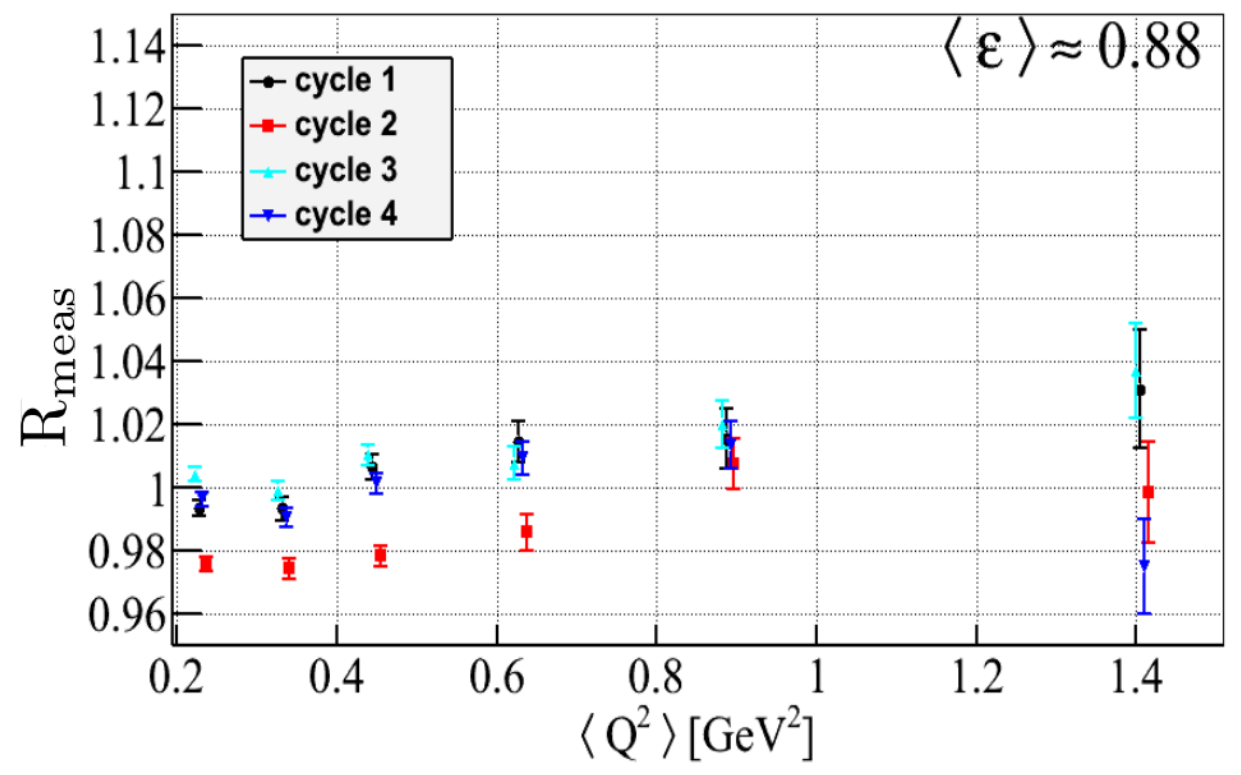

Figure 5.6: Quadruple ratio as a function of $Q^{2}$ for different cycles. The average $\varepsilon$ is 0.88 .

ratios for each chicane polarity for each cycle were also plotted. Fig. 5.7 shows the torus polarity independent double ratios for positive and negative chicane polarity in different magnet cycles. The double ratios for the positive chicane polarity are consistent in all magnet cycles while the double the double ratio for cycle two negative chicane polarity is significantly different from rest of the magnet cycles. These variations were also seen in the cycle dependence of the the calorimeter data [60]. Hence the data from the cycle 2 negative chicane were not used in calculating our final results.

To estimate luminosity-related systematic effects, the systematic uncertainty in the torus-polarity-independent double ratios for each magnet cycle were first calculated. The systematic uncertainty was then propagated to the final measured ratio. For this purpose, double ratios in a given chicane polarity were calculated by taking the square root of the product of the single ratio for positive (negative) torus polarity in a magnet cycle (e.g. cycle 1) with the single ratio for negative (positive) torus polarity 

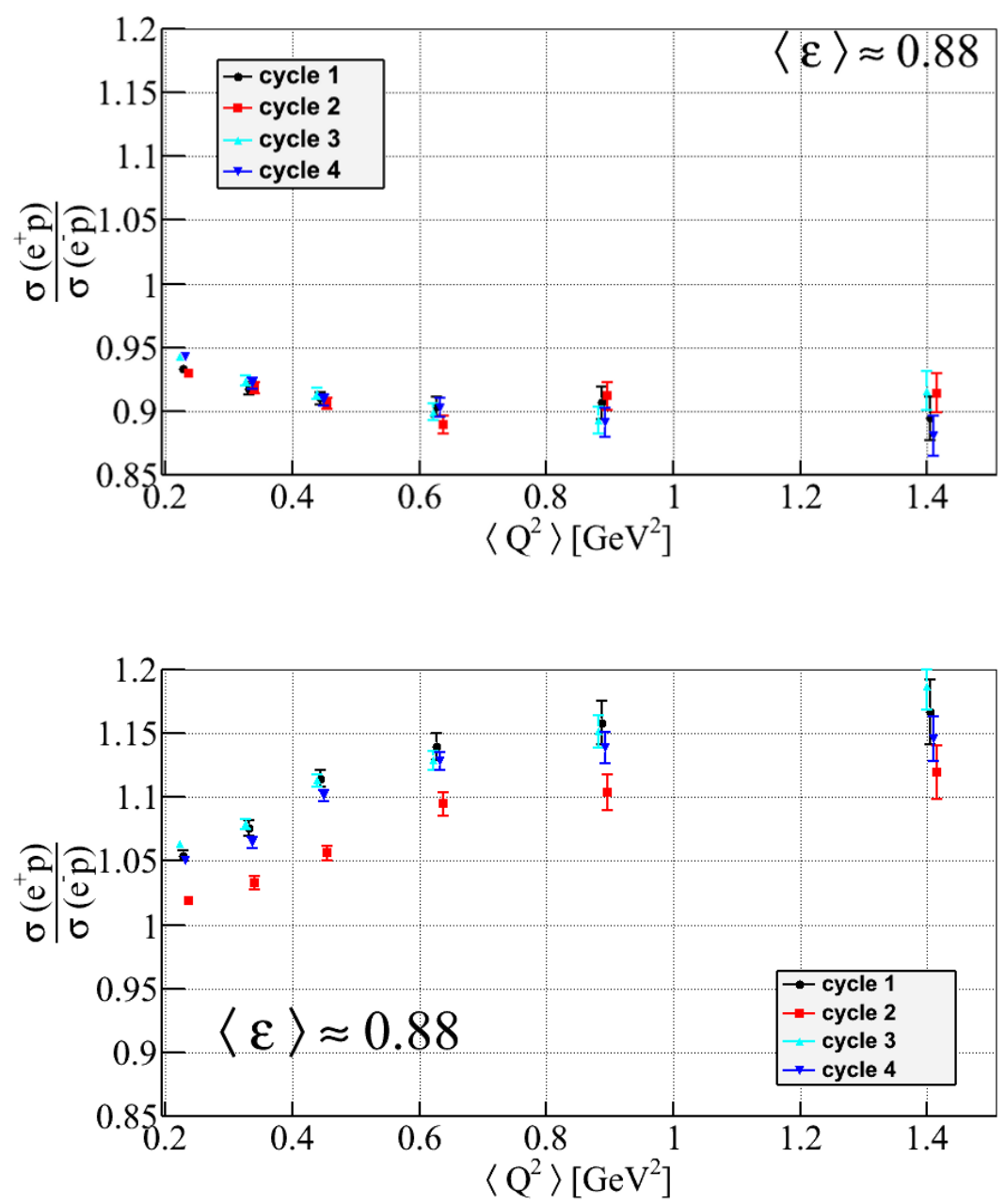

Figure 5.7: Torus polarity independent double ratio as the function of $Q^{2}$ for different magnet cycles for the positive (top) and negative (bottom) chicane polarity

in rest of the magnet cycles (e.g. cycle 2, 3, and 4). A total of 12 possible double ratios were obtained with the data from three complete magnet cycles (cycles 1, 3, and 4) and the positive chicane data from magnet cycle 2. For a given chicane polarity (C), the total uncertainty in the double ratio due to the magnet cycle dependence is given as,

$$
\delta_{\text {total }}^{2}(C)=\frac{1}{N-1} \sum_{i=1}^{n}\left(\frac{R_{i}(C)-R_{\text {overall }}(C)}{\delta_{i}(C)}\right)^{2}
$$


where $R_{i}(C)$ and $\delta_{i}(C)$ are the double ratio and the statistical uncertainty for each magnet cycle in the given chicane setting, $R_{\text {overall }}(C)$ is the double ratio calculated by combining the data from all the magnet cycles in the given chicane setting, and $N$ is the number of complete magnet cycles used. Eq. 5.3 includes both the statistical and systematic uncertainties. Hence, systematic uncertainty can be separated as,

$$
\delta_{\text {sys }}(C)=\sqrt{\delta_{\text {total }}^{2}(C)-\delta_{\text {stat }}^{2}(C)},
$$

where $\delta_{\text {stat }}(C)$ is the statistical uncertainty of the overall double ratio $R_{\text {overall }}(C)$.

Once the systematic uncertainty in each chicane setting is calculated, the systematic uncertainties are then propagated to the quadruple ratio as follows:

$$
\delta_{\text {syst }}=\frac{R_{q}}{2} \sqrt{\left(\frac{\delta_{\text {syst }}(+)}{R(+)}\right)^{2}+\left(\frac{\delta_{\text {syst }}(-)}{R(-)}\right)^{2}},
$$

where the + and the - sign represent the chicane polarity and $R_{q}$ and the $R$ are the quadruple ratio and the double ratio.

\subsubsection{Effect of Background Subtraction}

The systematic effect of the background subtraction was estimated by varying the fitting regions in the tail of the $\Delta \phi$ distribution from the nominal value. As mentioned earlier the nominal range of background fitting was $160^{\circ}-172^{\circ}$ to the left of the peak and $188^{\circ}-200^{\circ}$ to the right of the peak. We calculated $R_{\text {meas }}$ by varying the fitting range by $-2^{\circ}\left(\mathrm{R} 1=160^{\circ}-170^{\circ}: 190^{\circ}: 200^{\circ}\right)$ and by $+2^{\circ}\left(\mathrm{R} 2=160^{\circ}-174^{\circ}: 186^{\circ}-200^{\circ}\right)$. The difference between $R_{\text {meas }}$ with the nominal and the varied fitting ranges were averaged to get the systematic effects due to background subtraction. 
Table 5.2: Estimated systematic uncertainties due to various sources. The - in the $\delta R_{f i d}$ column represents negligible uncertainty.

\begin{tabular}{ccccccccccc}
\hline \hline kinematics & Bin & $\left\langle Q^{2}\right\rangle$ & $\langle\varepsilon\rangle$ & $\delta R_{v z}$ & $\delta R_{\text {kin }}$ & $\delta R_{\text {sector }}$ & $\delta R_{\text {fid }}$ & $\delta R_{\text {bkgsub }}$ & $\delta R_{\text {cycle }}$ & $\delta R_{\text {total }}^{\text {sys }}$ \\
\hline \multirow{4}{*}{ High $\varepsilon$} & 1 & 0.232 & 0.915 & 0.00027 & 0.0012 & 0.0013 & 0.0079 & 0.0028 & 0.0029 & 0.0093 \\
& 2 & 0.336 & 0.887 & 0.00019 & 0.0005 & 0.0006 & 0.0020 & 0.0005 & 0.0022 & 0.0040 \\
& 3 & 0.449 & 0.886 & 0.00023 & 0.0007 & 0.0002 & 0.0028 & 0.0010 & 0.0015 & 0.0043 \\
& 4 & 0.632 & 0.885 & 0.00058 & 0.0011 & 0.0005 & 0.0032 & 0.0052 & 0.000 & 0.0071 \\
& 5 & 0.893 & 0.882 & 0.00084 & 0.0017 & 0.0011 & 0.0046 & 0.0032 & 0.0014 & 0.0077 \\
\hline \hline \multirow{2}{*}{ Low $Q^{2}$} & 6 & 1.415 & 0.874 & 0.00160 & 0.0016 & 0.0041 & 0.0024 & 0.0022 & 0.0092 & 0.0136 \\
\hline \hline & 1 & 0.844 & 0.389 & 0.0075 & 0.00925 & 0.0288 & - & 0.0054 & 0.0168 & 0.0354 \\
& 2 & 0.859 & 0.511 & 0.0112 & 0.00221 & 0.0095 & - & 0.0010 & 0.0149 & 0.0165 \\
& 3 & 0.849 & 0.831 & 0.0027 & 0.00313 & 0.0135 & - & 0.0030 & 0.0078 & 0.0162 \\
& 4 & 0.852 & 0.908 & 0.0005 & 0.00041 & 0.0010 & - & 0.0024 & 0.0010 & 0.0015 \\
\hline \hline
\end{tabular}

\subsubsection{Total Systematic Uncertainties}

The total estimated systematic uncertainty is then obtained by adding the contributions from individual sources in a quadrature i.e.

$$
\delta R_{t o t}=\sqrt{\left(\delta R_{v z}\right)^{2}+\left(\delta R_{\text {kin }}\right)^{2}+\left(\delta R_{\text {fid }}\right)^{2}+\left(\delta R_{\text {sector }}\right)^{2}+\left(\delta R_{\text {bkgsub }}\right)^{2}+\left(\delta R_{\text {cycle }}\right)^{2}}
$$

The estimated systematic errors from different sources are presented in Table 5.2 for different data bins. The total systematic uncertainties for the data at high $\varepsilon$ varies from 0.004 to 0.0136 . Similarly, the total systematic uncertainties for the low $Q^{2}$ data varies from 0.0015 to 0.0354 .

\subsection{Comparison to the World Data and Theoretical Calcula- tions}

Table 5.3 summarizes our final results for the ratio of positron-proton to electronproton elastic scattering cross section along with their associated statistical and systematic uncertainties. In Fig. 5.8, we compare our cross section ratio at high $\varepsilon$ with the currently available world data in a similar $\varepsilon$ range. Our measurements are consistent with the other measurements of the ratio but with a significantly better precision. The blue curve in Fig. 5.8 is the theoretical cross section ratio necessary to resolve the 
Table 5.3: Final cross section ratio and the associated statistical $\left(\delta R_{\text {stat }}\right)$ and systematic uncertainties $\left(\delta R_{\text {sys }}\right.$. The column labeled $R_{\text {meas }}$ is the measured ratio before radiative corrections.

\begin{tabular}{|c|c|c|c|c|c|c|c|}
\hline kinematics & Bin & $\left\langle Q^{2}\right\rangle$ & $\langle\varepsilon\rangle$ & $R_{\text {meas }}$ & $R$ & $\delta R_{\text {stat }}$ & $\delta R_{\text {sys }}$ \\
\hline & 1 & 0.232 & 0.915 & 0.995 & 0.991 & 0.0023 & 0.009 \\
& 2 & 0.336 & 0.887 & 0.994 & 0.987 & 0.0026 & 0.0031 \\
& 3 & 0.449 & 0.886 & 1.004 & 0.997 & 0.0026 & 0.0034 \\
High $\varepsilon$ & 4 & 0.632 & 0.885 & 1.013 & 1.003 & 0.0035 & 0.0062 \\
& 5 & 0.893 & 0.882 & 1.024 & 1.012 & 0.0046 & 0.0062 \\
& 6 & 1.415 & 0.874 & 1.015 & 1.000 & 0.0083 & 0.0108 \\
\hline \multirow{5}{*}{ Low $Q^{2}$} & 1 & 0.844 & 0.389 & 1.0268 & 1.008 & 0.0142 & 0.0354 \\
& 2 & 0.859 & 0.522 & 1.0057 & 0.988 & 0.0128 & 0.0149 \\
& 3 & 0.849 & 0.831 & 1.0226 & 1.009 & 0.0081 & 0.0162 \\
& 4 & 0.852 & 0.908 & 1.0074 & 0.997 & 0.0067 & 0.0015 \\
\hline
\end{tabular}

form factor discrepancy based on the hadronic intermediate state model by Blunden, Melnithouk, and Tjon [36]. Our results indicate a slight $Q^{2}$ dependence of the ratio at high $\varepsilon$. Even though the two-photon exchange contribution is expected to be small at these kinematics, our results appear to confirm the validity of BMT calculations of the ratio.

Fig. 5.9 displays the $\varepsilon$-dependence of our cross section ratio at $\left\langle Q^{2}\right\rangle \approx 0.85 \mathrm{GeV}^{2}$ overlaid with the existing world data at similar $Q^{2}$. The blue curve is the expected ratio from the BMT calculation. Our measurements are consistent with the previous measurements at similar $Q^{2}$ but with a significantly better precision. Our data at these kinematics are still insufficient to make any strong conclusion about the size of the TPE effects. However, our results appear to confirm the validity of BMT calculation at these kinematics.

Fig. 5.10 shows other results from the same experiment at $\left\langle Q^{2}\right\rangle \approx 1.45 \mathrm{GeV}^{2}$. These data were analyzed by another collaborator [66]. Our data are overlaid with the existing world data at similar $Q^{2}[70]$. The blue curve is the expected ratio from the BMT calculation. Our results have a significantly better precision than currently available 


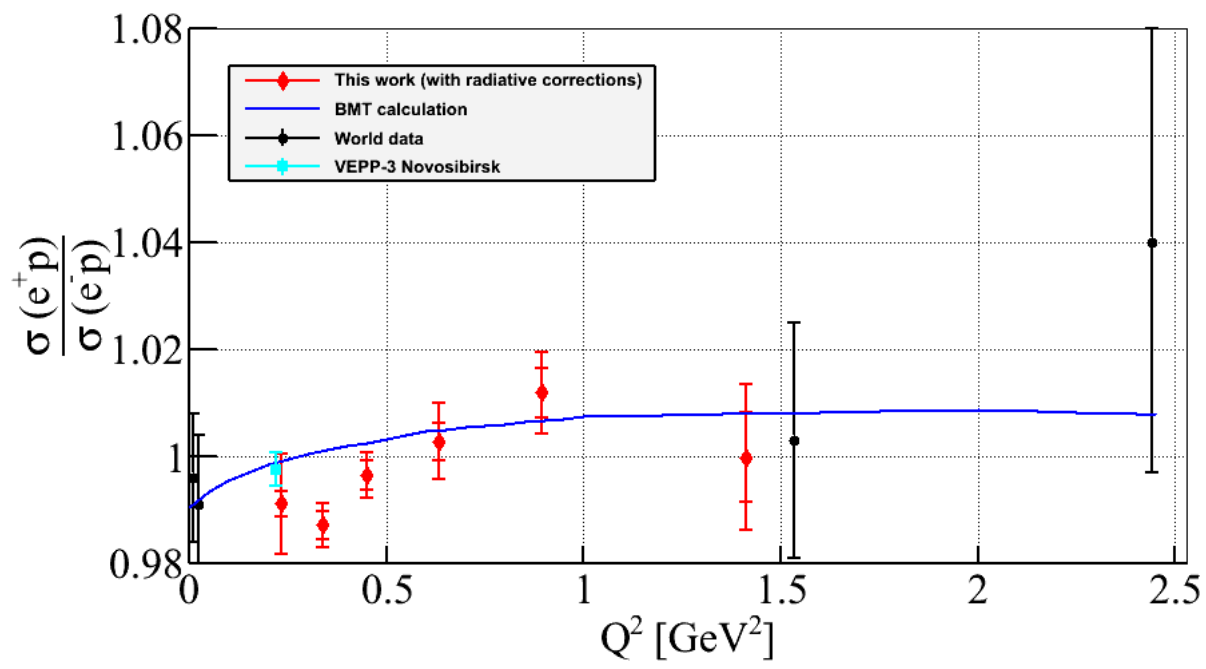

Figure 5.8: $e^{+} p$ to $e^{-} p$ cross section ratio overlaid on the world data. The red diamonds are from this experiment at $\langle\varepsilon\rangle \approx 0.88$, the inner error bars represent statistical uncertainty and the outer error bars represent the total uncertainty. Black filled circles are the world data [68] in a similar $\langle\varepsilon\rangle$ range, the aqua filled square is the preliminary data from VEPP-3 Novosibirsk experiment at $\langle\varepsilon\rangle=0.95$ [69], and the blue curve is the BMT calculation [36] at $\langle\varepsilon\rangle=0.88$ [36].

world data at the similar $Q^{2}$. These results are also consistent with the preliminary Novosibirsk data point at similar kinematics [69] and the BMT calculations.

\subsection{Conclusions and Future Outlook}

The CLAS TPE experiment measured the elastic scattering cross section ratio of $e^{+} p$ to $e^{-} p\left(R=\frac{\sigma\left(e^{+} p\right)}{\sigma\left(e^{-} p\right.}\right)$ over a wide range of kinematics. This dissertation particularly investigated the $Q^{2}$-dependence of $R$ at high $\varepsilon$ as well as the $\varepsilon$ dependence at low $Q^{2}$. The data presented in this dissertation have significantly better precision than currently available world data on $R$ at similar kinematics. All of our results seem to confirm the validity of BMT calculations at the kinematics achieved by the TPE experiment. The BMT calculations, with an additional phenomenological term to include higher excitations of the intermediate nucleon, largely reconciles the Rosenbluth and polarization transfer measurements of the form factor ratio. These measurements 


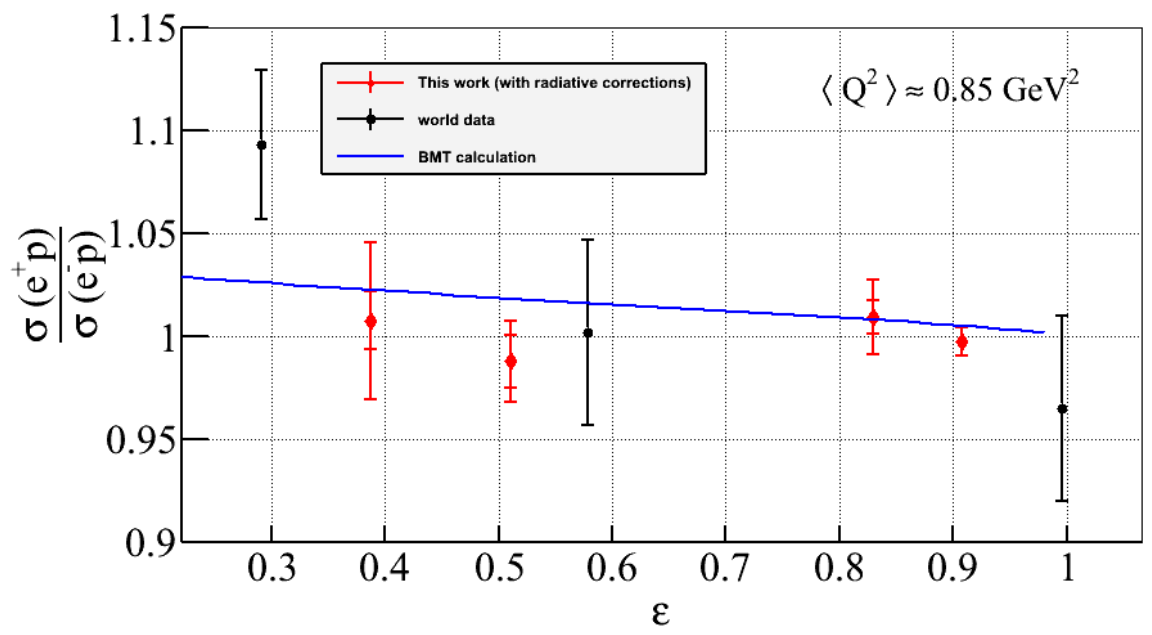

Figure 5.9: $e^{+} p$ to $e^{-} p$ elastic scattering cross section ratio overlaid on the world data. The red diamonds are the data from this experiment at $\left\langle Q^{2}\right\rangle \approx 0.85 \mathrm{GeV}^{2}[70]$. The inner error bars represent statistical uncertainty and the outer error bars represent the total uncertainty. The black filled circles are the world data in a similar $Q^{2}$ range and the blue curve is the BMT calculation at $\left\langle Q^{2}\right\rangle=0.85 \mathrm{GeV}^{2}[36]$.

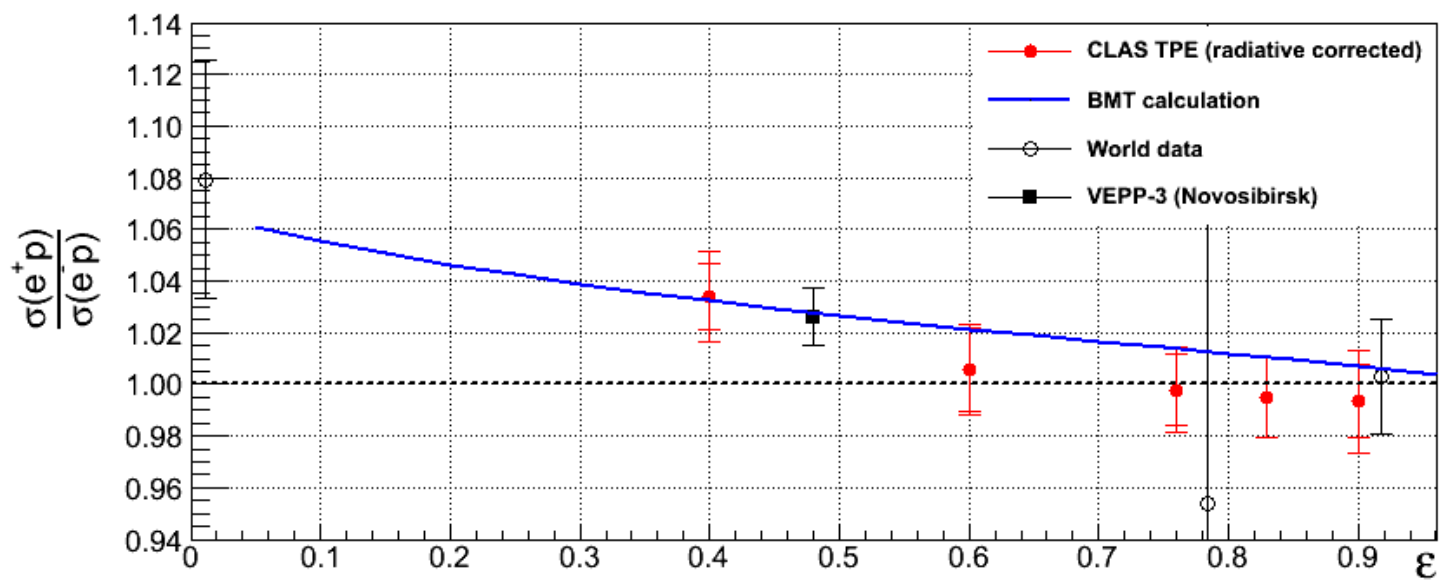

Figure 5.10: $e^{+} p$ to $e^{-} p$ elastic scattering cross section ratio overlaid on the world data. The red filled circles are the data from this experiment at $\left\langle Q^{2}\right\rangle \approx 1.45 \mathrm{GeV}^{2}$. The inner error bars represent statistical uncertainty and the outer error bars represent the total uncertainty. The hollow circles are the world data in a similar $Q^{2}$ range [70], the black filled square is the preliminary Novosibirsk data point, and the blue curve is the BMT calculation at $\left\langle Q^{2}\right\rangle=1.45 \mathrm{GeV}^{2}$ [36]. 
are expected to have a significant impact in placing experimental constraints on the largely diverse theoretical models attempting to explain the form factor discrepancy in terms of the two-photon exchange effects.

Currently, two other experiments are also analyzing data to measure the ratio $R$ to determine the TPE effect. Some preliminary data points from the Novosibirsk group [69] are available, and are consistent with our measurements as discussed earlier. The OLYMPUS Collaboration completed data taking in 2012 at a fixed lepton beam energy corresponding to $Q^{2}<2.5 \mathrm{GeV}^{2}$ [51]. All of these experiments measure the cross section ratio at $Q^{2}<2.5 \mathrm{GeV}^{2}$, where the discrepancy between the Rosenbluth and polarization transfer method is small. The precise measurement of the cross section ratio at significantly higher $Q^{2}$ are necessary to completely resolve the proton form factor puzzle.

Even though the proton has been studied for the past several decades via elastic electron scattering, the discrepancy between the Rosenbluth and polarization transfer measurements of the electromagnetic form factors of the proton revealed our incomplete understanding of the internal structure of proton including the charge and magnetization distribution. The results from the CLAS TPE experiment along with the results from other two experiments will provide significant information that is crucial in understanding the elastic electron-nucleon scattering process and the internal structure of the nucleon. 


\section{Bibliography}

[1] O. Stern et al., "Magnetic moment of the proton," Nature 132, 169-170 (1933).

[2] R. Hofstadter, H. R. Fechter, and J. A. McIntyre, "High-energy electron scattering and nuclear structure determinations," Phys. Rev. 92, 978-987 (1953).

[3] J. Arrington, C. D. Roberts, and J. M. Zanotti, "Nucleon electromagnetic form factors," J. Phys. G 34, 23 (2007).

[4] C. F. Perdrisat, V. Punjabi, and M. Vanderhaeghen, "Nucleon electromagnetic form factors," Prog. Part. Nucl. Phys. 59, 694 (2007).

[5] John Arrington, Kees de Jager, and Charles F. Perdrisat, "Nucleon Form Factors: A Jefferson Lab Perspective," J. Phys. Conf. Ser. 299, 012002 (2011).

[6] R. C. Walker et al., "Measurement of the proton elastic form factors for $Q^{2}=$ 1-3 (GeV/c) $)^{2}$, Phys. Lett. B 224, $353-358$ (1989).

[7] J. Arrington, "How well do we know the electromagnetic form factors of the proton," Phys. Rev. C 68, 034325 (2003).

[8] M. N. Rosenbluth, "High-energy elastic scattering of electrons on protons," Phys. Rev. 79, 615 (1950).

[9] L. Andivahis et al., "Measurements of the electric and magnetic form-factors of the proton from $Q^{2}=1.75-(\mathrm{GeV} / \mathrm{c})^{2}$ to $8.83-(\mathrm{GeV} / \mathrm{c})^{2}$," Phys. Rev. D. 50, 5491-5517 (1994).

[10] M. E. Christy et al., "Measurements of electron proton elastic cross sections for $0.4-(\mathrm{GeV} / \mathrm{c})^{2}<Q^{2}<5.5-(\mathrm{GeV} / \mathrm{c})^{2}$," Phys. Rev. C 70, 015206 (2004).

[11] I. A. Qattan et al., "Precision rosenbluth measurement of the proton elastic form factors," Phys. Rev. Lett. 94, 142301 (2005).

[12] M. K. Jones et al., " $\frac{G_{E p}}{G_{M p}}$ ratio by polarization transfer in $\overrightarrow{e p} \rightarrow e \vec{p}$," Phys. Rev. Lett. 84, 1398-1402 (2000).

[13] O. Gayou et al., "Measurements of the elastic electromagnetic form factor ratio $\mu_{p} G_{E_{p}} / G_{M_{p}}$ via polarization transfer," Phys. Rev. C 64, 038202 (2001).

[14] O. Gayou et al., "Measurement of $G_{E_{p}} / G_{M_{p}}$ in $\overrightarrow{e p} \rightarrow e \vec{p}$ to $Q^{2}=5.6(\mathrm{GeV} / \mathrm{c})^{2}$," Phys. Rev. Lett. 88, 092301 (2002).

[15] V. Punjabi et al., "Proton elastic form factor ratios to $Q^{2}=3.5(\mathrm{GeV} / \mathrm{c})^{2}$ by polarization transfer," Phys. Rev. C 71, 055202 (2005). 
[16] P. G. Blunden, W. Melnitchouk, and J. A. Tjon, "Two-photon exchange and elastic electron-nucleon scattering," Phys. Rev. C 72, 034612 (2005).

[17] M. E. Peskin and D. V. Schroeder, An Introduction to Quantum Field Theory (Addison-Wesley, Reading, Massachusetts, 1995).

[18] J. Beringer et al. (Particle Data Group), "Review of particle physics," Phys. Rev. D 86, 010001 (2012).

[19] D. Yennie, M. Levy, and D. Ravenhall, Rev. Mod. Phys. 29, 144 (1957).

[20] R. Sachs F. Ernst and K. Wali, Phys. Rev. 119, 1105 (1960).

[21] J.C. Bernauer et al., "High-precision determination of the electric and magnetic form factors of the proton," Phys. Rev. Lett. 105, 242001 (2010).

[22] R. Pohl et al., "The size the proton," Nature 466, 1589 (2010).

[23] P. J. Mohr, B. N.Taylor, and D. B. Newell, "CODATA recommended values of the fundamental physical constants: 2010," Rev. Mod. Phys. 84, 213-216 (2010).

[24] Peter G. Blunden and Ingo Sick, "Proton radii and two-photon exchange," Phys. Rev. C 72, 057601 (2005).

[25] Issam A. Qattan, Precision Rosenbluth Measurement of the Proton Elastic Electromagnetic Form Factors and Their Ratio at $Q^{2}=2.64$, 3.20, and $4.10 \mathrm{GeV}^{2}$, Ph.D. thesis, Northwestern University (2005).

[26] Raymond G. Arnold, Carl E. Carlson, and Franz Gross, "Polarization transfer in elastic electron scattering from nucleons and deuterons," Phys. Rev. C 23, 363-374 (1981).

[27] G. Ron et al., "Measurements of the proton elastic-form-factor ratio $G_{E_{p}} / G_{M_{p}}$ at low momentum transfer," Phys. Rev. Lett. 99, 202002 (2007).

[28] G. MacLachlan et al., "The ratio of proton electromagnetic form factors via recoil polarimetry at $Q^{2}=1.13(\mathrm{GeV} / \mathrm{c})^{2}$," Nucl. Phys. A 764, $261(2006)$.

[29] Luke W. Mo and Yun-Su Tsai, "Radiative corrections to elastic and inelastic ep and $\mu p$ scattering," Rev. Mod. Phys. 41, 205-235 (1969).

[30] J. Arrington, P.G. Blunden, and W. Melnitchouk, "Review of two-photon exchange in electron scattering," Prog. Part. Nucl. Phys. 66, 782-833 (2011).

[31] L. C. Maximon and J. A. Tjon, "Radiative corrections to electron proton scattering," Phys. Rev. C 62, 054320 (2000). 
[32] P. A. M. Guichon and M. Vanderhaeghen, "How to reconcile the rosenbluth and the polarization transfer method in the measurement of the proton form factors," Phys. Rev. Lett. 91, 142303 (2003).

[33] Andrei V. Afanasev, Stanley J. Brodsky, Carl E. Carlson, Yu-Chun Chen, and Marc Vanderhaeghen, "Two-photon exchange contribution to elastic electronnucleon scattering at large momentum transfer," Phys. Rev. D 72, 013008 (2005).

[34] S. D. Drell and S. Fubini, "Higher electromagnetic corrections to electron-proton scattering," Phys. Rev. 113, 741 (1959).

[35] Gary K. Greenhut, "Two-photon exchange in electron-proton scattering," Phys. Rev. 184, 1860 (1969).

[36] P. G. Blunden, W. Melnitchouk, and J. A. Tjon, "Two-photon exchange in elastic electron nucleon scattering," Phys. Rev. C 72, 034612 (2005).

[37] P. G. Blunden, W. Melnitchouk, and J. A. Tjon, "Two-photon exchange and elastic electron proton scattering," Phys. Rev. Lett. 91, 142304 (2003).

[38] S. Kondratyuk, P. G. Blunden, W. Melnitchouk, and J. A. Tjon, "Delta resonance contribution to two-photon exchange in electron proton scattering," Phys. Rev. Lett. 95, 172503 (2005).

[39] S. Kondratyuk and P. G. Blunden, "Contribution of spin 1/2 and 3/2 resonances to two-photon exchange effects in elastic electron-proton scattering," Phys. Rev. C 75, 038201 (2007).

[40] Dmitry Borisyuk and Alexander Kobushkin, "Box diagram in the elastic electronproton scattering," Phys. Rev. C 74, 065203 (2006).

[41] Yu-Chun Chen, Chung-Wen Kao, and Shin-Nan Yang, "Is there model independent evidence of the two photon exchange effect in the electron-proton elastic scattering cross-section," Phys. Lett. B 652, 269-274 (2007).

[42] Dmitry Borisyuk and Alexander Kobushkin, "Two-photon exchange at low $Q^{2}$," Phys. Rev C75, 038202 (2007).

[43] Dmitry Borisyuk and Alexander Kobushkin, "Phenomenological analysis of twophoton exchange effects in proton form-factor measurements," Phys. Rev. C 76, 022201 (2007).

[44] Mikhail Gorchtein, "Dispersive contributions to cross section ratio in forward regime," Physics Letters B 644, 322 - 330 (2007).

[45] Nikolai Kivel and Marc Vanderhaeghen, "Two-photon exchange in elastic electron-proton scattering: QCD factorization approach," Phys. Rev. Lett. 103, 092004 (2009). 
[46] M.P. Rekalo and E. Tomasi-Gustafsson, "Polarization phenomena in elastic scattering, for axial parametrization of two-photon exchange," Nuclear Physics A $\mathbf{7 4 2}, 322-334$ (2004).

[47] Carl E. Carlson and Marc Vanderhaeghen, "Two-photon physics in hadronic processes," Ann. Rev. Nucl. Part. Sci. 57, 171 (2007).

[48] Y. C. Chen, A. Afanasev, S. J. Brodsky, C. E. Carlson, and M. Vanderhaeghen, "Partonic calculation of the two-photon exchange contribution to elastic electron proton scattering at large momentum transfer," Phys. Rev. Lett. 93, 122301 (2004).

[49] M. Moteabbed et al. (CLAS Collaboration), "Demonstration of a novel technique to measure two-photon exchange effects in elastic $e^{ \pm} p$ scattering," Phys. Rev. C 88, 025210 (2013).

[50] J. Arrington et al., "Beyond the born approximation: A precise comparison of $e^{+} p$ and $e^{-} p$ elastic scattering in clas," Jefferson Lab Experiment E07-005(2007).

[51] The Proposal and Technical Design Report for the OLYMPUS experiment can be found at http://web.mit.edu/OLYMPUS.

[52] J. Arrington et al., "Two-photon exchange and elastic scattering of electrons/positrons on the proton. (Proposal for an experiment at VEPP-3)," (2004), arxiv:0408020 [nucl-ex].

[53] C. W. Leemann, D. R. Douglas, and G.A. Krafft, "The Continuous Electron Beam Accelerator Facility:CEBAF at the Jefferson Laboratory," Annu. Rev. Nucl. Part. Sci. 51, 413 (2001).

[54] "12 GeV updates," http://www.jlab.org/12GeV/updates.html (2013).

[55] B. Mecking et al., "The CEBAF Large Acceptance Spectrometer(CLAS)," Nucl. Inst. Meth. A 503, 513 (2003).

[56] M.D Mestayer et al., "The CLAS drift chamber system," Nucl. Inst. Meth. 449, $81-111(2000)$.

[57] E.S. Smith et al., "The time-of-flight system for CLAS," Nucl. Inst. Meth. 432, $265-298$ (1999).

[58] M. Amarian et al., "The CLAS forward electromagnetic calorimeter," Nucl. Inst. Meth. 460, 239 - 265 (2001).

[59] Maryam Moteabbed, A Precise Measurement of two-photon exchange effect, Ph.D. thesis, Florida International University (2009). 
[60] D. Rimal, D. Adikaram, et al., "Analysis note: The two photon exchange experiment," CLAS-analysis-note (2014).

[61] Megh Niroula, Beyond the Born Approximation: A precise comparison of $e^{+} p$ and $e^{-} p$ elastic scattering in the CLAS, Ph.D. thesis, Old Dominian University (2010).

[62] "Root: An object oriented data analysis framework," https://http://root. cern.ch/drupal/.

[63] E. Pasyuk, "Energy loss corrections for charged particles in CLAS," CLASNOTE-016 (2007).

[64] A. Klimenko and Kuhn S., "Momentum Corrections for E6," CLAS Note 005 (2003).

[65] "g13 fiducial cuts," https://clasweb.jlab.org/rungroups/g13/wiki_ secure/index.php/FiducialCuts.

[66] Dasuni Adikaram, Ph.D. thesis, Old Dominian University (2014).

[67] R. Ent et al., "Radiative corrections for $\left(e, e^{\prime} p\right)$ reactions at Gev energies," Phys. Rev. C 64, 054610 (2001).

[68] J. Mar et al., "A comparison of electron - proton and positron - proton elastic scattering at four momentum transfers up to 5.0-(gev/c) $)^{2}, "$ Phys. Rev. Lett. 21, 482 (1968).

[69] A.V. Gramolin et al., "Measurement of the two-photon exchange contribution in elastic ep scattering at vepp3," in Nucl. Phys. Proc. Suppl., Vol. 225227 (2012) p. 216.

[70] A. Browman, F. Liu, and C. Schaerf, "Positron-proton scattering," Phys. Rev. 139, B1079-1085 (1965). 
VITA

\section{DIPAK RIMAL}

Born, Jhapa, Nepal

$2007-2014$

PhD Candidate

Florida International University

Miami, Florida

Graduate Research Assistant

$2010-2014$

$2007-2009$

Florida International University

Miami, Florida

Graduate Teaching Assistant

Florida International University

Miami, Florida

Faculty Member

$2005-2007$

Xavier Academy College

Kathmandu, Nepal

M.Sc. in Physics

2005

Tribhuvan University

Kathmandu, Nepal

2002

Tribhuvan University Scholarship Award

B.Sc. in Physics

2001

Tribhuvan University

Kathmandu, Nepal

\section{PUBLICATIONS AND PRESENTATIONS}

- D. Rimal, B. Raue et al. Analysis Note: The two-photon exchange experiment, Submitted to the CLAS Collaboration for internal review (2013).

- M. Moteabbed, B. Raue, D. Rimal et al. (CLAS Collaboration), Demonstration of a novel technique to measure two-photon exchange effects in elastic $e^{ \pm} p$ scattering, Phys. Rev. C88, 025210 (2013).

- M. Moteabbed, B. Raue, D. Rimal et al. (CLAS Collaboration), Measurement of Two Photon Exchange Effects Using $e^{ \pm} p$ Elastic Scattering in CLAS. CLAS Analysis-Note (2009).

- D. Rimal et al. $Q^{2}$-dependence of Two-Photon Exchange Effects at $\varepsilon=0.884$. Bulletin of the APS April Meeting, Newport News, VA, 2013. 
- D. Rimal et al. Proton Form Factor Puzzle and the CLAS Two Photon Exchange Experiment - Photonuclear Gordon Research Conference (Poster), Holderness, NH, August 2012 (poster).

- D. Rimal et al. CLAS Two Photon Experiment : Analysis Methods. Bulletin of the APS April Meeting, Anaheim, CA, 2011. 